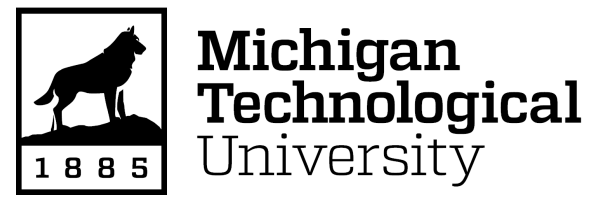

Michigan Technological University Digital Commons @ Michigan Tech

Dissertations, Master's Theses and Master's Reports

2021

\title{
CHINESE WOMEN'S REPRODUCTIVE JUSTICE AND DIGITAL TECHNOLOGIES
}

Hua Wang

Michigan Technological University, huaw@mtu.edu

Copyright 2021 Hua Wang

\section{Recommended Citation}

Wang, Hua, "CHINESE WOMEN'S REPRODUCTIVE JUSTICE AND DIGITAL TECHNOLOGIES", Open Access Dissertation, Michigan Technological University, 2021.

https://doi.org/10.37099/mtu.dc.etdr/1280

Follow this and additional works at: https://digitalcommons.mtu.edu/etdr

Part of the Other Feminist, Gender, and Sexuality Studies Commons, Rhetoric Commons, and the Technical and Professional Writing Commons 
By

Hua Wang

\begin{abstract}
A DISSERTATION
Submitted in partial fulfillment of the requirements for the degree of DOCTOR OF PHILOSOPHY

In Rhetoric, Theory and Culture
\end{abstract}

MICHIGAN TECHNOLOGICAL UNIVERSITY

2021

(C) 2021 Hua Wang 
This dissertation has been approved in partial fulfillment of the requirements for the Degree of DOCTOR OF PHILOSOPHY in Rhetoric, Theory and Culture.

Department of Humanities

$\begin{array}{ll}\text { Dissertation Advisor: } & \text { Dr. Marika Seigel } \\ \text { Committee Member: } & \text { Dr. Robert Johnson } \\ \text { Committee Member: } & \text { Dr. Sarah Bell } \\ \text { Committee Member: } & \text { Dr. Kim Hensley Owens }\end{array}$

Department Chair: $\quad$ Dr. Scott Marratto 


\section{Table of Contents}

Preface

Acknowledgments................................................................................................... vi

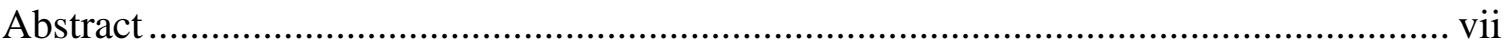

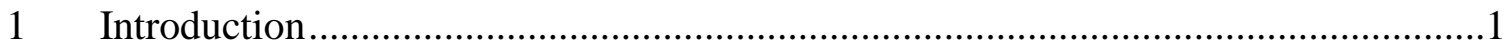

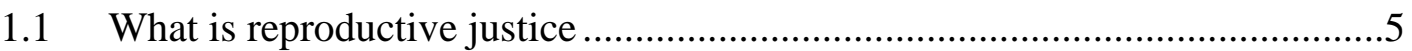

1.2 Reproductive injustice and constrained agency in China................................6

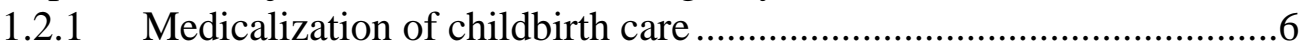

1.2.2 Chinese culture shaping reproductive behavior ..................................

1.2.3 Gender discrimination in employment..............................................12

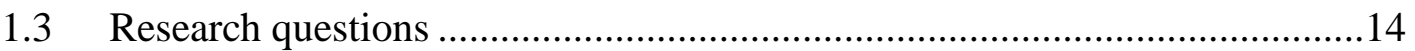

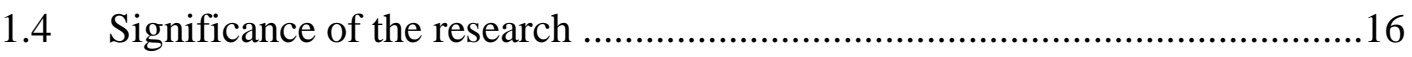

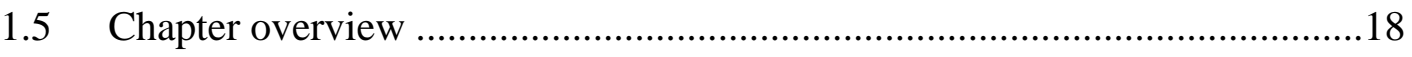

2 Literature review, methodologies, and methods ..................................................28

2.1 Agency, reproductive justice and digital technologies...................................29

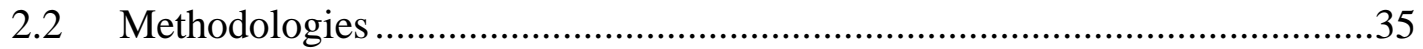

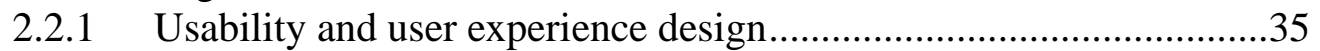

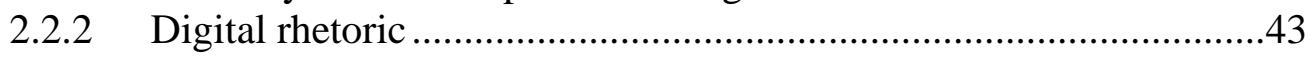

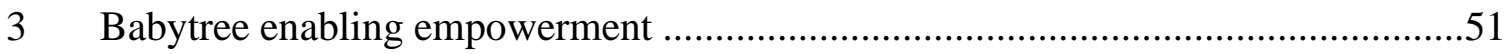

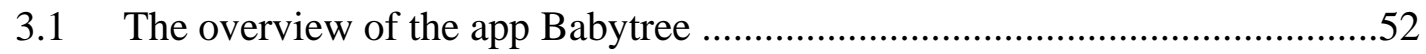

3.2 Digital style and Interfaces as an exordium to engage users...........................59

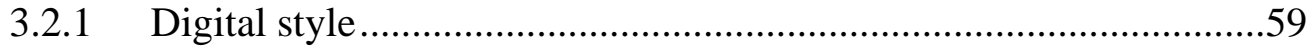

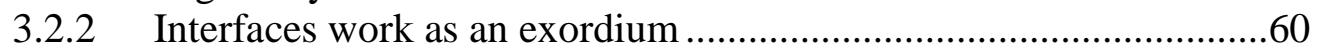

3.2.2.1 Using "Q\&A" to create multi-directionality....................62

3.2.2.2 Using the content-on-demand model to engage users' interests 66

3.2.2.3 Engaging users through various schemas of social

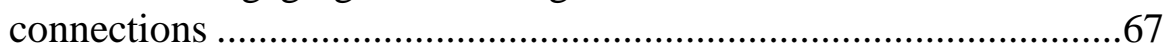

3.3 Using stylistic strategies to promote agency ...............................................70

3.3.1 Using second person narratives .......................................................

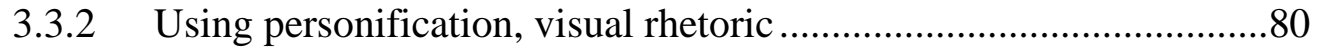

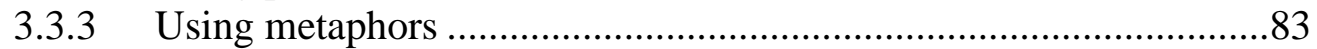

3.3.3.1 Risk management........................................................... 86

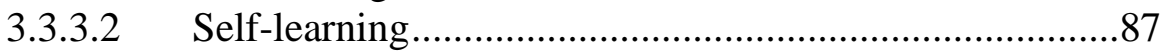

3.3.4 Using the first-person pronoun "I" .................................................. 
3.3.5 Using different colors .................................................................91

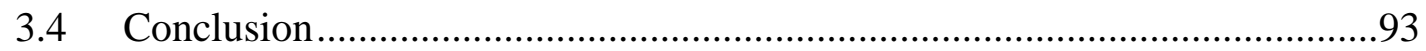

$4 \quad$ Using digital space to reclaim rhetorical agency ……..........................................99

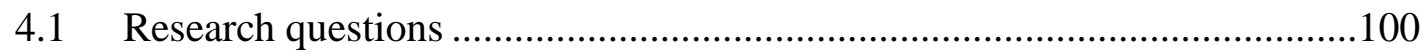

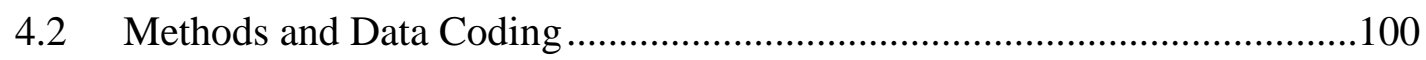

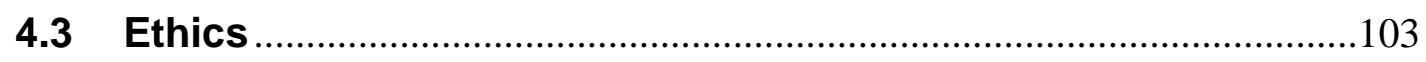

4.4 Reasserting Rhetorical Agency on the app Babytree ...................................104

4.4.1 Negotiating and resisting dominant medical practice and discourse 104

4.4.2 Fighting and compromising with the traditional culture..................111

4.4.3 Using personal experience as marketing rhetorical strategy ............116

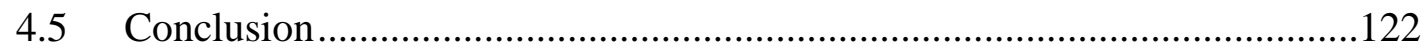

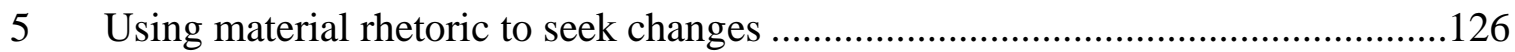

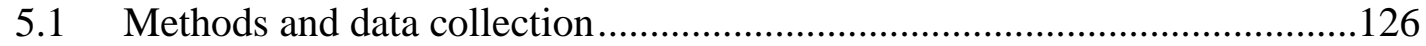

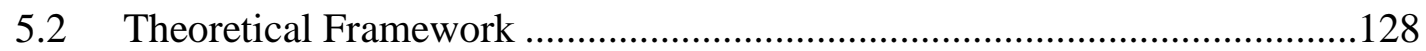

5.3 Using the craft of crocheting to identify with depressed mothers.................132

5.4 Sharing embodied experiences to assert their identity ..................................134

5.5 Using material rhetoric to seek meaningful changes....................................135

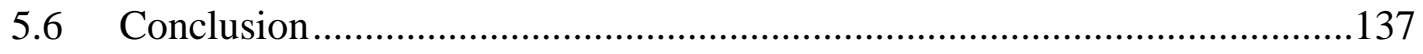

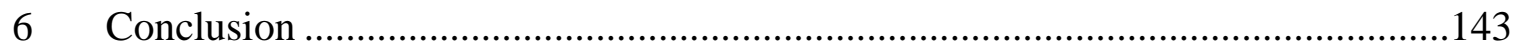

6.1 Research summary ...............................................................................143

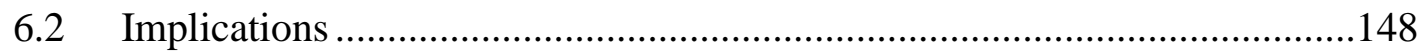

6.2.1 Theoretical implications................................................................148

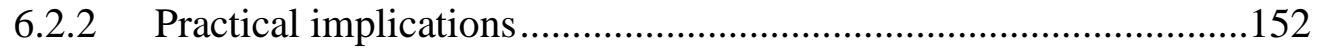

$6.3 \quad$ Future directions for scholarship ..............................................................156 


\section{Preface}

Chapter 4 is on how Chinese women users engage with the app Babytree by writing their embodied pregnant and mothering experience into online narratives and "selling" them to generate income, and the possibilities that they assert their rhetorical agency in the context of the app. Under the guidance of my advisor Dr. Marika Seigel, Dr. Erin Frost, and other anonymous reviewers, I wrote the chapter. Parts of this chapter will be published in Technical Communication Quarterly in July 2021.

Chapter 5 is on how a new Chinese mother with postpartum depression used the craft of crocheting to identify with other depressed mothers and they worked together to use their crocheted artifacts to do a large-scale installation to assert their rhetorical agency, raise the public's awareness, and combat the dominant biased culture that stigmatizes postpartum depression. Under the guidance of my advisor Dr. Marika Seigel, Dr. Lisa Melonçon, and Dr. Cathryn Molloy, I wrote this chapter. Parts of this chapter will be published as a chapter in the book Mental Health Rhetoric Research: Toward Strategic Interventions Ed. Lisa Melonçon and Cathryn Molloy in July 2021. 


\section{Acknowledgments}

This project would not have been possible without the guidance, support, and encouragement of so many people around me. First, I would like to thank my advisor Dr. Marika Seigel for her phenomenal mentorship. I am very lucky that she allowed me to pace my research and gave me space to develop my own questions but insisted that I argue about them in comprehensible ways. I am very grateful for her constructive and insightful feedback to improve my work and guide me through my confusion.

Thank you to my committee members Dr. Robert Johnson who inspired me to think about the relationship between rhetoric and technology, which has been a primary focus in my present and future research; and Dr. Sarah Bell who guided me to step into a totally new field for me: digital rhetoric that has been part of my research, too. I am also grateful to you guys for the time you invested in my research and job hunting. A special thanks goes to Dr. Kim Hensley Owens whose book Writing Childbirth inspired me to have this dissertation. I really appreciate you guys' insightful feedback as my work goes through revisions.

Thanks to Peter J. Ellenson for editing my drafts meticulously and painstakingly; to Dr. Lisa Melonçon, Dr. Cathryn Molloy, Dr. Erin Frost, and other two anonymous reviewers for their inspiring critiques that pushed me to rethink significant parts of my work.

I appreciate our department gracious support, especially when I was in hospital for an emergency surgery, Dr. Patricia Sotirin, Dr. Victoria L. Bergvall, Dr. Ramon Fonkoué, Dr. Shelly Galliah, Dr. Karla Kitalong, Ms. Jacqueline Ellenich, and other professors gave me cordial care and support in every possible way. I will be grateful for each one's support forever.

I am delighted to have met so many wonderful colleagues at Humanities Department and friends in Houghton who have been always supportive in my professional growth and personal life. Thank you everyone!

Finally, my heartfelt thanks go to my family, especially for my mother's understanding, my sister's and brother in-law's encouragement, and the joy that my nephews have brought to me. I appreciate my Chinese friends' assistance at Michigan Tech. Without them, I could not have accomplished this. 


\section{Abstract}

It is well known that China has implemented a Family Planning policy. Chinese women have limited options in reproduction, and their agency is constrained by the government, the medical institutions, and the traditional Chinese patriarchal and biased culture. By utilizing rhetorical analysis as a primary methodology with a focus on rhetorical agency, this dissertation analyzes two cases where digital technologies such as social media and apps facilitate users' rhetorical agency to counter instances of reproductive injustice. First, I focus on China's most popular pregnancy and mothering app, Babytree, to examine how the app rhetorically positions its users to enable empowerment and how users engage with the app by writing their embodied pregnant and mothering experience into online narratives and "selling" them to generate income, and the possibilities that they seek meaningful changes in the context of the app. Second, I investigate how a new Chinese mother with postpartum depression used the craft of crocheting to identify with other depressed mothers and they worked together to use their crocheted artifacts to do a large-scale installation to raise the public's awareness and combat the dominant biased culture that stigmatizes postpartum depression.

These Chinese women adopt different rhetorical strategies to integrate their embodied experiences into medical knowledge to attract users' attention and to recast their embodied experiences as material rhetoric to raise the public's awareness. They take advantage of technological affordances on the app and social media to combat dominant discourses and promote reproductive justice. I argue that these Chinese women economize and materialize their embodied experience of pregnancy and motherhood, which may alter gender oppression in patriarchal and biased cultural discourses and shows a powerful feminist rhetorical agency. Meanwhile, this research also aids in theorizing how reproductive injustice occurs in relation to institutional and cultural oppression in different cultures and groups, and how digital spaces are an ideal place for women to assert rhetorical agency in enacting intercultural communication of reproductive justice. 


\section{Introduction}

I begin with two stories: in August 2017, a pregnant Chinese woman's death sparked a hot debate in China's social media. The pregnant woman's family and the First Hospital of Yulin in Shaanxi province blamed each other for rejecting the woman's request to have a caesarian section, which allegedly led to her jumping to death from the fifth floor of the hospital. China Daily reported that the 26-year-old woman, Ma Rongrong, was admitted to the hospital to give birth on Aug $30^{\text {th }}$, a week before her due date. According to a hospital statement, medical checks showed that the baby's head was bigger than average, which suggested higher risks during natural birth, and therefore Ma's doctor advised Ma and her family that Ma should have a c-section. Her family refused and her husband signed a document at the hospital confirming that Ma would deliver naturally. Due to labor pain, she asked her husband to allow a c-section the next day. Her doctor and nurses also advised her husband to allow the surgery, but all requests were rejected. Possibly as a result of pain, Ma jumped off the top of the hospital building and died later that day. Her husband, Yan Zhuangzhuang, said in a statement that his wife had asked to have a c-section and that he had agreed with her about it, but the doctor checked her condition and said that she was going to give birth soon and did not need a c-section (Wang \& Li, 2017).

A few years later, in the early morning of April 27 in 2020, a 37-year-old woman died by suicide in Changsha in China; she leapt off a high building to her death while holding her 5-month-old daughter. It is reported that the deceased woman, Su, earned a 
$\mathrm{PhD}$ degree overseas, and the dead baby girl was her second child. After giving birth, Su developed symptoms of postpartum depression, but her family did not take her depression seriously. One of her family members, for instance, noted that they believed that her death was related to postpartum depression, yet, "didn't expect it to be so serious" (Cao, 2020). Due to her PhD degree, Su's death became a hashtag — \#37-year-old overseas female returnee with a $\mathrm{PhD}$ degree, with her 5-month-old daughter, jumped off the building to death\# — on Weibo, one of China's most popular social media sites. There were more than 1,600,000 clicks. The case fascinated the public. Many users did not understand why such a well-educated woman, with a good job and a happy family, could develop postpartum depression, and some users even said that higher education couldn’t save her from postpartum depression (\#37-year-old overseas female returnee with a PhD, with her 5-month-old daughter, jumped off the building to death\#, 2020).

Both the stories highlight extreme, and tragic cases of reproductive injustice, and cases where women's rhetorical agency was severely constrained: they could not make themselves heard. The debate about Ma's death on China's popular social media Weibo and WeChat mainly focused on patients' medical rights. Some users blamed Ma's husband and her family for not allowing a c-section, while others blamed the hospital, arguing that the doctors did not effectively communicate with the patient and her family. Officially — based on a regulation released by the State Council, China's Cabinetmedical institutions must get consent from patients and a signature from a family member before performing surgery, except for emergencies in which doctors can make decisions 
without consent. Many leading physicians, such as Dr. Gong Xiaoming, a gynecologist at Shanghai First Maternity and Infant Hospital, recognized that pregnant women should have the right to decide whether to have a natural birth or c-section. However, the reality in China is that in many cases, the decision to have a c-section is made by the patients' family members and the doctors (WeChat,2017). Ma's case represents the experience of a large fraction of Chinese women who cannot choose between natural or surgical delivery. Their rhetorical agency in this context is constrained or denied by the combined impact of China's laws and policies, medical institutions, and patriarchal cultural norms. The denial of agency as to the method of delivery is only one way in which Chinese women are denied reproductive freedom.

In the case of Su's death, her relative's and the public's confusion revealed a shallow understanding of postpartum depression — a non-psychotic depressive illness and a common complication after childbirth. Postpartum depression is characterized by feelings of extreme sadness, anxiety, trouble sleeping, mood swings, and so on. It can last for weeks, months or even more than a year and make it difficult for mothers to take care of their newborn baby and themselves (Mayo Clinic). In the article of "“"Doing the month" and postpartum depression among Chinese women: A Shanghai prospective cohort study", Ding et al (2019) state that 5-20\% of mothers in high-income western countries and $25-36 \%$ in lower-income countries suffer from this maternal mental health problem. In China, postpartum depression is increasingly prevalent and ranks No.2 after depression among China's top mental health problems. About $17 \%$ of new moms are 
diagnosed with severe postpartum depression, and about $85 \%$ of moms may experience depressive feelings after childbirth every year in China (Ni, 2018).

The Chinese users' reaction to Su's death is not all that surprising. Postpartum depression is a new concept in Chinese culture, and the public knows little about it. Many Chinese people do not see postpartum depression as an illness, as in the case of Su who had symptoms but whose family did not pay attention to her depression. Depressed moms receive little support from their families $(\mathrm{Ni}, 2018)$. In addition, most Chinese people stigmatize postpartum depression. They regard tearfulness, fatigue, anxiety and other symptoms of postpartum depression as a sign of a new mom's weakness or her negative feelings. Many women feel ashamed to acknowledge that they have a mental disorder, and they are not likely to seek psychological consultation and medical treatment $(\mathrm{Xu}$, 2019). Due to not getting consultation or medical treatment in a timely and appropriate manner, some depressed moms harm themselves and even die by suicide (Ni, 2018). The cases of depressed mothers' self-harm and suicide are increasingly appearing in the Chinese media, which incorrectly signals that postpartum depression is inevitably selfdestructive. The high-profile negative coverage of postpartum depression further stigmatizes postpartum depression and many depressed moms feel sad and scared to admit that they have postpartum depression.

In other words, both tragedies are examples of Chinese reproductive injustice, a term that I use to signal coercive laws, policies, and rhetorical practices restricting 
reproductive freedom, hegemonic medical reproductive discourse medicalizing reproduction, and gender inequality as a result of the dominant oppressive culture.

\subsection{What is reproductive justice}

The term "reproductive justice" combines the concepts of reproductive rights and social justice. It was coined in 1994, by SisterSong, a group of women of color, as an organizing framework to respond to American reproductive oppression (Ross, 2006). As a critical and theoretical framework, reproductive justice provides a lens through which to view the exploitation and manipulation of women of color through intersecting, strategic "systems of oppression based on race, class, gender, sexuality, ability, age and immigration status" (Forward Together, 2020, p.1.). In order to help women of color to gain reproductive freedom, the reproductive justice framework advocates women's right "have children, not have children, and parent the children we have in safe and sustainable communities" (www.sistersong.net). These three values emphasize legitimacy of women's reproductive rights and simultaneously prompt to identify social, economic, and health issues that result in an intersection of reproductive oppressions (Sanger,2004). Within the framework of these three values, advocates address issues of reproductive injustice that are intersecting, systemic, and structural, and impact women's reproductive freedom and decision-making. They promote both the individual and the collective agency of women of color. Following this framework, activists and scholars from various disciplines have articulated how reproductive regulation and exploitation of women's body are essentially related to gender, sexuality, racism, environmental toxicity, 
education, disability, and other issues that impact women's reproductive lives (Briggs, 2017; Gaard, 2010; Murphy, 2017). Similarly, in China, women's reproduction and their bodies are regulated and exploited, too. The following section investigates how Chinese women are subject to medicalizing childbirth care and the Chinese oppressive culture, constraining their rhetorical agency.

\subsection{Reproductive injustice and constrained agency in China}

It is well known that China has implemented coercive birth control to regulate its population by Family Planning Policies and the Law of People's Republic of China on Population and Birth Planning. Apart from the constantly changing family planning policies and the national law, China's medical institutions and traditional and biased Chinese culture also constrain women's reproductive freedom.

\subsubsection{Medicalization of childbirth care}

In the United States, modern medical science treats childbirth care as medical events. In her book The Rhetoric of Pregnancy, Marika Seigel (2014) examines prenatal care instructions to investigate how prenatal care is pathologized and the pregnant woman's body is treated as "a risky body." Similarly, in her book Writing Childbirth: Women's Rhetorical Agency in Labor and Online, Kim Hensley Owens (2015) traces the American reproductive history to examine how prenatal and childbirth care becomes medicalized and midwives are excluded from the dominant reproductive discourse. She 
argues that subject to the bio-medical discourse, American pregnant women's agency is extremely constrained. The same is true of the Chinese context.

With the central health authority modernizing medical science and technology, Chinese pregnant women's agency has been constrained increasingly. In 1949 when the People's Republic of China was founded, the Communist Party nationalized the centrally planned healthcare and emphasized basic public health. As for obstetric care, the partystate-owned public hospitals only provided "basic prenatal care and prevention of complications of pre-eclampsia, perinatal infections, and postpartum haemorrhage" (Hellerstain, Feldman \& Duan, 2015). This rudimentary reproductive healthcare treated pregnancy as a pathological and medicalized event, and pregnant women were treated as patients. A study conducted in 2004 and 2007 claimed that in Shanxi Province in China one of the reasons that women chose home births was due to their dissatisfaction with hospital care and fear of being in hospital (Gao, Barclay, Kildea, Hao, \& Belton, 2010). Although many women were not satisfied with the basic obstetric care, at least, they had agency in choice of home and in-hospital births at that time. In 1999, the home birth rate in five provinces of western China was 71.7\% (Liu, Yan, \& Wang, 2010).

In 1978, China began to switch from a state-controlled and planned economy system to a free-market economy system, which dismantled the centralized public healthcare system (Gao, Larsson, \& Luo, 2013). In the article "Internet use by Chinese women seeking pregnancy-related information", Gao, Larsson and Luo inform that from then on, the central government has launched several rounds of reform to replace a 
primary healthcare-orientated system that was funded by the central government with a market-oriented and intensive healthcare system. The central government cut healthcare funding and allowed the state-owned public hospitals to adopt a fee-for-service running model. They disclose that in order to modernize the medical and healthcare system, childbirth has changed from traditional midwifery service to bio-medical maternity service by introducing obstetricians, obstetric nurses and doulas who encourage costly expenditures. Consequently, the healthcare reform policies have made reproduction become a big profitable business in China. They argue that the public hospitals continue focusing on the medical aspects of reproduction: potential complications of childbirth, and normalized medical checkups and screening of pregnancy. For example, "women are expected to be seen in pregnancy every 4 weeks, until 28 weeks of gestation, every 2 weeks until 36 weeks, and weekly until birth” (p.731).

Meanwhile, public health policy began encouraging total hospitalization of births (Hellerstain et al, 2015). In order to promote in-hospital births, healthcare insurance was employed for coverage of hospital costs. For example, in rural areas, the New Cooperative Medical Scheme that was launched in 2004 increased health insurance from $11 \%$ in 2003 to $92 \%$ nationwide in 2007 (Gao et al, 2010). Due to health "insurance coverage for childbirth, patient costs, and doctor and hospital revenues" and in-hospital birth promotion, the hospital birth rate increased from 76\% in 2001 to $91.7 \%$ in 2007 (Hellerstein, Feldman, \& Duan, 2015; Gao et al., 2010). In 2011, few women gave birth at home(www.moh.gov.cn). Meanwhile the rates of caesarean delivery increased 
dramatically in China. In some urban areas, c-section rates even reached 100\% (Huang, Li, Chen, Wang, \& Zhang, 2004). Research shows that doctors played a key role in high rates of caesarean delivery and that women had little choices in vaginal delivery and caesarean delivery (Ji, Jiang, Yang, Qian, \& Tang, 2015). Some doctors regarded caesarean delivery as not only a more effective procedure but also a chance to increase income and avoid medical disputes (Hellerstein et al., 2015). Additionally, the dramatically increasing cost of caesarean delivery has greatly contributed to revenues for hospitals and healthcare providers (Feng, Xu, Guo, \& Ronsmans, 2012). The reformed healthcare system that promotes the extensive use of obstetricians, obstetric nurses, and doulas, and instrumental in-hospital births, primarily focus on medial aspects of reproduction, in which women are subject to bio-medical technologies with little agency.

\subsubsection{Chinese culture shaping reproductive behavior}

Apart from the bio-medical childbirth care, traditional Chinese culture profoundly shapes Chinese women's pregnancy and motherhood. In China, the Confucianism philosophy plays a central role in traditional cultural norms. It values a patriarchal system that determines a patrilineal family where male power was dominant. Having sons means the continuation of the family lineage and high social status of the family (Jin et al, 2007). Women had lower status in traditional Chinese society. In her article "Feminism in Transition: Chinese Culture, Ideology and the Development of the Women's Movement in China", Leung (2003) discussed how women were treated in the past. She states that "Women were seen as temporary members of their natal families and had no 
rights to property or inheritance. These rights were based on the primacy of patrilineal descent and patriarchal authority" (p.360). When women married, their families often gave some property and money as dowry to their husbands' families. Women who gave birth to daughters but no sons, were often blamed and even abandoned. In traditional Chinese society, women were often treated with cruelty and oppressed by the patriarchal culture. The son preference remains strong until today and continues shaping Chinese women's reproductive behavior. With the coercive implementation of Family Planning Policies since the 1970s, plus "widely available sex determination technology and low cost of sex-selective abortion" (Jin et al, 2007), the strong son preference triggered high rates of sex-selective abortion, causing severe sex ratio imbalance. In 1982, the sex ratio at birth was 108.5, but in 2000, it became 116.90 (Li, Wei, \& Jiang, 2006). The oppressive patriarchal culture lingering in Chinese society continues to constrain Chinese women's reproductive freedom and forces them to suffer the trauma of sex-selective abortion.

In addition, the traditional Chinese medicine theory, which is primarily based on the yin-yang of Chinese Taoist philosophy, also affects Chinese women's reproductive behavior. According to Taoism, "the human body, like the cosmos, can be fundamentally divided into a positive force (yang) and a negative force (yin) which are complementary to each other. Health is thus seen as a harmonious equilibrium between the yin and yang" (Lau, 2012. p.28). Historical texts in Chinese medicine used the dynamic dichotomy to explain women's reproductive diseases. "Any sign of imbalance would result in illness" 
(Cheung, 2009, p.232). In order to keep the body and the mind harmonious, Chinese pregnant women were admonished to follow strict dietary and behavioral restrictions. For example, pregnant women should avoid eating cold foods such as cherries, and ice cream because cold foods have yin qualities which can lead to miscarriage or bleeding. Imbalanced yin and yang could also affect women's emotions, as Lau (2012) explains that "Emotional problems are the result of an imbalance between yin and yang... too much joy impairs the heart, and fright causes systemic diseases" (p.28). In the month after delivery, women were most vulnerable, weakened by labor and blood loss. To heal, they were confined at home for 30 days, in a process known as zuo yue zi, or postpartum practice. During this time, they were required to follow dietary and behavioral restrictions. For example, women should remain in bed and eat certain foods to increase the production of breast milk. Activities like taking a bath, going outside, eating raw foods, and crying were forbidden.

In ancient times, pregnancy, labor and delivery, and the postpartum period did in fact put women in considerable peril, giving rise to precautions that gave them a sense of control (Wu, 2010). These outmoded practices, unfortunately, remain popular. Postpartum practice centers and services are widely available and advertised throughout China. Wang (2019) argues that "postpartum practice is being promoted by the Communist Party as a beneficial, natural, and uniquely Chinese practice during the Information Era", which means the government continues to "control and influence Chinese women's body and actions for its sustained legitimacy and prosperity" (p.11) 
and Chinese women's family members assisting postpartum practice are viewed as the continuation of advocating blood lineage, which "reflects the power hierarchy and reproductive politics of the family"(p.12). Put another way, through postpartum practices, Chinese women's reproductive behavior is regulated by the Chinese government and their family members.

Furthermore, the isolated and strict postpartum practices affect new mothers' psychological health. Research shows that postpartum depression is increasing dramatically in China and it is positively related to postpartum practices (Liu et al, 2014). However, the Chinese biased cultural discourse on postpartum depression silences sufferers and renders their pain invisible. This masking significantly impacts depressed women and their families. It leads to a collective unwillingness to accept postpartum depression as a mental disorder and stigmatization against it. Therefore, a rhetorical response to cultural invisibility becomes essential.

\subsubsection{Gender discrimination in employment}

Chinese women suffer employment discrimination during pregnancy and after childbirth. In Chinese society, women are seen to be more biologically and psychologically suited to raising children and are therefore prohibited from participation in social production as compared to men, during this critical period of life. Put another way, Chinese society is reluctant to acknowledge the truth that vast numbers of women lose their jobs following childbirth. A survey from Zhaopin.com in China showed that 
$40 \%$ of professional women lost their jobs following childbirth in 2012 ("2020

Workplace Mother Survival Survey Report,” 2020).

What's more, China's constitution and Party-state policies only entitle women to have the equal right to participate in social production, not guaranteeing their equal opportunity to participate in social production. Here "equal opportunity" means that when women want or need to participate in social production, they have access to positions and income that matches their working competence. However, the current social reality in China is that a woman who takes on family responsibilities, especially those who bear the responsibility of childbirth, cannot get such equal opportunities to participate in social production again.

From the above investigation, it is clear to see that the reproductive discourse in the Chinese context is unjust and oppressive. Compared with the American reproductive discourse, due to the distinct social, political, and cultural structures, the Chinese one displays unique differences, though in both contexts prenatal and childbirth care are treated as bio-medical events. In China, women's childbirth is not viewed as an individual human right. It is controlled, regulated, and shaped by the state laws and policies. Family policies restrict the number of children that they can have; the traditional Chinese culture such as son preference continues to push women into the patriarchal value system; and labor markets exclude, in some cases, the women in their reproductive period from social production. The reproductive oppression in China is institutional and cultural. In her article "Narrative Inquiry in Human-Centered Design: Examining Silence 
and Voice to Promote Social Justice in Design Scenarios," Natasha N. Jones (2016) claims that "both individual and collective praxis is necessary to address issues of social injustices that are systemic, structural, and impact groups of individuals rather than individual singularly" (p.474). Informed by her argument, in this research, I investigate how Chinese women take advantage of digital technological affordances to write their personal pregnancy and parenting experience into online narratives, and the possibilities to promote reproductive justice. With online circulation of their narratives, other women may get inspired and take the same action to address reproductive injustice collectively.

\subsection{Research questions}

With the emergence of Web 2.0 technologies, smartphones and social media, mobile health technologies that use social media and apps have been integrated into people's everyday lives and provided more convenient avenues for women users to retrieve health information and exchange their personal experiences of pregnancy, childbirth, and parenting (Thomas \& Lupton,2016; Lupton et al, 2016; Thomas et al, 2018). Feminist scholars have identified these digital spaces as peer support communities where women with constrained rhetorical agency on reproduction share knowledge and personal reproductive experiences, which may challenge or intervene in the dominant reproductive discourse and promote reproductive justice (Friedman and Calixte 2009; Haas 2009; Moravec 2012; Nesbitt 2009; Owens 2015; Vinson 2018). However, such scholarly practices on reproductive justice and mobile health technologies such as apps and social media are predominantly conducted in the western context. There is limited 
research on rhetoric and technical and professional communication in the arena of reproductive justice in the Chinese context.

The West and China present distinct cultural and institutional structures with respect to reproduction. A greater understanding of reproductive justice in the Chinese context will enrich the fields of the rhetoric of health and medicine and technical and professional communication and bring needed nuance and sophistication to the fields. Therefore, this dissertation focuses on two cases where digital technologies such as social media and apps facilitate rhetorical agency in their users to counter instances of reproductive injustice. First, I examine how China's most popular pregnancy and mothering app, Babytree, rhetorically positions its users to enable empowerment and amplify agency, and then I investigate how the users engage with the app and the extent to which they may make changes politically and economically and how they may empower peers in the context of the app. I argue that these women are savvy users, who make use of digital technologies, such as social media and apps like BabyTree, to do transformative practices and promote reproductive justice on a potentially global scale.

Second, I focus on a case study to scrutinize how a new Chinese mother Mao Wan with postpartum depression used the craft of crocheting to identify with other depressed moms on social media, and how they further identified with one another by sharing embodied experiences of postpartum depression. Through the embodied identification practice on social media, they had a better understanding of the mental disorder and reclaimed agency to speak back to stigma against postpartum depression in 
China. They worked together to do a public crocheting artifact installation to raise the public's awareness of postpartum depression. I argue that the crocheting artifacts along with written signs in the installation work as material rhetoric, rhetorically intervening in the dominant narratives of postpartum depression in China. When Mao Wan's story went viral on social media, it may empower users to promote reproductive justice.

By utilizing rhetorical analysis as a primary methodology, this dissertation will address the following two research questions:

- How and to what extent can mobile health apps like Babytree build communities and provide their users possibilities for rhetorical agency?

- How do Chinese women take advantage of digital technologies' (such as social media and apps) affordances to assert their rhetorical agency?

\subsection{Significance of the research}

Significance of this research mainly lies in three aspects. First, this research is situated in the Chinese context. Due to the influence of cultural and institutional structures, the rhetorical representation of medicine and healthcare regarding pregnancy and childbirth in the Chinese context is very different from that in the western context. In other words, the new insights and significant nuances that this research provides make the fields of rhetoric of medicine and healthcare and technical communication more complex and comprehensive. 
Second, this research complicates the conceptions of rhetorical agency in rhetoric and technical communication. Literature reviews on rhetorical agency show that nearly all the scholarship focuses on political assertion of rhetorical agency, arguing for asserting rhetorical agency as ways of negotiating, disrupting or challenging, or resisting the dominant discourse. In this research, I investigate, apart from these Chinese pregnant women's political assertion of rhetorical agency by writing their personal embodied experience into online narratives to respond to China's unjust reproductive discourse, some of the pregnant women enact their rhetorical agency economically as well by "selling" their personal embodied experience online to generate incomes. Traditionally, women are the reproducers of workers and citizens and have played a vital role in the economy and the state. Neoliberalism dismantles this traditional gender and sexual division and social and material support of women, which intensifies gender exploitation. In this severe competition, women must reorient themselves to extend their work. In this research, I examine how these Chinese pregnant women, who are usually excluded from labor markets or have limited choices in labor markets, use digital technologies such as social media and apps as tools to help position themselves to "sell" their embodied experiences in reproduction, which increases their human capital and helps them obtain self-resilience in labor markets. I investigate the extent to which these women practice transforms both politically and economically. Put another way, my study on rhetorical agency can be considered to do transformative practices at political and economic aspects, which will extend conceptions of rhetorical agency. 
Third, this research makes contributions to feminist rhetoric and intercultural communication studies. In the previous research, feminist scholars usually regard embodied experiences as knowledge, which challenges or rescripts the dominant discourse of knowledge, especially in pregnancy and childbirth, for example, in Writing Childbirth, Owens (2015) argues that women's birth stories and narratives are rhetorics of health and medicine, circulating online with standardized and institutionalized rhetorics of labor, which amplifies these women's voices (p.15). In this research, I examine Chinese women adopt different rhetorical strategies to integrate their embodied experiences into medical knowledge to attract users' attention and to recast their embodied experiences as material rhetoric to raise the public's awareness. I argue that these Chinese women economize and materialize their embodied experience of pregnancy and motherhood, which may alter gender oppression in patriarchal and biased cultural discourses and shows a powerful feminist rhetorical agency. This research also aids in theorizing how reproductive injustice occurs in relation to institutional and cultural oppression in different cultures and groups, and how digital spaces are an ideal place for women to exercise rhetorical agency in enacting intercultural communication of reproductive justice.

\subsection{Chapter overview}

This dissertation consists of six chapters. The next chapter, Chapter Two, provides a literature review, as well as an overview of the methodologies that I employ in 
this study. First, I discuss the concept of rhetorical agency and review the scholarship on it in the fields of rhetoric and technical communication. Following this, I introduce the methodologies that inform the research, especially the approaches of usability and user experience design and digital rhetoric, the scholarship that the combination of them is used, and how they work together to offer better interpretation of the app Babytree rhetorically positioning its users for engagement and empowerment.

Chapter Three examines how the app Babytree rhetorically facilitates its users for engagement. Firstly, I discuss a pregnant woman's user experience when using the app, then I focus on the four interfaces to analyze how they rhetorically function to engage with its users. Finally, I identify and examine the stylistic strategies such as metaphors, the first-person pronoun and the second-person pronoun used in the regular updates provided by the app. The positive construction of pregnancy and parenting may act out power relations, which may disturb or challenge the hegemonic power of the medical discourse and the traditional and biased culture in China.

In Chapter Four, I identify and analyze the rhetorical strategies that the users adopt in writing their embodied experiences of pregnancy and mothering into the online narratives and "sell" them to generate income. As the Chinese women share their embodied experience, they not only practice political and economic transformations but also emphasize a gender stereotype that provides a firm ground for approaching the anxieties of contemporary pregnant women and motherhood in China (article.zhaopin.com). Because pregnant women and new mothers are routinely 
discriminated against in employment, many lost their jobs due to pregnancy and mothering. In order to extend their work, these Chinese women reorient themselves by "selling" their mothering experiences to increase their income. Reproductive justice in this context is closely tied to economic opportunities. I argue that these women take advantage of mobile technologies, such as social media and apps like BabyTree, to seek changes and promote reproductive justice, which may empower other users.

Chapter Five is based on a case study. Framed by Burke's identification theory and material rhetoric, in this chapter, I analyze how a Chinese new mom Mao Wan with postpartum depression used the traditional craft of crocheting to identify with other depressed mothers on social media, how they further identified with one another by sharing their embodied experience on postpartum depression, and how they worked together to use their crocheted artifacts to do a large-scale installation as material rhetoric to enact their rhetorical agency, raise the public's awareness, de-stigmatize postpartum depression, and combat the biased cultural discourse on it. I argue that this case reinforces that these women are not passive technology consumers; instead, they make use of social media to present their voices, which may not only make changes but also promote reproductive justice. In this sense, they are savvy technology users.

Chapter Six is the conclusion discussing the theoretical and practical implications and the future directions of this research. 


\section{References}

Briggs, L. (2017) How All Politics Became Reproductive Politics: From Welfare Reform to Foreclosure to Trump. University of California Press.

Cao, W. (April 27, 2020) The 37-year-old female overseas returnee with a PhD degree, with her five-month daughter, committed suicide by jumping off the building. It's suspected that her death was due to postpartum depression. (H. Wang, Trans.). Retrieved from https://news.sina.com.cn/s/2020-04-27/dociirczymi8706381.shtml

Cheung, N. F. (2009). Chinese midwifery: The history and modernity. Midwifery, 25(3), 228-241. https://doi.org/10.1016/j.midw.2007.03.001.

Ding, G., et al (2019) "Doing the month" and postpartum depression among Chinese women: A Shanghai prospective cohort study. Women and Birth. 33(2) 151-158.

Feng, X., Xu. L., Guo. Y., Ronsmans, C. (2012) Factors Influencing Rising Caesarean Section Rates in China between 1988 and 2008. Bulletin of the World Health Organization. 90(1), 30-39.

Friedman, M., \& Calixte, S. L. (Eds.). (2009). Mothering and Blogging: The Radical Act of the Mommyblog. Demeter Press. 
Gaard, G. (2010). Reproductive Technology, or Reproductive Justice?: An Ecofeminist, Environmental Justice Perspective on the Rhetoric of Choice. Ethics and the Environment, 15(2), 103-129.

Gao, Y., Barclay, L., Kildea, S., Hao, M., \& Belton, S. (2010). Barriers to increasing hospital birth rates in rural Shanxi Province, China. Reproductive Health Matters, 18(36), 35-45.

Gao, L., Larsson, M., \& Luo, S. (2013). Internet use by Chinese women seeking pregnancy-related information. Midwifery, 29(7), 730-735. https://doi.org/10.1016/j.midw.2012.07.003.

Haas, A., (2009) "Wired Wombs: A Rhetorical Analysis of Online Infertility Support Communities." In Balir, Gajjala, and Tulley (Ed), Webbing Cyberfeminist Practice: Communities, Pedagogies, and Social Action (pp.61-84). New York: Hampton Press

Hellerstein, S., Feldman, S., Duan, T., (2015) China's 50\% Caesarean Delivery Rate: Is It too High? An International Journal of Obstetrics \& Gynaecology, 122(2), 160164.

Huang, P., Li, G., Chen, W., Wang, G., \& Zhang, Y. (2004) The Changing Rate and Indications of Caesarean Section Over 12 Years (H.Wang, Trans.). Academic Journal Guangzhou Medicine College, 32:57-99. 
Ji, H., Jiang, H., Yang, L., Qian, X., Tang, S. (2015) Factors Contributing to the Rapid Rise of Caesarean Section: A Prospective Study of Primiparous Chinese Women in Shanghai, BMJ Open, 5(11):1-9

Jin, X., Li, S., Feldman, M.W.(2007) Marriage Form and Son Preference in Rural China: An Investigation in Three Counties. Rural Sociology, 72(4), 511-536.

Jones, N.N., (2016) Narrative Inquiry in Human-Centered Design: Examining Silence and Voice to Promote Social Justice in Design Scenarios. Journal of Technical Writing and Communication. 46(4): 274-492.

Lau, Y. (2012). Traditional Chinese Pregnancy Restrictions, Health-Related Quality of Life and Perceived Stress among Pregnant Women in Macao, China. Asian Nursing Research, 6(1), 27-34. https://doi.org/10.1016/j.anr.2012.02.005.

Leung, A. S. M., (2003) Feminism in Transition: Chinese Culture, Ideology and the Development of the Women's Movement in China. Asia Pacific Journal of Management.20: 359-374.

Li, S., Wei, Y., \& Jiang, Q. (2006) Girl Child Survival in China: Past, Present and Prospect for Future (H. Wang, Trans.). Market and Population Analysis 1: 2-16.

Liu, X., Yan, H., \& Wang, D. (2010). The evaluation of" Safe Motherhood" program on maternal care utilization in rural western China: a difference in difference approach. BMC Public Health, 10(1), 566. 
Liu, Y. Q., Maloni, J. A., \& Petrini, M. A. (2014). Effect of postpartum practices of doing the month on Chinese women's physical and psychological health. Biological Research for Nursing, 16(1), 55-63.

Lupton, D., Pedersen, S., \& Thomas, G. M. (2016). Parenting and Digital Media: From the Early Web to Contemporary Digital Society. Sociology Compass, 10(8), 730743. https://doi.org/10.1111/soc4.12398

Moravec, M. (Ed.). (2011). Motherhood Online. Cambridge Scholars Publishing.

Murphy, M. K. (2017) What's in the World is in the Womb: Converging Environmental and Reproductive Justice through Synecdoche. Women's Studies in Communication, 40(2). 155-171.

Nesbitt, K. (2009). "Angel Babies: Women's Webs of Loss and Transformation" In Balir, Gajjala, and Tulley (Ed), Webbing Cyberfeminist Practice: Communities, Pedagogies, and Social Action (pp.43-60). New York: Hampton Press.

Ni, D. (July 12,2018) The Silent Struggle of Having Postpartum Depression in China. Retrieved from https://www.sixthtone.com/news/1002609/the-silent-struggle-ofhaving-postpartum-depression-in-china

Owens, K.H. (2015). Writing Childbirth: Women's Rhetorical Agency in Labor and Online. Carbondale: Southern Illinois University Press. 
Ross, L. J. (2009). Understanding Reproductive Justice. SisterSong Women of Color Reproductive Health Collective May 2006. Retrieved from https://d3n8a8pro7vhmx. cloudfront.net/rrfp/pages/33/attachments/original/1456425809/Understanding_RJ _Sistersong. pdf, 1456425809.

Sanger, A., (2004). Beyond Choice: Reproductive Freedom in the 21 st Century. New York City: Public Affairs. p. 19-47. ISBN 978-1586481162.

Seigel, M. (2014). The Rhetoric of Pregnancy. Chicago: The University of Chicago Press.

Thomas, G. M.; Lupton, D. (2016) Threats and Thrills: Pregnancy apps, Risk and Consumption. Health, Risk \& Society, 17(7-8), 495-509.

Thomas, G. M.; Lupton, D.; Pedersen, S. (2018) 'The Apply for a Happy Pappy’: Expectant Fatherhood and Pregnancy Apps. Journal of Gender Studies, 27(7), $759-770$.

Vinson, J. (2018). Embodying the Problem: The Persuasive Power of the Teenage Mother. New Brunswick, Camden, and Newark, New Jersey, and London: Rutgers University Press. 
Wang, K. (2019). “Reproduction with Chinese characteristics”: A Rhetorical-cultural Analysis of the Practice of Postpartum Confinement in Urban China (Master Thesis, University of British Columbia).

Wang, X. \&Li, W. (Sept. 7, 2017) Voices rise for painless childbirth, China Daily. Retrieved from https://www.chinadaily.com.cn/china/201709/07/content_31668016.htm

Wu, Y. L., (2010) Reproducing Women: Medicine, Metaphor, and Childbirth in Late Imperial China. Berkeley and Los Angeles: University of California Press.

Xu, W. Ed. Chen, S. (April 23, 2019) Mom born in the 1990s "crocheting for handrails" and calling on the society to pay attention to postpartum depression. (H. Wang, Trans.). Retrieved from http://www.bjnews.com.cn/news/2019/04/23/571191.html

2020 Workplace Mother Survival Survey Report. (May 15, 2020) (H, Wang, Trans.) Retrieved from https://henan.china.com/news/hot/2020/0515/253075849.html \#37-year-old overseas female returnee with a $\mathrm{PhD}$ degree, with her 5-month-old daughter, jumped off the building to death\# (April 28,2020) [Weibo]. (H. Wang, Trans.). Retrieved from https://s.weibo.com/weibo?q=\%2337\%E5\%B2\%81\%E6\%B5\%B7\%E5\%BD\%92 \%Е5\%A5\%B3\%Е5\%8D\%9A\%E5\%A3\%AB\%E5\%B8\%A6\%Е7\%9D\%805\%E4 
\%В8\%AA\%Е6\%9C\%88\%Е5\%A5\%B3\%Е5\%84\%BF\%Е8\%B7\%B3\%Е6\%А5\%

BC\%E8\%BA\%AB\%E4\%BA\%A1\%23\&Refer=SWeibo_box 


\section{Literature review, methodologies, and methods}

The rhetoric of health and medicine is a new sub-discipline within rhetorical studies. Scholars of health and medicine focus their study and research on practices in the health and medical field by exploring and adapting theories, methodologies, and research finding from various disciplines, such as the medical and health humanities, science and technology studies, cultural studies, communication studies, technical communication, anthropology, sociology, philosophy, history and so on. In order to explain the complex and high-stakes phenomena in public health, clinical health and bi-medical practices and research of this field, "rhetoric of health and medicine scholarship tends to employ mixed methodologies that integrate theories and methods from a number of scholarly traditions and research practices, ranging from cultural and critical theories such as materialisms to various forms of community engagement and participant observation to software-aided qualitative and quantitative analysis of large data sets" (Scott and Melonçon, 2017). This research builds on rhetorical scholarship related to the rhetoric of health and medicine with regard to reproduction, as well as scholarship on reproductive justice, usability and user experience, and digital rhetoric.

Light and Luckin (2008) argue that "(social) justice must be understood in the context of our lived experiences" (p.9). In a sense, to achieve social justice is to transform oppressed people's lived experiences. Jones (2016) states that agency is the key to transforming the human experience. In this chapter, firstly, I review how scholars are theorizing rhetorical agency in the fields of rhetoric and technical communication, then, I 
introduce the methodologies that inform the research and how I blend them together to interpret the app Babytree rhetorically positions its users to enable empowerment, how users take advantage of the digital technological affordances to assert their rhetorical agency politically and economically and promote reproductive justice.

\subsection{Agency, reproductive justice and digital technologies}

Agency has been a topic to rhetorical scholars and theorists who are trying to theorize it recently. According to Kenneth Burke's (1969) pentadic rhetorical framework, agency is a kind of human innate capacity to speak and act. Specifically, "rhetorical agency refers to the capacity to act, that is, to have competence to speak or write in a way that will be recognized or heeded by others in one's community" (Campbell, 2005). Early rhetorical theorists focused on speaking and writing, seeming to link rhetorical agency with the capacity to communicate, presumably with an intention of persuasion. In other words, traditional rhetoric relies on rhetors' agency to persuade audiences and it only focuses on the agency of an individual rhetor.

Cheryl Geisler (2004) claims that "agency does not lie in the hands of any one person at the proposed writing table, but rather lies in the interaction among them. It is a complex interplay" (p.112). She clearly states that agency emerges from interaction. Campbell (2005) states that agency is promiscuous and protean. She explains that "rhetors/authors, because they are linked to cultures and collectivities, must negotiate among institutional powers and are best described as 'points of articulation' rather than originators... They are "inventors" in the rhetorical sense, articulators who link past and 
present, and find means to express those strata that connect the psyche, society, and world, the forms of feeling that encapsulate moments in time. In this sense, agency is invention, including the invention, however temporary, of personae, subject-positions, and collectivities" (p.5). Both Geisler's and Campbell's interpretations of agency offer depth to the rhetorical process, suggesting performance and interactivity. In "What Can Automation Tell Us About Agency," Carolyn Miller (2007) highlights performance and interactivity of agency. She argues that "agency can be understood as the kinetic energy of rhetorical performance that is generated through a process of mutual attribution between rhetor and audience". She points out that "speaking strongly resists automation because we understand it intuitively as a performance, meaning that it is dynamic and temporal, that it requires living presence" $(2007$, p.145). She emphasizes that "Interaction is necessary for agency because it is what creates the kinetic energy of performance and puts it to rhetorical use. Agency, then, is not only the property of an event. It is the property of a relationship between rhetor and audience" (2007, p.150). In this sense, agency emerges as a result of social action, as Campbell (2005) claims that agency is "communal, social, cooperative, and participatory" (p. 5). Rhetorical agency is no longer a rhetor's possession or an agent's property, instead, it is identified as a kind of kinetic energy that emerges in rhetorical performance and interaction, a "negotiation of subject and effect", or "an emergent property of embodied individual" (Miller, 2007; Lundberg \& Gunn, 2005, p.88; Cooper, 2011, p.421). 
With postmodernism advocating "the decentering of the subject--- the death of the author/agent," agency is detached from the agent, which "signals a crisis of agency, or perhaps more accurately, for rhetoric, since traditional rhetoric requires the possibility for influence that agency entails" (Miller, 2007, p.143). Geisler (2004) states that the detachment of rhetorical agency from human agents is a kind of "fragmentation of agency," as Jessica Reyman (2018) claims that "with this fragmentation, agency is situated within the material conditions that shape rhetorical action rather than positioning agency as possessed by a single human agent or arising out of a fixed rhetorical situation" (p.117), just as Hellenbeck (2015) argues that agency is produced or achieved in dynamic and ever-shifting elements within or across material- rhetorical networks where agents are enmeshed. It "is contingent on a matrix of material and social conditions," seeking the possibilities for social action as a "diffuse and shifting ... social location and opportunity into and out of which rhetors...move" (Herndl and Licona, 2007).

The concept of rhetorical ecologies can better illuminate the relationship between ever-shifting materials conditions and enactment of agency within them (Edbauer, 2005). According to Edbauer, rhetorical ecologies mean that "rhetorical situations operate within a network of lived practical consciousness or structures of feeling" and rhetoric flows with dynamic interactions of forces, affects and associations (p.5). In other words, rhetoric is fluid and situational, and thus rhetorical ecologies are opposite Bitzer's (1968) rhetorical situation in which the elements of audience, rhetoric, exigence, constraints and text are already existent and discrete. Reyman (2018) argues that "A focus on rhetorical 
ecologies places rhetorical activity within dynamic networks, as a system of relations within interdependent and responsive environments, and has led to a growing awareness of agency as removed from the singular, static, and autonomous author, rhetor, or agent" (p. 116). Rhetorical ecologies understand communicative acts as dynamic, interactive, and ever-shifting elements within and across networks. Digital technologies, as part of the ever-changing material conditions, interact with human agents continually. As rhetorical practices on digital spaces have been part of human daily life, (rhetorical) agency is closely tied with digital technologies. Rhetorical agency emerges from dynamic interactions with increasingly changeable digital technologies, which affords more opportunities for agents to exercise agency.

Within the fields of rhetoric and technical and professional communication regarding pregnancy and mothering, scholars such as Koerber (2006), Seigel (2014), Owens (2015) and Vinson (2018) have done excellent work analyzing the relationship between rhetorical agency and rhetorics of reproduction. They probe the evolution of the dominant narratives regarding reproduction and pregnant women's and mothers' reaction to the hegemonic systems and acknowledge that agency, which refers to the capacity to act, is the key to transforming or overcoming reproductive oppression (Burke, 1969; Campbell, 2006). They argue for rhetorical agency as ways of negotiating, disrupting, challenging, and resisting the dominant narratives to achieve reproductive justice in America (Koerber, 2006; Seigel, 2014; Owens, 2015; Vinson, 2018). However, there still exist uncertainties in locating rhetorical agency within the ever-shifting material and 
social conditions, which can be seen especially in consideration of how mobile health technologies, especially social media and apps, have the potentials to empower users' agency to rhetorically engage with the reproductive injustice (Owens, 2015; Vinson, 2018).

Web 2.0 technologies that allow users to create, collaborate, interact and share in the process of creation and consumption, shifting users from consumers to participants, play an essential role in today's communication landscape. Due to enabling the collaborative creation and sharing of information, Web 2.0 technologies such as social media and apps enhance interactivity and possibilities to access more information. These participatory and user-generated content technologies increase users' rhetorical agency and transform the human experience in communication. Jones (2016) argues that "the pursuit of social justice is inextricably connected to transforming the human experience" (p.474). In this sense, digital technologies such as social media and apps are a potential tool to promote reproductive justice as the human experience or users' agency is mediated through participatory and user-centered design technologies.

Research on (social) justice and the human experience or users' agency mediated through digital technologies has been conducted in multiple disciplines. In the fields of rhetoric and technical and professional communication regarding pregnancy and mothering, some notable scholars have explored digital spaces as a potential site to promote reproductive justice. For example, in Writing Childbirth, Kim Hensley Owens (2015) shows how women who wish to give birth at home or have fewer medical 
interventions in childbirth are constrained by biomedical hegemony. She argues that website platforms provide a space for these women to critically reflect on their experiences of childbirth, "offering other women ways of understanding, writing their ways into, and asserting feminist rhetorical agency over their own birth experiences" ( $p$. 2). She claims that writing and sharing their birth experiences online is a rhetorical and feminist act that responds to the American dominant medical and professional discourse. Similarly, in her book Embodying the Problem, Jenna Vinson (2017) argues that social media is a savvy means of facilitating teen moms' rhetorical agency. She shows how young pregnant and mothering women are stigmatized and constructed as social problems by the dominant narratives of reproduction. Through the case of the \#NoTeenShame Campaign, she argues that the participatory nature of social media allowed some young moms to gain their resistance to the dominant narrative of teenage pregnancy publicly visible. She said that "for marginalized subjects who do not have direct access to those in power, social media seem to be key" (p. 132).

Owens, Vinson, and other scholars make clear that digital spaces are an effective place for marginalized women to combat reproductive injustice. These women's engagement with digital spaces provides them new perceptions of their bodies and parenting. This suggests that women's new understanding of their identities and bodies reshape the meanings and practices of pregnancy and parenting, as well as challenging or changing the traditional norms of motherhood and the provision of healthcare (Owens, 2015). With the current presence of mobile health technologies such as apps and social 
media surrounding pregnancy and motherhood, pregnant women and new mothers easily have access to health information and care. The following sections will focus on the Chinese context. I will examine how the app Babytree to rhetorically positions its users for engagement and how it defines pregnancy and parenthood alternatively, and how Chinese women engage with the app to assert rhetorical agency and advocate reproductive justice.

\subsection{Methodologies}

This research touches upon several fields of scholarship. Specifically, it draws on rhetorical analysis with a focus on rhetorical agency as the main methodology, and digital rhetoric, usability and user experience, technofeminisam, and feminist rhetoric provide secondary frameworks. In the following section, firstly, I explain how the approaches of usability and user experience, digital rhetoric, and technofeminism inform the research, then, I discuss the methods I used to collect data and ethics.

\subsubsection{Usability and user experience design}

The term usability originated from Jakob Nielsen (1993), who defines it with five metrics: learnability, efficiency, memorability, errors, and satisfaction. He states that "Only by defining the abstract concept of 'usability' in terms of these more precise and measurable components can we arrive at an engineering discipline where usability is not just argued about but is systematically approached, improved, and evaluated (possible measured)" (p. 26-27). Apparently, this engineering approach of usability focuses on the 
precise measurement of a product or system. Based on this definition, there are two categories of evaluation methods used for usability evaluation. One is inspection methods and the other is user evaluations. Inspection methods are usually conducted by experts who are guided by usability heuristics such as a set of principles or guidelines, while user evaluations refer to a lab-based testing, measuring the user task performance when they are using a product in a laboratory setting. Both of the categories of evaluation methods emphasize features of products or systems (Sun, 2012). In this sense, usability is just confined in the context of use, having nothing to do with sociocultural and other contexts. In other words, the engineering approach emphasizes usableness of a product or system, reducing usability to functionality testing. Under this framework of usability, users are excluded from technology design and their satisfactions are ignored (Sun, 2012). To date, this engineering approach of usability still has been influencing all forms of technology design.

In 1998, the International Organization for Standardization (ISO) published the definition of usability, which does not attribute usability only to a product or system quality, instead, it approaches the concept of usability more comprehensively-----emphasizing "the extent to which a system, product or service can be used by specified users to achieve specified goals with effectiveness, efficiency and satisfaction in a specified context of use" (www.iso.org/obp/ui). Although the definition was updated in 2018, the core components of it, such as effectivness, efficiency and satisfaction, were not changed. Put another way, the ISO definition of usability expands the scope of 
usability research by bringing contexts in use and satisfying users, but it still stresses functionality of a product or system.

Under the framework of usability focusing on functionality, it is believed that mobile health technologies can improve health interventions by delivering timely care to manage disease, enhance treatment, and mitigate health care costs by reducing physician visits, resources consumption and emergency room visits (Alwashmi et al, 2019; Hayes et al, 2014; Vashist et al, 2015). However, mobile healthcare involves interaction among multiple stakeholders like patients, medical workers, patient family members, etc. If mobile health technologies fail to achieve the effective, efficient, and satisfactory use in health interventions, stakeholders will give them up, especially patients who seek medical interventions. Research shows that many mobile health apps are not easy to use for diverse patients (Sarkar et al, 2016); according to the mHealth Economics 2017/2018 program in a global study, $83 \%$ of mobile health app publishers have less than 10,000 users (research2guidance.com).

\section{User/patient-centered design}

As usability studies are conducted in various disciplines including anthropology, sociology, and technical communication, more and more scholars and experts have realized that usability studies should expand the research scope. In 1986, the term "usercentered design" was introduced into usability studies with publication of "UserCentered System Design: New Perspectives on Human-Computer Interaction" (Norman, 
1986). User-center technology design means that users influence a design process by getting involved to make the design take shape. Unlike the usability engineering approach in which usability research is a small part of a large system, user-centered design focuses on the usability of design (Abras, Maloney-Krichmar \& Preece, 2004). Therefore, the role of users is elevated. In The Psychology Of Everyday Things, Norman (1988) offers four suggestions on how a user-centered design should be:

- Make it easy to determine what actions are possible at any moment.

- Make things visible, including the conceptual model of the system, the alternative actions, and the results of actions.

- Make it easy to evaluate the current state of the system.

- Follow natural mappings between intentions and the required actions; between actions and the resulting effect; and between the information that is visible and the interpretation of the system state (p. 188).

These basic suggestions in the engineering field display that users are at the center of a design and designers identify and address users' needs and meet their interests in a design process. Similarly, in the field of technical communication, Sullivan (1989) advocates that usability studies should include "the work of people who design systems, test them, develop educational materials, and study users" (p.256). In this new framework of usability studies, users are not a task performer for testing a product or system features; instead, they become part of usability studies. In this sense, technologies can better meet users' needs. For example, in mobile health technologies, the user/patient- 
centered design approach can address patients' specific needs in various contexts based on patient demographics, health literacy, types of diseases, different phases of treatment, etc. One of examples that have applied patient-centered design into practice is from the research conducted by Klashja et al (2010). They describe the design of an app that targets cancer patients, helping manage personal health information during treatment. The authors argue that this patient-centered design approach offered a more effective way to support users. Although the user/patient-centered approach can adjust a product or system design to users' requirements and expectations, it still focuses on accomplishing certain predetermined goals. Put differently, the user/patient-centered design approach emphasizes usefulness, fulfilling low-level tasks of a product or system.

Participatory design

Participatory design, which started in Scandinavia, switched "from a productoriented design to process-oriented design" (Sun, 2012, p. 35). This approach invites users to participate in the whole design process to affirm that the design is usable and meets their needs. Users are not only an active participant in the design but also involved in the decision-making (Johnson, 1998). Users and designers are in a collaborative relationship. It aims to achieve users' high-level performance by functional empowerment and democratic empowerment (Clement, 1996). Accordingly, usability studies shift "from evaluation of user actions to engagement with users' (Salvo, 2001, p. 273). In digital health technology, the participatory design approach is increasingly used in design to improve healthcare service. It can help identify patients' real or practical 
problems and design to address them, as Clemensen et al (2016) state that "participatory design is a multifaceted assessment tool that helps explore more accurately clinical requirements and patient perspectives..." (p.122). However, although users participate in the design and their knowledge is valued in the design process, this participatory design approach still strives for functionality of a product or system, emphasizing usefulness and usableness. Sun (2012) argues that "the engagement with users through a communicative and collaborative process should not be the final end. An activist vision would aim for emancipatory potential and social changes" (p. 37). With smartphones and other mobile digital technologies becoming part of people's everyday use, users are interested in not only usefulness of technologies but also how they integrate into their daily lives to enjoy the use. In the context of shifting the use to consumption and entertainment, usability research methods with emphasis on usable and useful aspects seem outmoded and the usability research scope must be expanded to meet users' emotional, aesthetic, pleasant, and entertaining needs (Green \& Jordan, 2002; Norman, 2004; Wright \& McCathy, 2005). Thus, digital (health) technologies with a focus on fulfilling tasks seem ill-suited.

User experience

The user experience design approach seems to bring much more human factors in design. According to Nielsen and Norman Group, "user experience encompasses all aspects of the end-user's interaction with the company, its services, and its products" (www.nngroup.com). Nielsen and Norman explain that 
The first requirement for an exemplary user experience is to meet the exact needs of the customer, without fuss or bother. Next comes simplicity and elegance that produce products that are a joy to own, a joy to use. True user experience goes far beyond giving customers what they say they want or providing checklist features. In order to achieve high-quality user experience in a company's offerings there must be a seamless merging of the services of multiple disciplines, including engineering, marketing, graphical and industrial design, and interface design (www.nngroup.com).

Peter Morville (2004) represents this with seven metrics: useful, usable, valuable, desirable, findable, accessible, and credible. He explains that this approach can make users have meaningful user experience and information must be:

- Useful: Your content should be original and fulfill a need

- Usable: Site must be easy to use

- Desirable: Image, identity, brand, and other design elements are used to evoke emotion and appreciation

- Findable: Content needs to be navigable and locatable onsite and offsite

- Accessible: Content needs to be accessible to people with disabilities

- Credible: Users must trust and believe what you tell them (www.usability.gov) 
This user experience approach informs my research on user experience with the app, but, in this research, I did not do usability testing to evaluate users' experience because I was not concerned with traditional measures of usability such as user satisfaction, efficiency, and learnability. Instead, in the next chapter, I outline the potential user experience of a pregnant user of the app with a focus on how the app engages users through different kinds of activities via various functions as well as its affordances that may facilitate possibilities for rhetorical agency. I combine the approaches of user experience with digital rhetoric to investigate the app's engagement with users and affordances. I combine these two approaches because digital rhetoric focuses on rhetorical functions of the app's interfaces, such as the layouts, the features of the functions, and the texts embedded in the interfaces. Such scholarly attempts have been conducted in computer and composition studies. For example, Teena Carnegie (2009) argues that an interface functions rhetorically to enable empowerment and enact control via three modes of interactivity. Collin Bjork (2018) promotes combination of usability testing with digital rhetoric theories in online writing instruction. He argues that usability theory tends to see interfaces as static objects manipulated by users, while digital rhetoric views interfaces as dynamic, real-time interactions (p. 4). Both Carnegie and Bjork emphasize the rhetorical functions of interfaces. Following their work, in this research, apart from examining how the app's interfaces engage users through interactions and rhetorically enable empowerment, I focus more on what symbolic representation and cultural implications the app conveys when a potential user engages with it. 


\subsubsection{Digital rhetoric}

Digital rhetoric, according to Eyman (2015), most simply refers to "the application of rhetorical theories (as analytic method or heuristic for production) to digital texts and performances" (p.44). In the field of digital rhetoric, Zappen (2005) lists the following primary research activities:

- The use of rhetorical strategies in production and analysis of digital text

- Identifying characteristics, affordances, and constraints of new media

- Formation of digital identities

- Potential for building social communities (p.319)

In Digital Rhetoric: Theory, Method, Practice, Eyman (2015) adds more to the list, which includes:

- Inquiry and development of rhetorics of technology

- The use of rhetorical methods for uncovering and interrogating ideologies and cultural formation in digital work

- An examination of the rhetorical function of networks

- Theorization of agency when interlocutors are as likely to be software agents (or "spimes") as they are human actors (p. 44)

As for users' identities and agency in digital rhetoric, contemporary digital rhetoricians mainly focus on how digital spaces constrain identity formation and solidify gender and racial stereotypes. For example, Kevin Brock (2010) argues that the digital 
identity is predetermined by technology developers. He states that "the user who constructs an electronic identity (or many) is not the arbiter of that identity's boundaries; instead, it is the developer(s) of the relevant technology that has assigned the limits to what a user can be or do with that technology" (n.p). Similarly, in "Body on $<$ body $>$ : Coding Subjectivity", Jennifer Bay (2010) claims that web spaces facilitate construction of certain identities by computer programming. She explains that "web spaces in which bodies are 'tagged' and take on mediative properties that construct subjects... Each site allows for certain kinds of cultural codes, which are invented and arranged by computer code and which function as the attributes or markup of the body" (p.154). Her argument indicates that users' subjectivity is regulated or controlled by computer code. Unlike Brock and Bay, in her article "The appended subject: Race and identity as digital assemblage", Jennifer Gonzale (2000) addresses racial issues and argues that digital technologies can reinforce or challenge racial stereotypes. She examines how digital racial roles are constructed online and how "visual representations extend or challenge current conceptions of racial and cultural identity and relation of power" (p.29). In her work, she emphasizes the limits of identities that digital technologies construct; meanwhile, she identifies the aspect that digital technologies, such as social media, can facilitate users to challenge racial stereotypes. In other words, her work addresses the relationship of racial stereotypes and technology use both negatively and positively.

The insightful scholarship that demonstrates constraints and empowerment of digital spaces can be augmented by the approach of technofeminism because this 
approach can better interpret the relationship between gender and technologies. In her book Technofeminism, Judy Wajcman (2004) examines the historical, social, cultural and economic contexts in which technologies are produced and used and women's negotiation with them. She denies considering the advancement of technology and gender relations within the dichotomy of good/bad or utopian/dystopian discourses, instead, she emphasizes the interconnection of technology development and feminist identity politics. The relationship between women and digital technologies is contingent and dynamic. The technofeminism approach offers a framework in which technologies are an avenue for women users to challenge socially and culturally constructed gender stereotypes and dominant discourses (Haraway, 1991). In other words, the technofeminism approach focuses on the positive potential of technologies that allow women users to do transformative practices. Taken together, the approaches of rhetorical analysis, user experience, digital rhetoric, technofeminism, and feminist rhetoric offer frameworks through which to interpret how the app rhetorically facilitates its users to enable empowerment and how women use the digital spaces to reassert their rhetorical agency, promoting reproductive justice.

In the following chapter, I give an overview of the Babytree app by rhetorically analyzing the user experience it provides to a pregnant user. Specifically, I first examine how the interfaces function as an exordium to engage users by increasing interactivity, and then analyze what stylistic strategies used in the app's daily and weekly updates and the icons with captions on the user's profile interface. 


\section{References}

Abras, C., Maloney-Krichmar, D., \& Preece, J. (2004). User-centered design. Bainbridge, W. Encyclopedia of Human-Computer Interaction. Thousand Oaks: Sage Publications, 37(4), 445-456.

Alwashmi, M. F., Hawboldt, J., Davis, E., \& Fetters, M. D. (2019). The iterative convergent design for mobile health usability testing: mixed methods approach. JMIR mHealth and uHealth, 7(4), e11656.

Bay, J. (2010). Body on <body>: Coding Subjectivity. In Bradley Dilger and Jeff Rice (Eds) From A to A: Keywords of Markup. U of Minnesota Press.

Bitzer, L. 1968. “The Rhetorical Situation.” Philosophy and Rhetoric 1(1): 1-14.

Bjork, C. (2018). Integrating usability testing with digital rhetoric in OWI. Computers and Composition, 49, 4-13.

Brock, K. (2010) Open source software and the construction of electronic identities. Presented at the Carolina Rhetoric Conference, Raleigh, NC.

Burke, K. 1969, A Grammar of Motives. California: University of California Press.

Campbell, K.K. 2005. "Agency: Promiscuous and Protean.” Communication and Critical/Cultural Sudies 2(1):1-19.

Carnegie, T. A. (2009). Interface as exordium: The rhetoric of interactivity. Computers and Composition, 26(3), 164-173.

Clement, A. (1996). Computing at work: empowering action by low-level users. 
Clemensen, J., Larsen, S. B., Kyng, M., \& Kirkevold, M. (2007). Participatory design in health sciences: Using cooperative experimental methods in developing health services and computer technology. Qualitative health research, 17(1), 122-130.

Cooper, M. M. (2011). Rhetorical agency as emergent and enacted. College Composition and Communication, 420-449.

Eyman, D. (2015). Digital rhetoric: Theory, method, practice (p. 177). University of Michigan Press.

Edbauer, J. 2005. "Unframing Models of Public Distribution: From Rhetorical Situation to Rhetorical Ecologies." Rhetoric Society Quarterly 35(4):5-24.

Geisler, C. 2004. "How Ought We to Understand the Concept of Rhetorical Agency? Report from the ARS.” Rhetoric Society Quarterly 34(3):9-17.

Green, W. S., \& Jordan, P. W. (Eds.). (2002). Pleasure with products: Beyond usability. CRC press.

Gonzale, J. (2000) The appended subject: Race and identity as digital assemblage. In beth E. Kolko, Lisa Nakamura, \& Gilberg B. Rodman (Eds), Race in Cyberspace (2750). New York: Routledge.

Haraway, D. (1991). A cyborg manifesto: Science, technology, and socialist-feminism in the late twentieth century. In Donna Haraway (Ed), Simians, cyborgs, and women: The reinvention of nature (p.149-182). New York, NY: Routledge.

Hayes, D. F., Markus, H. S., Leslie, R. D., \& Topol, E. J. (2014). Personalized medicine: risk prediction, targeted therapies and mobile health technology. BMC medicine, 12(1), 1-8. 
Herndl, C.G., and Licona, A. C. (2007). Shifting Agency: Agency, Kairos, and the Possibilities for Social Action. Communicative Practices in Workplaces and the Professions. Ed. Mark Zachry and Charlotte Thraills. Amityville, New York: Baywood, p.133-153.

Hallenbeck, S. (2015) Claiming the Bicycle: Women, Rhetoric, and Technology in Nineteenth-Century America. Carbondal: Southern Illinois University Press.

Johnson, R. R. (1998). User-centered technology: A rhetorical theory for computers and other mundane artifacts. SUNY press.

Jones, N.N., (2016) Narrative Inquiry in Human-Centered Design: Examining Silence and Voice to Promote Social Justice in Design Scenarios. Journal of Technical Writing and Communication. 46(4): 274-492.

Klasnja, P., Hartzler, A., Powell, C., Phan, G., \& Pratt, W. (2010). Health weaver mobile: designing a mobile tool for managing personal health information during cancer care. In AMIA Annual Symposium Proceedings (Vol. 2010, p. 392). American Medical Informatics Association.

Koerber, A. (2006). Rhetorical agency, resistance, and the disciplinary rhetorics of breastfeeding. Technical Communication Quarterly, 15, 87-101.

Light, A., \& Luckin, R. (2008). Designing for social justice: People, technology, learning. Bristol, UK: Futurelab.

Lundberg. C. and Gunn, J. 2005. "Ouija Board, Are There Any Communications? Agency, Ontotheology, and the Death of the Humanist Subject, or Continuing the ARS Conversation." Rhetoric Society Quarterly 35(4):83-105. 
Scott, J. B., \& Meloncon, L. (2017). Manifesting methodologies for the rhetoric of health \& medicine. In Methodologies for the rhetoric of health \& medicine (pp. 1-23). Routledge.

Miller, C. R. 2007. "What Can Automation Tell Us About Agency?” Rhetoric Society Quarterly 37(2): 137-137.

Morville, P. (2004). User experience design. Ann Arbor: Semantic Studios LLC, 6(2).

Nakamura, L. (2008). Digitizing race: Visual cultures of the Internet (Vol. 23). U of Minnesota Press.

Nielsen, J. (1994). Usability engineering. Morgan Kaufmann.

Norman, D. (1986). User centered system design. New perspectives on human-computer interaction.

(1988). The psychology of everyday things. Basic books.

Owens, K. H. (2015). Writing childbirth: Women's rhetorical agency in labor and online. SIU Press.

Reyman, J. (2018). "The Rhetorical Agency of Algorithms”. Theorizing Digital Rhetoric. Ed. Hess, A. and Davisson, A. New York and London: Routledge (Taylor \&Francis Group).

Salvo, M. J. (2001). Ethics of engagement: User-centered design and rhetorical methodology. Technical communication quarterly, 10(3), 273-290.

Sarkar, U., Gourley, G. I., Lyles, C. R., Tieu, L., Clarity, C., Newmark, L., ... \& Bates, D. W. (2016). Usability of commercially available mobile applications for diverse patients. Journal of general internal medicine, 31(12), 1417-1426. 
Seigel, M. (2014). The rhetoric of pregnancy. Chicago: The University of Chicago Press.

Sullivan, P. (1989). Beyond a narrow conception of usability testing. IEEE Transactions on Professional Communication, 32(4), 256-264.

Sun, H. (2012). Cross-cultural technology design: Creating culture-sensitive technology for local users. OUP USA.

Vashist, S. K., Luppa, P. B., Yeo, L. Y., Ozcan, A., \& Luong, J. H. (2015). Emerging technologies for next-generation point-of-care testing. Trends in biotechnology, 33(11), 692-705.

Vinson, J. (2018). Embodying the problem: The persuasive power of the teenage mother. New Brunswick, Camden, and Newark, New Jersey, and London: Rutgers University Press.

Wajcman, J. (2004). Technofeminism. Cambridge: Polity.

Wright, P., \& McCarthy, J. (2005). The value of the novel in designing for experience. In Future interaction design (pp. 9-30). Springer, London.

Zappen, J. P. (2005). Digital rhetoric: Toward an integrated theory. Technical Communication Quarterly, 14(3), 319-325. 


\section{Babytree enabling empowerment}

Research on digital health technologies, especially women's digital health technologies, mainly argues that digital health technologies such as apps and social media reinforce the traditional gender roles and ignore the fact that these digital health technologies can also change users' traditional understanding and perceptions of women's health. For example, Sanders (2017) argues that wearable and activity-tracking digital devices scrutinize and discipline the female body, reinforcing the normalization of femininity; Johnson (2014) considers apps and social media on pregnancy and mothering from the perspective of achieving health goals. She claims that these reproductive apps encourage users to "view apps and social media sites as a means to improve and monitor their pregnancies, health, and their children's development and health" (p. 330); Lupton and Thomas (2015) examine how the self-tracking pregnancy apps display the gendered nature and "endorse expectations around pregnancy behavior that reproduce heteronormative and gendered ideals around sexuality, parenthood, and consumption" (p.495).

However, these advanced digital technologies could bring new perceptions and behaviors to users, too, as Marissa Doshi (2018) states that women's health apps “could offer women new ways of seeing themselves by challenging traditional notions about healthy female subjects" (p. 184). In this chapter, I investigate how the app Babytree defines pregnancy and motherhood to empower its users, which may change their traditional perceptions of pregnancy and parenthood. Firstly, I present an overview of 
Babytree by introducing the company's objectives to build the platform; secondly, I briefly discuss a pregnant woman's user experience of engaging with the app; then I analyze how the stylistic features of the interfaces function rhetorically to persuade users and amplify their agency through three modes of interactivity; and finally, focusing on the app's regular updates and icons with captions on a pregnant user's homepage, I identify and analyze the stylistic strategies that the app uses to define pregnancy and parenthood, encouraging users' engagement and provoking agency.

\subsection{The overview of the app Babytree}

Babytree is the most popular app on pregnancy and mothering in China. The company profile says that it aims to provide diversified products and services to meet the core needs of Chinese young families for knowledge acquisition, social networking, recording the growth of babies, health and medical consultation, online prenatal education and early education, and consumption and shopping. Baby Tree has developed four sections around pregnancy and parenting in terms of content construction: pregnancy preparation, pregnancy, newborns, and parenting. Meanwhile, it provides suitable professional knowledge for users in the four different periods. In other words, in each period, the app provides different content accordingly. Taking the pregnancy stage as an example, the content is divided into more than 20 topics, including prenatal care instructions, diet, routine check-ups schedules, etc. When the baby is born, the topics of the content will change to confinement diet (diets during postpartum practices), newborn feeding, early childhood education, etc. At the platform, users can share valuable 
experiences, obtain positive and meaningful experiences about pregnancy, parenting, and baby growth, and solve their multi-level and multi-sided pregnancy and parenting issues at the same time. On the app, users can join various circles to build social connections and share their embodied experience. The participatory and community-based design nature of the app attracts users. Babytree has 9.2 million registered users and 22 million visitors each month, and more than 200,000 photos are uploaded to Babytree every day. The Baby Tree incubation platform provides a comprehensive one-stop solution for the pan-fertility cycle for young families in China (Wen, 2012).

The app Babytree is easy to use. I registered and created an account on March 6, 2019 for research purposes. Users can use their phone numbers to register and then get a generated user's name that users can edit. Nearly all the users use their generated names or edited pseudonyms that they choose. When doing registration, users can add more personal information such as gender, height, birthday, menstruation date, place of residence, the hospitals they visit, registration date and so on. All these items are not mandatory for users' registration. For example, I added my information of gender, height, and the date when I created an account when I did registration. A registered user can freely navigate the interfaces and read the information on the app. When a user logs in, she is directed to the homepage interface. On the homepage interface (see Figure 1), there are four menus at the bottom: "homepage", "chats", "shopping", and "I" (the user). These four buttons direct the user to the four interfaces. In other words, the app consists 
of four main interfaces with numerous sub-interfaces. Users can "jump” from one interface to another freely.

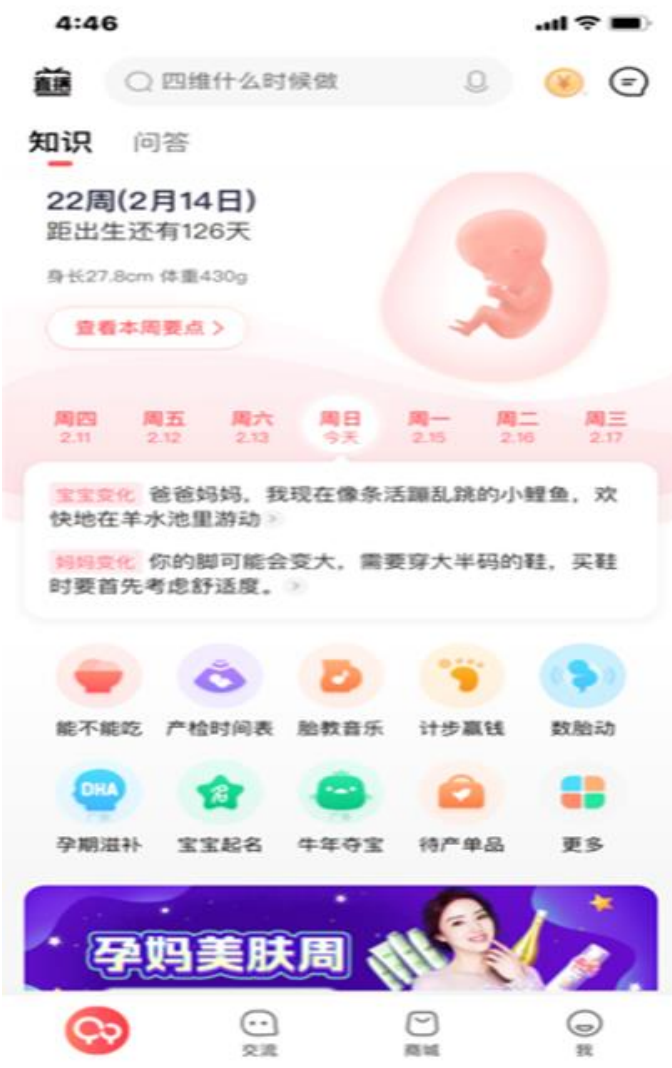

Figure 1. Screenshot of the homepage of the app Babytree

The app is useful and effective for pregnant women and new mom to obtain reproductive information and seek medical and peer support. The home interface provides information and knowledge about pregnancy and parenthood. On the top of it, there is a live stream button, a "search" button, and a "messages" button. The live stream button connects to a sub-interface where users can watch live stream talks given by 
healthcare providers. The talks consist of three categories, pregnancy preparation, pregnancy, and parenting. In the "search" entry, users can search information and knowledge about pregnancy and parenthood. Users can use the "messages" function to build connections with other users.

Below the above three functions, the homepage interface has two buttons named "Knowledge" and "Q\&A". Each one directs users to a sub-interface. In the "Knowledge" sub-interface, the app provides tailored knowledge about pregnancy or mothering, which includes daily and weekly updates about the details of the fetal development and the expectant mom's physical changes and expectations. This sub-interface also offers users functions ("my tools") by which they can check what food can be eaten, what can/can't be done, diet, how to read ultrasound images, and other advice and knowledge related to pregnancy. The "Q \& A" sub-interface is a place where users can exchange their pregnancy and parenting experiences with peers and consult with doctors for medical assistance by asking/ answering questions.

On the "Chats" interface (see Figure 2), there are more than 10 group themes such as pregnancy preparation, pregnancy, postpartum, same age, emotion, life, identity, illness, fashion, same city and so on. Click a theme icon, users can find sub-group topics around the theme. For example, if a user clicks the "pregnancy" icon, she will find subgroups like "routine check-ups", "what pregnant women can eat", "what pregnant women can't do," "gender prediction", "happy pregnancy", "stretch marks", etc. In this area, users can join the sub-groups and build a large social network. 


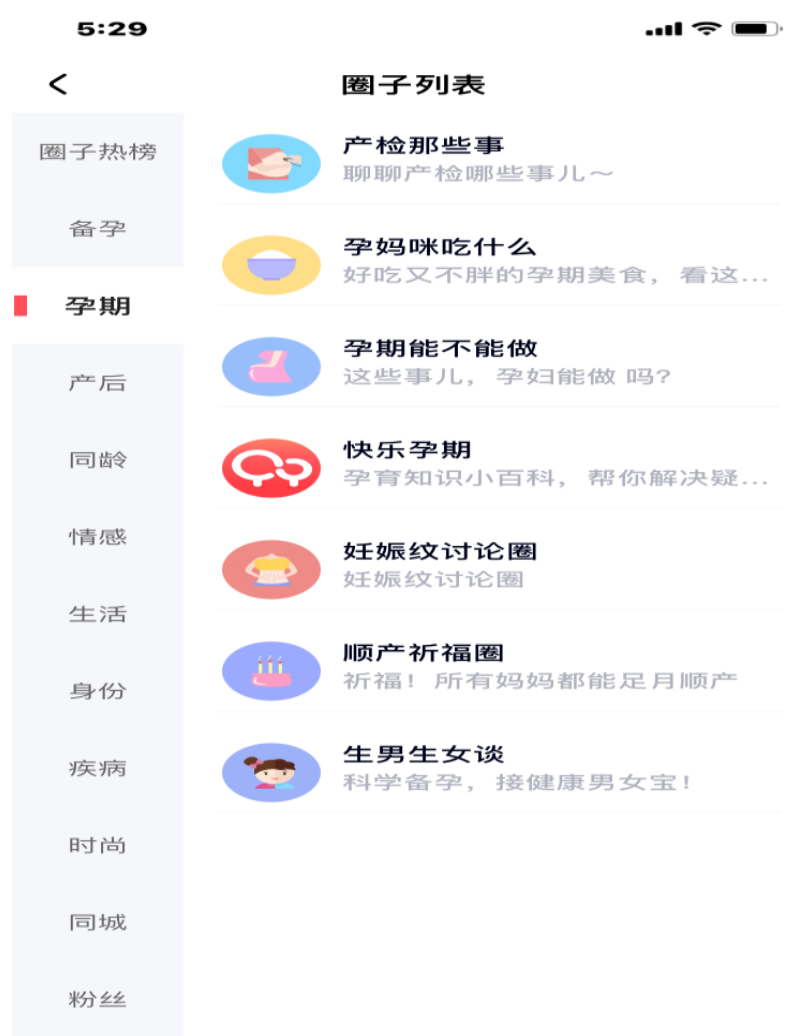

Figure 2: Screenshot of the "Chats" interface of the app Babytree

The "Shopping" interface provides various kinds of products related to pregnancy and parenting such as maternity supplies, toiletries during pregnancy, newborn supplies, pregnant women's clothing, nutritional products during pregnancy, baby toiletries, baby products, home daily necessities and so on. Users can do online shopping easily in this area.

The "I" button is directly connected to the user's profile page (see Figure 3) where users can edit their personal information, join the app's membership, and register for running a business store to earn incomes. There are six sections on this page: My 
baby, My tools, My perks, My community, My private circle, and Trends. The "My baby" section consists of two buttons: "pregnancy" that updates the pregnant time, and the "managing the baby" sub-interface records the detailed development of the newborn. The "My Tools" section has four buttons, and they are "pregnancy preparation", "pregnancy", "parenting" and "others". Take "pregnancy" as an example, this button connects with a sub-interface which offers the information, knowledge, suggestions, and medical consultation around pregnancy such as how to read ultrasound images, diet, fetal education music, the hospital bag, routine checkup schedules, counting movements, routine check-up consultation, medical professional consultation, etc. The "My Perks" section includes the buttons of "free trial", "online classes" around pregnancy and parenting, and "my orders". Each button directs the user to a sub-interface where she can do something accordingly. The "My Community" sub-interface is a place where users can communicate with other users and share their perks. In the "My Private Community" sub-interface, users can invite their family members and friends to socialize. In the "Trends" sub-interface, the user can find the updates of her following users and have interactions with them. This area is a place for the user to build social networking with other users. 


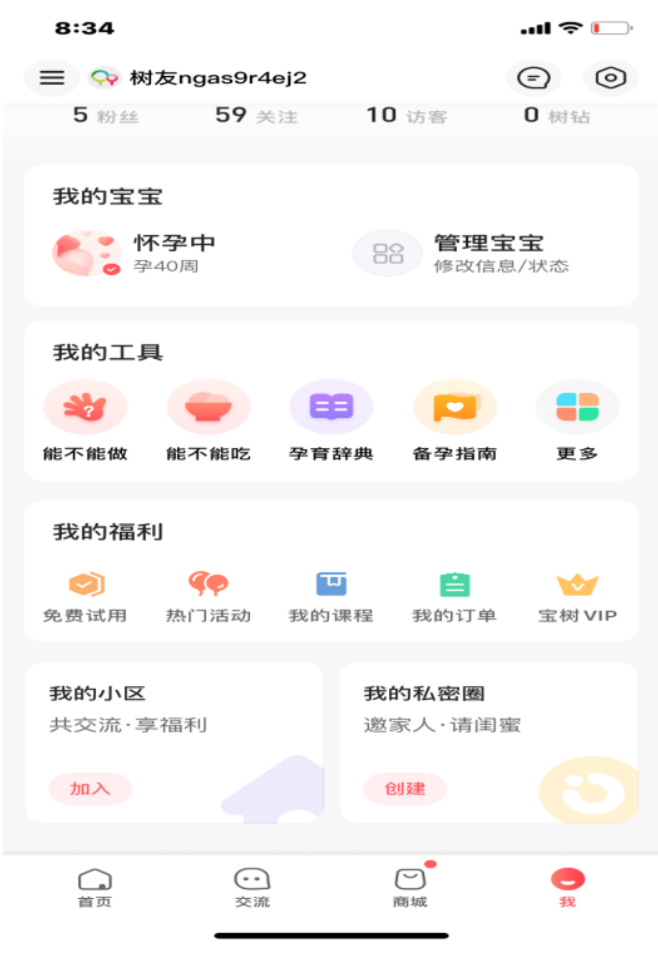

Figure 3: Screenshot of the "I" interface

The aforementioned displays that the app provides a large range of functions by which users can find information around pregnancy and parenting, build social networking, do online shopping, run online business, etc. Due to its portability on smart phones, it is convenient for users to have access to the app for information and knowledge anytime and anywhere. Users willingly engage with the app "to improve and monitor their pregnancies, health and their children's development and health" (Johnson, 2014, p.330). In this sense, the app changes users' behaviors and perceptions of pregnancy and motherhood, moving beyond traditional constructions of reproduction. In the following section, I focus on the interface design of the app to examine how the main 
four interfaces function rhetorically to enable empowerment by increasing users' highlevel interactivity.

\subsection{Digital style and Interfaces as an exordium to engage users}

\subsubsection{Digital style}

In classical rhetoric, style, as one of the five canons of rhetoric, addresses how to present ideas effectively. An excellent style needs four ingredients: correctness, clearness, appropriateness, and ornament, of which ornament is the most important. Aristotle states that "the whole business of rhetoric is concerned with appearance" (p.165). Apparently, style is an important element in rhetoric. According to Gideon Burton (2004), "style is not incidental, superficial, or supplementary: style names how ideas are embodied in language and customized to communicative contexts... to ornament meant to equip one's thoughts with verbal expression appropriate for accomplishing one's intentions" (n.p.). In digital rhetoric, style has been reimagined by scholars. For example, Eyman (2015) claims that "style is equivalent to "design"; thus, digital rhetoric must be concerned with understanding all the available elements of document design, including color, font choice, and layout, as well as multimedia design possibilities such as motion, interactivity, and appropriate use of media" (p.70). In this sense, style does not merely relate to persuasion but also ties with usability (Eyman, 2015, p. 70). Lanham (2006) narrows style down to "attention". He states that attention is essential to an information economy in the information era and digital style is about attracting attention. He advocates developing a "bi-stable way of examining an 
expressive surface, through for meaning, and at for style" (p. 256). Bradley Dilger (2010) disagrees that Lanham separates style from substance. He argues that "his (Lanham's) approach maintains the style and substance dichotomy. For me, it would be better to shift the definition of style ... to more fully acknowledge its connection to and inclusion of substance--- the commonalities of stylistic elements of all kinds, not only those manifested in surface features" (p.16). In this sense, in digital rhetoric, style restores the original purpose of classical rhetoric: effective persuasion.

Since style equals design, according to Eyman, and the elements of design are stylistic features, in what follows, I investigate the stylistic features in the interface design on the app. I adopt Teena Carnegie's (2009) approach of interface as exordium through three rhetorical modes of interactivity: multi-directionality, manipulability, and presence, to examine the stylistic features of the main four interfaces (the homepage interface, the communication interface, the shopping mall interface, and the user's profile interface), to see how the interfaces function rhetorically to engage its users.

\subsubsection{Interfaces work as an exordium}

Before introducing Carnegies' approach of interface as an exordium, I would like to briefly explain what an exordium is since it is excluded from the five canons of classic rhetoric and some readers may not know it. According to Cicero (1960), the exordium, along with the introduction, is the first component of arrangement. He explains that the exordium aims to make audiences "well-disposed, attentive, and receptive" (p.41). In modern and contemporary composition, the term exordium has been completely replaced 
by the term introduction which works as a hook to arouse audiences' interest in reading the text. In new media, Teena Carnegie (2009) suggests recovering the original meaning and function of exordium. She argues that "the exordium is not the introduction but the interface" (p.171). It works "to engage the audience not simply in action but in interaction" (p.171). She proposes three modes of interactivity used to determine an interface's degrees of interaction and engagement--- multi-directionality, manipulability, and presence (p.164).

The definition of multi-directionality originated from Sally McMillan (2006). It means new media facilitates "many-to-one and many-to-many" modes of communication. In the high level of interactivity in these modes of communication, the user is both a receiver and a sender and his or her messages are referred to by other users to create a responsive dialogue (Carnegie, 2009). Manipulability means that users can use digital objects such as sound, images, texts, videos, etc. to change the form and content on new media. If users can change the form and content to a higher degree, the level of interactivity will be higher. According to Carnegie (2009) and McMillian (2006), “a higher level of interactivity is created through a content-on-demand model. In this model, the user interacts to request information (as in search engines and databases) and to customize the interface" (p.168). The highest level of interactivity in the mode of manipulability is that users are allowed to create and add content (Carnegie, 2009).

Presence, according to Carnegie (2009), "is a product of integration of system attributes with user perceptions.... System attributes combine with users' perceptions 
such as immediacy, movement, and connection to create in users an experience of interacting socially and of being in a particular place or space" (p. 169). Presence consists of two aspects: social presence and spatial presence (Carnegie, 2009). Social presence means that new media provides "ways that the communication systems enable individuals to feel as if they are co-present even when they are not physically in the same place or time" (MaMillian, 2006, p.218). Spatial presence refers to "cyberspace" (Wardrip-Fruin \& Montfort, 2003, p.65). Users feel present in a place which is distinct from their real physical environment. New media provides schemas to map users' experience and actions and give certain responses. In the highest level of interactivity, users can navigate various schemas provided on new media, and at the same time they generate new schemes to engage with (Carnegie, 2009).

Carnegie (2009) concludes that through these three modes of interactivity, "it becomes possible to see how the interface as exordium defines users' action, determines the extent to which users can participate in dialogue and the creation of content and meaning, and positions users within certain social, political, economic, and cultural schemas" (p. 172). Based on Carnegie's approach, in what follows, I examine how the app's four main interfaces (the homepage interface, the circle interface, the shopping interface, and the "I" profile interface) function rhetorically to engage its users.

\subsubsection{Using "Q\&A" to create multi-directionality}


The interfaces on the app Babytree use the mode of multi-directionality to increase interactivity through the "chat" communication model. On the "Q\&A" subinterface of the homepage interface, users can ask peers and doctors questions related to pregnancy and parenthood. Peers' answers are quick and free of charge. Usually, a question can be answered by many users within a short time. For example, one user asked the following question:

"I gave a c-section birth one and half a year ago, can I have a second child now?" This question was answered by nineteen users within eighteen seconds. Through the many-to-one mode of communication, the user may obtain a reasonable answer based on these peers' embodied experiences. Importantly, all the users can read the question and all the answers to it, which shows a many-to-many mode of communication.

In addition, a user can ask a doctor for medical suggestions and assistance. She can ask a question in words with visuals or by phone with payment. How much she needs to pay for an online consultation depends on a healthcare provider's own pricing. The higher the professional level of a doctor is, the more expensive the charge is. But the charge is cheap compared with the payment for visiting a doctor in a hospital. For example, the average outpatient cost per time in public hospitals in 2019 is approximately $\mathrm{RMB}^{1} 290.8$ [\$45.4] (“Statistics on the development of health services in 2019”, 2020). The range of online charge on the app Babytree varies from RMB 39 [\$6] to RMB 99 [\$15]. More importantly, a user can consult with higher-level healthcare providers who

\footnotetext{
${ }^{1} \mathrm{RMB}$ is the abbreviation of Renminbi, the name of the Chinese currency.
} 
she cannot have access to in her local hospitals. Doctors can answer the question in a minute or within one hour. The asker is anonymous. All the doctors provide credible information such as their real name, the professional level, and the hospital where they work. The question is displayed on the interface. If other users want to listen to the doctor's answer, they will have to pay for it. For example, an anonymous user asked the following question,

I had a natural delivery in hospital and the baby was born on March 21st. I and the baby were discharged on the 23rd. Before the baby was discharged from the hospital, the doctor measured the jaundice index which was $189 \mu \mathrm{mol} / \mathrm{L}^{2}$ [the normal index for newborns is less than $204 \mu \mathrm{mol} / \mathrm{L}]$. The doctor asked me to come over on the 25 th to check the newborn. After the examination, the doctor said that the jaundice index was $370 \mu \mathrm{mol} / \mathrm{L}$, and my baby needed to be hospitalized with blue light treatment. Yesterday I went to the hospital and the doctor said that the jaundice index had dropped. It was $245 \mu \mathrm{mol} / \mathrm{L}$. Today the doctor said it dropped to $165 \mu \mathrm{mol} / \mathrm{L}$, but the doctor refused to let my baby leave the hospital and said that the baby needed to be observed for a while. I'd like to ask if there are any side effects of blue light treatment. Can my baby be discharged from the hospital today? If not, how long will it be hospitalized?

The asker paid RMB 59 [about \$9] for the doctor's medical assistance, and 40 users paid RMB 0.99 [15 cents] individually to listen to the answer. Through the "audit" function,

${ }^{2} \mu \mathrm{mol} / \mathrm{L}$ means micromoles per liter 
this one-to-one mode of communication expands into a one-to-many mode of communication, which not only helps build users' interactivity but also brings benefits to many users in medical care. It is worth mentioning that the interface offers special services such as "mental health counselling" through which users can contact psychiatrists by phone or messages. This service can be beneficial to users who are in rural areas or have difficulty accessing mental health counselling.

In the circle interface, a user can join various circles to build connections with other users across the country. In the circles, a user can exchange information with others. The interface enables users to "like", "comment" and "forward" others" posts. A user is both a sender and a receiver. She can also follow others' accounts to build personal relationships, knowing and responding to their posts timely. Through this responsive dialogue model of communication, "messages take on a more referential quality" (Carnegie, 2009, p.168). The interface also provides a "private message" function to users. By means of this communication mode, users can communicate with other users privately after they follow each other.

All in all, the interfaces with the multi-directional communication mode and the responsive dialogue model engage users in interaction with peers and medical professionals from different parts of China, increasing the level of interactivity. Meanwhile, users can efficiently have access to authoritative and credible healthcare professionals' medical assistance. 


\subsubsection{Using the content-on-demand model to engage users' interests}

Carnegie (2009) claims that the highest level of interactivity in the mode of manipulability is to allow users to create content. On the app Babytree, users' choices are constrained due to the packaged content model provided on the app. For example, on the user's personal homepage interface (the "I" homepage), a user can upload a video of her child to her profile. She can also invite her partner to take a profile picture. Although these predetermined functions fall under a packaged-content model and users have limited choices, they can appeal to users emotionally to build a virtual family atmosphere.

On the profile interface, a user can also create her own personal social circle where she can spread or share useful information. The interface provides users several modes to compose her post such as in an audio mode, or a video mode, or a written message mode, or a multimodal one with audio, words, and videos. All the posts that she creates also appear in the "trend" section on her profile interface. Although a user can create and add new content on her profile interface, her ability to manipulate or change the layout and design of the interface is limited and she is given limited power to construct herself as a user with a higher level of interactivity in the mode of manipulability. Because the app company encourages users to share useful information on pregnancy and parenthood by earning fees from traffic subsidies, some users take advantage of the opportunity to compose multimodal posts to attract more readers and generate more income, thus, in this sense, this engagement falls into a content-on-demand 
model and users can feel engaged and even empowered if they are interested in online writing. For example, in Chapter 4, I analyze how a user uses her embodied parenting stories to create posts with visuals and links as making rhetorical strategies to sell products, generating income.

\subsubsection{Engaging users through various schemas of social connections}

The app offers the "Q \& A" communication mode and social circles building functions to invoke social schemas of efficient healthcare support and assistance, increasing interpersonal communication. On the homepage interface, users can seek healthcare support related to pregnancy and parenthood. The peers" "Q \& A" interface is just like a chat room where a user can ask a question and others can answer it immediately. A user can also read all the questions and pick some questions that she is interested in answering. In this interface or digital space, it is convenient for users to seek peer support and suggestions. When a user asks a question, the interface reminds her how soon (usually within a few minutes) she will obtain answers, and after the question is posted, the interface will show how many people are answering this question within a certain time (usually a few seconds). Importantly, users can have access to all the questions and the answers and gain some useful information no matter where they are from. In other words, this digital space houses all the participants from various parts in China temporarily. The participants interact with one another and build social connections, which increases a high-level interactivity by getting involved with the social and spatial schemas facilitated by the interface. To some extent, this virtual 
communication is better than face-to-face interaction because it allows more users to answer questions and share their answers, which is beneficial to more users.

In the doctors" "Q\&A" interface, users can contact doctors from high-level hospitals by messages or phone calls. This communication model erases the spatial distance and establishes a momentary digital space where users can efficiently seek highquality medical care from healthcare professionals without geographical limits. Thus, when users have some medical issues that are not emergent or urgent, they can consult with an online healthcare provider instead of visiting a doctor, which is convenient and can help users save money. Through this mode of interaction, users increase their ability to access healthcare services and gain extra help from healthcare professionals before making their medical decisions. The distribution of healthcare enacted by professionals in the digital space can empower users so that they can reclaim their agency regarding gaining confidence and control over their healthcare decision making.

In addition, the circle interface facilitates building various social circles that users can join to build social networks. More than $70 \%$ of users on the app Babytree are aged between 25-35 and many of them are new (expectant) mothers who have anxieties about pregnancy, worries during pregnancy, and confusion about parenting, etc. (Wen, 2012). The circle interface offers various circles such as "the same age circle", "the same city circle," "the same hospital circle," and other circles with the themes like fashion, travelling, photography and so on. Users can join circles to share embodied experiences of pregnancy, childbirth, parenting, or talk about handicrafts, food, travel, beauty care, and other aspects of life. In these circles, users can connect with other participants from 
various areas of China, increasing their social connections. Users may feel connected to one another in the digital place when they share their knowledge and embodied experience.

In the digital place on the app, a user may experience high levels of interactivity when they engage with various circles, but her exploration remains contained within predetermined schemas. In other words, the user cannot map new schemas. Despite the constraints, the "Q \& A" interface engages with thousands of users every day.

Through analyzing the interfaces under the framework of three modes of interactivity, it becomes clear to see that the interfaces of the app as exordium position users, defining their actions and mapping their experience in several social schemas. When engaging with the app, users likely feel empowered by the high levels of interactivity that the interfaces facilitate. For example, by means of the affordances of the interfaces, users can share their embodied experiences of pregnancy, childbirth, and parenting to assist one another, and they can also have more choices to consult with higher professional levels of healthcare providers to obtain high-quality medical care. In this sense, the interfaces of the app facilitate users' engagement in various interactions with peers and healthcare providers, expanding their social connections and transforming their social and healthcare experience. Through the high-level interactivity the interfaces enable, the interfaces may not only amplify users' agency but also empower them in pregnancy and parenthood. In the following section, I turn to the language used on the 
app, especially in the daily and weekly updates and the function icons with captions, to examine what stylistic strategies are used to engage with users and promote their agency.

\subsection{Using stylistic strategies to promote agency}

Language matters. It shapes our attitude and cognition to understand ourselves, our body, and our status, especially our health. In this section, I investigate how the app reframes pregnancy and motherhood positively to empower its users through stylistic strategies used in the regular updates and the function icons with captions.

The daily and weekly updates and the icons with captions are important sources that the app uses to appeal to its users. They also reflect how the app alternatively defines pregnancy and motherhood. By analyzing the stylistic features in the daily and weekly updates and the icons with captions, this section reveals how the app provides advice, knowledge, and various kinds of support and encourages users to carry them out through their bodies, which may change traditional ways that pregnancy and parenting are perceived and practiced and produce new experiences of enacting pregnancy and parenthood. This section goes with the following questions to guide the analysis of the regular updates and the icons with captions:

- How do the regular updates (daily and weekly updates) define pregnancy? What impact may the regular updates have on the app's users?

- How do the icons with captions define pregnancy and motherhood? What impact may the icons with captions have on the app's users? 
- Do the regular updates and the icons with captions empower the app's users? If so, how?

Specifically, drawing on rhetorical analysis, this section analyzes what rhetorical devices or strategies and word choices are used in the daily and weekly updates and the function icons with captions to appeal to the app's users. Then, it examines what practices the regular updates and the function icons with captions promote to the app's users, and finally it investigates what outcomes users may achieve and whether they can enact their agency when following the regular updates and the practices suggested by the function icons. In order to better understand how the app appeals to its users, this chapter focuses on the researcher's (my) tailored daily and weekly updates and my own interface, where the icons with captions are displayed, to investigate how the app persuades a specific user rhetorically. The data used in the following section was from one hundred and fifty-four daily updates and twenty- two weekly updates (from March 6, 2019 when I registered in the app to February 14, 2020 when I collected data for the research) and all the icons with captions on the user's profile.

\subsubsection{Using second person narratives}

The function of "Knowledge" on the Babytree homepage displays the daily and weekly updates, which users can find immediately when they log in. The weekly update has six parts. They are the development of the fetus, the expectant mom's must-read, important matters, prenatal education, dietary suggestions, and what the expectant mom and dad do. The clear headings of the six parts make the updates very readable and 
accessible. The daily update has two parts: the changes of the pregnant woman and the development of the fetus. All the daily and weekly updates targeted to the expectant parents are second person narratives. The second pronoun "you" used in the updates may prompt users to check with themselves.

As a matter of fact, in order to engage with users, women's health apps adopt various kinds of rhetorical strategies to allure users. For example, Sophia Alice Johnson (2014) states that "Many apps for pregnancy (and mothering) are temporally tailored, meaning that the app is calibrating the pregnancy in terms of stages and time and encouraging the user to connect with her pregnancy as an exercise in fetal development" (p.335). The same is almost true of the app Babytree. The second person narrative used in the regular updates can provoke users to engage with the app.

In narratology, second person narrative means the emphasis of the narratee. Prince (1982) claims that the narratee is also the protagonist in the story s/he is told (p.84). Mary Frances Hopkins and Leon Perkins (1981) say that the "you" is "an actant". Similarly, in her article "Narrative "You" Revisited", Uri Margolin (1991) asserts that "the narrated you is a central agent in the sequence of events being recounted" and "the evens/action involving this "you" are specific and individual as regard their time and space, as opposed to the purely typical or recurrent" (p.6). Technical and professional scholars agree upon the narratological scholarship on the address "you". For example, Lesikar (1990) states that you-viewpoint writing emphasizes the reader's interests and concerns, and Campbell, Riley, and Parker (1990), based on speech act theory, argue that 
second person professional writing is directive, "to get the addressee to behave in some required way" (p.192). In her article "Barbies, Goddnesses, and Entrepreneurs:

Discourses of Gendered Digital Embodiment in Women's Health Apps”, Doshi (2018) states that the use of the pronoun "you" emphasizes individual agency. Informed by the above scholarship, I argue that the regular updates adopt the second person narratives to encourage users to actively engage in pregnancy and emphasize their agency in enacting pregnancy, health management, and a family's intimate bonding.

In the regular update on the app, the use of the second pronoun "you" or the second person narrative indicates that all the information is tailored to the user, provoking her to check with herself and know more about the changes of the fetus and her own body. Take the twentieth weekly narrative as an example: in "Developments of Your Baby", the user is informed of the development of the fetus and what the fetus looks like:

The growth of your baby is now stable. His hair is growing fast and he has eyebrows now. His small face looks close to that of a newborn. The structure of his cerebral cortex is forming, and the sulcus is increasing. His senses are developing rapidly. His senses of taste, smell, hearing and vision are in a critical developmental period. His retina is gradually forming and he is becoming sensitive to light now. If you shine a flashlight on your abdomen, your baby may respond to you in your tummy. 
The narrative uses "your baby" to emphasize the fetus like a real baby and "encourage a particular type of bonding and attachment with the fetus" (Johnson, 2014, p.338). The detailed description of the fetus development meets the user's curiosity of the development of the fetus. At the end of the narrative, it invites the user to interact with her "baby" by shining a flashlight on her abdomen, which may prompt her to actively engage with her body and the fetus.

Apart from the development of the fetus, "The Expectant Mom's Must-read" continues with the stress of "you", telling the user about her physical changes such as gaining weight and other medical issues related to pregnancy:

From now on, it is expected that your weight will increase by an average of $0.45 \mathrm{~kg}$ per week. If you are under-weight before pregnancy, you may need to gain more. Now, your uterus is getting bigger, squeezing your abdomen outwards, causing your belly to bulge outwards. Due to the enlargement of the uterus and compression of the pelvic veins, the blood flow of the lower limbs of pregnant women will not flow smoothly, which can cause edema of the legs, and edema of the back of the foot and the inner and outer ankles is more common. As the uterus squeezes the gastrointestinal tract and affects gastrointestinal emptying, you may often feel full and experience constipation. 
In addition, in the daily updates, the user receives similar information and suggestions to deal with uncomfortable physical changes. For example, the following second person narratives inform the user that edema is not related the amount of water she drinks, she should use a soft-bristled toothbrush if she has swollen gums, and how to relieve an upset stomach:

- Never deliberately reduce the amount of water you drink to prevent edema. Instead, keep adequate water.

- Your gums may become swollen. They may bleed when you brush your teeth. You can use a soft-bristled children's toothbrush.

- An upset stomach has been bothering you again recently. Drinking a cup of yogurt before meals or bedtime may relieve you.

When reading the stimuli of the above information, the user may unconsciously evaluate her body to see whether she has the same physical changes. If so, she may follow the suggestions to do specific practices. In other words, the user may willingly manage her health and build the bonding with the fetus according to the directions of the updates.

In "Important Matters", the weekly updates usually remind users of some medical issues that pregnancy may cause. In the twentieth weekly update, the reminders list three medical issues: 
Flat or sunken nipples: expectant moms who have flat or sunken nipples can now pull out the nipples by hand every day or use a nipple correction tool to correct them.

Pregnancy-induced hypertension syndrome: Pregnancy-induced hypertension syndrome is a syndrome in which a series of symptoms such as hypertension, edema, proteinuria, etc. appear after 5 months of pregnancy. It is necessary to insist on regular pregnancy checkups, pay attention to the signs of pregnancyinduced hypertension, and actively prevent pregnancy-induced hypertension.

Varicose veins during pregnancy: Varicose veins are a variety of purple forehead networks under the skin, usually on the calf or thigh. These swollen veins can rupture and bleed if they are injured, so treat them gently.

This section admits the risk of pregnancy, which aligns with the dominant medical discourse in modern medicine. As one of the reminders, when a user reads them, she may monitor and assess her body in order to achieve her health. In this sense, the app encourages pregnant women to be responsible for themselves by doing risk selfmanagement.

The weekly updates also give users some dietary suggestions. In "Dietary Recommendations" in the twentieth update, the narrative instructs the user how to eat healthy to make the fetus have sufficient nutrition: 
Recently, in order to increase your baby's nutritional intake, don't forget that "eating smaller meals" is healthy. You can divide the food you need to eat throughout the day into 5-6 meals, arrange additional meals between your lunch and dinner, and eat more mineral-rich foods, such as dark green leafy vegetables, meat, eggs, milk food, etc. to ensure the baby's nutritional supply.

This second person narrative is very directive, prompting the user to follow the specific dietary practice.

Importantly, this app includes expectant dads in enactment of pregnancy as well. Johnson claims that the information provided to expectant dads on pregnancy apps is usually gender stereotyped, appealing to "masculine-gendered activities such as providing information on how to prepare a nursery and deal with finances and insurance" (Johnson, 2014, p.344). In "Prenatal Education Time" and "What Should The Father-tobe Do" of the twentieth weekly update on the app Babytree, the expectant dad is encouraged to participate in prenatal education. The narratives firstly talk about the effective method of doing prenatal education, then state the significance of prenatal education, and finally teach the dad how to pick prenatal education materials for his wife and the fetus. The narratives say:

Dialogue is a very useful means of prenatal education. Although the fetus cannot understand what you say, the fetus can hear the voice and intonation of the outside world and feel the call from the mother. Moreover, it is very 
beneficial to use language to stimulate the development of the fetus's auditory nervous system and its brain. Therefore, the continuous stimulation of the fetus at this time will gradually improve the recognition ability of the fetus. The understanding ability of the fetus will continue to increase. With memory and education practices, the fetal will develop from unconscious existence to conscious existence.

For your baby to be smart and healthy, and for your wife to maintain a good mood, you, as a dad-to-be, should choose prenatal music and buy related books for your wife! You'd better choose more prenatal education CDs. Don't always listen to the same music and try to listen to various kinds of music as much as possible. In addition, as a dad-to-be, you can use your magnetic voice to gently sing some songs for your baby, which will promote the healthy growth of your baby.

From the statements that "For your baby to be smart and healthy, and for your wife to maintain a good mood, you, as a dad-to-be, should choose prenatal music and buy related books for your wife" and "as a dad-to-be, you can use your magnetic voice to gently sing some songs for your baby", it is clear that the app assumes the pregnant woman and the dad-to-be are a married couple, which appeals to the traditional heterosexual marriage practice and gets fathering practices involved in parenthood. Some scholars argue that fathering practices, such as "how to prepare a nursery, dealing with finances and insurance", and participating in prenatal education, suggest 
the changes in terms of sociocultural practices and expectation, but compared with mothering practices, "the mechanisms and devices to support these changes lag behind" and the mom still plays more important role in parenting (Johnson, 2014, p. 345; Lupton \&Barclay, 1997). Here I would like to say that Babytree's inclusion of fatherhood demonstrates that pregnancy is not merely a woman's individual event in China; rather, it is a couple's responsibility.

According to traditional Chinese family and gender values, the husband mainly undertook economic and productive activities while the wife was confined to the domestic sphere to give birth, raise children, take care of the elderly and other household chores (Hu \& Scot, 2014). This gender division of labor is still prevalent in China. The husband's role is to make money and he does not participate in taking care of the family, including caring for the pregnant wife and child-rearing. However, due to education, female employment, and exposure to western culture, young generations, who were born after the 1980s, are becoming less and less supportive of traditional family and gender values and striving for gender equality (Hu \& Scot, 2014). The husband is getting involved in domestic life such as housework and childcare (Li, 2018). Babytree targets the expectant father to get him involved in pregnancy, raising his awareness of fatherhood and exercising the family's bonding. In this sense, the app encourages gender equality in reproductive practices, though it normalizes heterosexual marriages. 
Because the updates take place every day and every week with the address of "you", "the pregnant woman is made to be constantly aware of her pregnancy" (Johnson, 2014, p.335). Put another way, the address "you" in the narratives creates self-referencing scenarios, ascribing agency to narratees/users and stimulating them to engage in pregnancy via the app. In the repeated process of self-referencing scenarios' stimuli, narratees/users are transported into those narratives and become protagonists/actants/agents getting involved in the promoted practices. These interactive practices with the app and the "baby" emphasize and amplify users' agency in enactment of pregnancy, health management, and a family's bonding.

\subsubsection{Using personification, visual rhetoric}

Many apps on pregnancy "encourage users to track the baby's progress", allowing users to "view the fetus' development during the period of pregnancy (Johnson, 2014, p. 339). For example, some apps enable users to upload 3D images of the fetus at different stages of pregnancy, and others allow users to imagine what the fetus is like in the wombs by playing users' distorted voice to the fetus or taking photos with distortion through the fetus' eyes (Johnson, 2014). The app Babytree goes a little bit further by allowing the fetus to "speak" to the expectant mom and uploading ultrasound and 3D images of the fetus to show what it is like in the womb at the specific moment. Through the rhetorical devices of personification and visual rhetoric, the fetus is personified and users can be provoked to "take care of" their "baby" willingly. 
Personification, as a figurative speech, gives human attributes to abstract or inanimate objects or in which abstract or inanimate objects are spoken of as if they were human beings. This figurative speech has been used widely, as Long (2009) states that "The attribution of human motivation, characteristics, or behavior to nonhuman organisms, forces of nature, supernatural phenomena, inanimate objects, and abstract concepts seems to be a natural human tendency, with a long tradition in poetry, myth, religion, and storytelling in particular" (p.25). In rhetoric, personification is an effective rhetorical device in speech and writing because it can enhance imagination, create engaging scenes, and build connections. In what follows, I investigate how personification and visuals are employed in the regular updates on Babytree to promote the fetus's personhood, encouraging users to build a virtual family bonding.

In the regular updates on Babytree, the fetus becomes a real baby, who "narrates" his/her growth to the expectant parents every day. For example, in the following daily updates, the baby said,

- Mom and Dad, I am now like a little carp, swimming happily in the amniotic pond (fluid).

- The uterus is getting more and more crowded, and I move around more frequently. Mom and Dad, I want a big "house".

- I can express my emotions. I will kick my mom's belly when I am happy and do the same when I am upset. 
- I often sleep at this time. My sleeping posture is very similar to that of sleeping after birth.

Here, the fetus is personified and can speak, telling the expectant mom what it is like and how it sleeps and expresses its emotions. The use of first-person pronoun "I" shows that the fetus has a digital identity, as Johnson claims that "babies (or fetuses) have an online birth prior to their physical birth" (p.338). The address "Dad and Mom" makes the expectant parents feel connected. To some extent, the fetus joins the expectant parents and they have a digital family now. Further, these narratives are striking and powerful, especially to the expectant mom, who can have an embodied and imaginative interaction with the fetus when reading the updates. Her excitement and happiness evoked in these updates can provoke her to engage in self-care in order to keep the fetus healthy.

In addition, the regular updates visualize the fetus by attaching ultrasound and 3D images to show what it looks like at various stages, which further encourages imagination of the fetus and strengthens connections and bonding with it. They also include visual images of fruits or vegetables to inform users of the approximate weight of the fetus every week. For example, in the twenty-third week of pregnancy, the fetus's weight is approximately equal to the weight of a potato. The attached 3D ultrasound image clearly shows the fetus's face, nose, eyes, arms and legs and the ultrasound image reveals the fetus' silhouette. These visuals encourage expectant parents to imagine their baby/fetus, eliciting "a particular type of bonding and attachment with the fetus" (Johnson, 2014, p.338). In other words, the fetus is not an abstraction any longer, rather, it is a real baby. 
Using ultrasound and 3D images "reinforces fetus individuality and personhood in a very visible and public way" (Johnson, 2014, p.338). Personifying the fetus as a real baby provides strong connections and emotions that can shift users' attitude toward pregnancy. When users associate the fetus' first-person narratives with ultrasound and 3D images, they will be provoked to have a new imagination and even stronger bonding, which further encourages expectant moms and dads to engage in health responsibility and management.

\subsubsection{Using metaphors}

Some apps on pregnancy and parenting provide users with various functions to monitor, assess, and manage their bodies, fetuses and babies. These functions include dietary and exercise suggestions, counting fetus movements/contractions, vaccine timers, feeding records, early childhood songs, and so on. Johnson (2014) and Doshi (2018) argue that with these functions, women users turn pregnancy and motherhood into a project management. Similarly, the app Babytree encourages its users to employ the functions to do project management in a metaphorical way. In the various function icons with captions on the user's profile, prenatal instructions and practices are metaphorically compared with technical and professional tools for a project, persuading users to use these tools to achieve the project completion successfully.

Metaphor, as one of the rhetorical devices, is used to compare a new idea to something that people have already known. In rhetoric, Quintilian compares metaphor with simile, and states that "the difference is that in simile something is overtly compared 
with the thing we wish to describe, while in metaphor one thing is substituted for the other" (ca. 95 AD, 8.6, 8-9). Aristotle highly praises metaphor. In Poetics, he says "by far the greatest thing is the use of metaphor. That alone cannot be learnt; it is the token of genius. For the right use of metaphor means an eye for resemblances" (1459a). In Rhetoric, he states that metaphor "sets the thing before the eyes" (1410). "And as Homer often, by making use of metaphor, speaks of inanimate things as if they were animate; and it is to creating actuality in all such cases that his popularity is due" (1411b). In 1980, George Lakoff and Mark Johnson published a groundbreaking book Metaphors We Live $B y$. In the book, they put forward the definition of conceptual metaphor and demonstrate that our abstract concepts are from our daily embodied experiences. In other words, we conceptualize an abstract domain by comparing it with something that we have already known and understood in our concrete world. Lakoff and Johnson posit that metaphor is not just a figurative term in language but related to human thoughts and actions. They explain that

"our ordinary conceptual system, in terms of which we both think and act, is fundamentally metaphorical in nature. The concepts that govern our thought are not just matters of the intellect. They also govern our everyday functioning, down to the most mundane details. Our concepts structure what we perceive, how we get around in the world, and how we relate to other people. Our conceptual system thus plays a central role in defining our everyday realities. If we are right in suggesting that our conceptual system is 
largely metaphorical, then the way we think, what we experience, and what we do every day is very much a matter of metaphor" (p.3)

Based on the above theoretical frameworks of metaphor, I analyze the icons with captions on a user's profile on Babytree to see how metaphor is used to structure pregnancy and motherhood and empower its users. On the profile page, as I mentioned in the overview of the app, there are 6 sections and one of them is the "My Tools" section. It consists of "Prenatal Preparation", "Pregnancy", "Parenting" and "Others". Here "My tools" are metaphors, indicating that pregnancy or parenting is a project and the prenatal instructions and practices provided in the user's profile are the tools used to complete the project. Comparing the prenatal instructions and practices with the tools to complete a project can materialize pregnancy and parenting, giving users a framework to understand them. With these tools, users can handle the project (pregnancy/parenting) well.

My analysis is focused on the two subsections of "Pregnancy" and "Parenting". "Pregnancy" has 20 icons with captions and "Parenting" has 24 icons with captions. Based on the descriptions (captions) of the icons, I identify the themes and group the 44 icons into 2 categories: risk management and self-learning. In the paragraphs that follow, I analyze how metaphorically structured concepts of pregnancy and parenting may impact users' thought and action, affecting their perception of pregnancy/parenting, fostering their agency as to how they should interact with the app in order to manage health and parenting. 


\subsubsection{Risk management}

In the Chinese dominant medical discourses and traditional Chinese cultural norms, women's bodies in pregnancy and during the postpartum period (one month) are taken as being risky, especially women who give birth to their babies must do traditional Chinese postpartum practices. The metaphor "tools" can create association with completing a project, then users will be led to seek certain tools to fix problems/risks during the process of finishing the project.

Based on the icons' captions, the tools that the app offers can fix several types of risks. Firstly, they can fix risks caused by diet and exercise. Both the "Pregnancy" and the "Parenting" sections provide pregnant women and new mothers with food and exercise suggestions around pregnancy and the postpartum practices. In the "Pregnancy" section, there is an icon that states "what can be eaten or not", one icon named "what can be done or not", and one icon promoting "pregnancy meals". If a pregnant user is not sure whether she can eat some specific food or do some specific exercises, she can check out with the tools. Similarly, the "Parenting" section has three icons around diet and exercises, too. They are "what can be eaten or not", "what can be done or not", and "postpartum meals", helping women who are doing postpartum practices to avoid dietary and exercise risks.

Secondly, the tools also help handle medical risks of pregnancy. The "Pregnancy" section provides several icons (functions). For example, with the "routine check-up schedules" function, users can assess the fetus' development and 
pregnant women's physical changes; the "counting fetal movements" function provides users a method to record the fetus' kicks and make sure the movements are normal; through the "Q \& A" function, users can ask doctors or peers for suggestions. With these tools, users can monitor and assess themselves to alleviate and even fix risks throughout the process of pregnancy. Put another way, in order to achieve the best outcomes in reproductive, fetal and infant health, pregnant women do not reject medicalization of pregnancy, instead, they embrace it and actively engage with the app to seek solutions to medical issues (Johnson, 2014; Lupton, 2011; Root \& Browner, 2001; Longhurst, 1999). Meanwhile, the app encourages traditional Chinese cultural practices such as postpartum practices.

\subsubsection{Self-learning}

Many users on the app Babytree are first-time mothers-to-be or first-time new mothers. They lack medical knowledge and expertise on pregnancy and parenting. The metaphorical framework of "My Tools" is able to help envision pregnancy and parenting as self-learning practices, thus users can turn to the tools to improve biomedical literacy in order to achieve best outcomes in the project (pregnancy/parenting) management. In "Pregnancy" section (see Figure 4), the icons with captions include "how to read ultrasound images" by which users can find information of ultrasound images at various stages; "reproduction dictionary" in which users can look up information about prenatal preparation, pregnancy, childbirth, and parenting; and "knowledge bank" where users can read all 
information about prenatal preparation, pregnancy, childbirth, and parenting. In "Imperial concubine promotion", users can test what they have learned from "reproduction dictionary" and "knowledge base". "A dictionary" and "knowledge bank" are items that people have had embodied experience with when doing schooling, thus users can have a direct understanding of the self-learning process about pregnancy via these tools. Users can also learn what to pack in a hospital bag for delivery from "hospital bag checklist". In addition, the tools provide information beyond prenatal instructions and practice but are very important for reproduction. For example, from the icon "hospital registration", users can find out what local hospital they should go to register for routine checks and giving birth. 


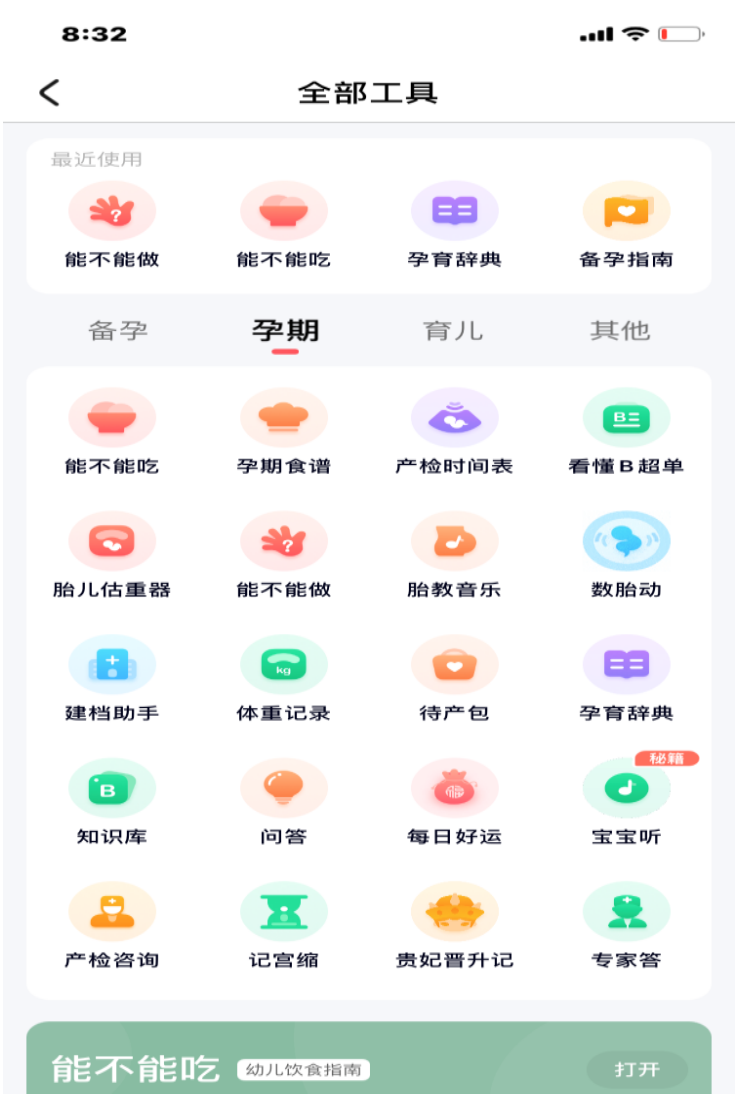

Figure 4: screenshot of "My Tools"

Another important tool in understanding parenting as self-education is tracking activities (like taking notes). In "Parenting" section, there are several icons (functions) with the texts like "baby's weight recording”, "feeding recording", "growth archive", "growth assessment", "baby's height and weight", and "vaccine schedules". These texts (functions) encourage users to "take notes" regularly in order to learn more about their babies. Frazier (2012) argues that tracking children's development can make "parenthood a more quantifiable, science-based endeavor" (p.28). I argue that tracking or recording activities not only make it easy for users to 
better understand their baby's development but also lend a sense of agency to them to professionally and technically practice parenthood, though Johnson (2014) argues that tracking activities produce "a new form of calculability. This instrumentalizes the body in a particular way..." (p.342).

\subsubsection{Using the first-person pronoun "I"}

As I have discussed in Chapter One, Chinese women's agency is constrained by the dominant medical discourse and the traditional Chinese postpartum practices. Pregnant women's bodies are pathologically risky and they are treated like objects. After childbirth, new moms are confined at home and follow strict postpartum practices. On the app Babytree, the use of the first-person pronoun to explain the functions in the profile page gives users a sense of agency, prompting them to freely navigate in all the functions.

Baker (2011) claims that first-person point of view is essential to ascribing human agency. She argues that "a first-person point of view and an agent are intuitively connected" and "the agent reasons about what to do on the basis of her first-person point of view" (p.4). In the user's profile page on Babytree, the first-person pronoun is used in all the functions' headers to appeal to users. They are "My Baby", "My Tools", "My Perks", "My Community", and "My Private Circle". In "My Baby", there are two subheaders (functions): "During pregnancy" and "Managing the baby", in which users can check information about pregnancy and mothering. In the "My Tools" section, there are icons (functions) with captions "prenatal preparation", "pregnancy", and "parenting". By these functions, users can find information, knowledge, suggestions about reproduction, 
postpartum practices, and parenting. In the "My Perks" section, users can obtain free online classes on pregnancy and parenting, do online shopping and check their orders. "My Community" provides an opportunity to users to join the local group for mothers-tobe and new mothers who can exchange their embodied experiences on pregnancy and mothering and share online shopping perks. Users can also build "My Private Circle" to invite their family members and close friends to socialize. When users enter their own profile page, the first-person pronoun "I" used there gives them a feeling of control or ownership, increasing their subjective awareness of all the functions, which may prompt them to engage with the app actively and freely.

\subsubsection{Using different colors}

Unlike most pregnancy and mothering apps that merely focus on maternal health and the fetus/baby development, Babytree also encourages consumption and entrepreneurship, which can be seen on the two icons (functions) in bright color under the user's name on the profile page. In other words, the two icons are kept on the top of the page to gain users' attention. When the user checks her profile page, she can immediately identify these two colorful icons (functions): a yellow one and a pink one. The yellow one is with the caption of "Joining the membership". When the user clicks it, she will be directed into another interface where she can pay to take online classes on pregnancy and parenting from medical professionals. If the user joins Babytree's membership, she will win some perks such as free and discounts of some online classes. Simply put, the app 
encourages users as consumers to develop/buy specific scientific and medical knowledge on pregnancy and parenting.

The pink icon (function) pushes users, especially stay-at-home new mothers, to run an online store by the text "being a mom store owner". After clicking the pink icon, the user enters an interface where there are several kinds of promotions with eye-catching headers to allure users to run an online store. For example, "purchase any spree, you will become a mom store owner", "Becoming a mom store owner, you will enjoy super privileges" such as "save money when making purchases here" and "make money when sharing your purchases". At the bottom of this page, there is a section with a header of "real store owner's feedback", in which three moms share their successful business experience. All of them are stay-at-home mothers. Here is an example,

After getting married, I look after my kid at home. Now I am taking care of my kid and at the same time I'm an owner of a retail store on Babytree. I can make money without being restrained, and I can also get bonuses and gifts from the Babytree company every now and then. Satisfaction!

As I discussed in Chapter Two that Chinese pregnant women and new mothers get routine discrimination in employment and many of them lost their jobs due to childbirth. Babytree gives them an opportunity to achieve success economically. 


\subsection{Conclusion}

This chapter provides insights into the interface design on the app Babytree. The functions on the four main interfaces encourage users to interact with peers and healthcare professionals and build social connections to obtain embodied social support and credible medical expertise. The rhetorical devices used in the regular updates further persuade users to engage with the app, motivating them to follow certain behaviors and evaluate themselves and their children willingly. The interface design increases users' interactivity. Miller (2007) states that interactivity is essential for agency. With the increased biomedical literacy, reliable medical assistance, and sufficient social support, it is likely that users get empowered and reclaim agency in enacting pregnancy and motherhood. The app also includes fathering practice in prenatal education, trying to encourage gender equality in childcare and parenthood, which shows sociocultural changes in the Chinese reproductive discourse. However, the app also constrains users by representing a heterosexual marriage to solidify traditional gendered norms, traditional postpartum practices, and conforming to government regulations such as immunizations for babies. It persuades users to embrace medicalization of reproductive care by encouraging them to do risk management, project management, and self-surveillance. In spite of these constraints, compared with the previous passive role in dominant discourses of reproduction, women can freely make choices in achieving health and good outcomes of pregnancy via the app. In other words, the app provides opportunities for female users to take charge of their body and their health and make informed decisions in reproduction. With their amplified agency, it is possible for them to take action to 
respond to the dominant medical discourse and traditional oppressive Chinese culture in order to achieve good outcomes of pregnancy, childbirth, and parenting. In the next chapter, I delve more deeply into users' posts in the social circles on the app to examine how users take advantage of the digital space to write their embodied personal experience into online narratives to respond to medical dominant discourses and traditional Chinese oppressive culture. I also investigate whether or to what extent users can do transformative practices and promote reproductive justice, and the possibilities of their embodied experience empowering peers. 


\section{References}

Aristotle. Poetics. In W. H. Fyfe (Trans.), Aristotle in 23 Volumes (Vol. 23). Cambridge: Harvard UP.

Rhetoric. In J. H. Freese (Trans.), Aristotle in 23 Volumes (Vol. 22).

Cambridge: Harvard UP.

Baker, L. R. (2011). First-personal aspects of agency. Metaphilosophy, 42(1-2), 1-16.

Biesecker, B. A., \& Lucaites, J. L. (Eds.). (2009). Rhetoric, materiality, \& politics (Vol. 13). Peter Lang.

Burton, G. (2004) Silva Rhetoricae. Retrieved from http://rhetoric.byu.edu/Canons/Style.htm

Campbell, K. S., Riley, K., \& Parker, F. (1990). You-perspective: Insights from speech act theory. Journal of Technical Writing and Communication, 20(2), 189-199.

Carnegie, T. A. (2009). Interface as exordium: The rhetoric of interactivity. Computers and Composition, 26(3), 164-173.

Cicero. (1960). De Inventione. (H. M. Hubbell, Trans.) Cambridge, MA: Harvard University Press.

Dilger, B. (2010). Beyond star flashes: The elements of Web 2.0 style. Computers and Composition, 27(1), 15-26.

Doshi, M. J. (2018). Barbies, goddesses, and entrepreneurs: Discourses of gendered digital embodiment in women's health apps. Women's Studies in Communication, 41(2), 183-203. 
Eyman, D. (2015). Digital rhetoric: Theory, method, practice (p. 177). University of Michigan Press.

Frazier, M. (April 2, 2012) The Data-Driven Paren. The Atlantic.

Hopkins, M. F., and Perkins, L., (1981) Second Person Point of View in Narrative. Critical Survey of Short Fiction. Ed. Frank M. Magill. New Jersey: Salem. 119132.

Hu, Y., \& Scott, J. (2016). Family and gender values in China: Generational, geographic, and gender differences. Journal of Family Issues, 37(9), 1267-1293.

Johnson, S. A. (2014). "Maternal devices", social media and the self-management of pregnancy, mothering and child health. Societies, 4(2), 330-350.

Lakoff, G., \& Johnson, M. (1980). Metaphors we live by. University of Chicago press.

Lanham, R. A. (2006). The economics of attention: Style and substance in the age of information. University of Chicago Press.

Lesikar, R. V. (1990). Business communication (No. 651.74 L5653b Ej. 1 019765). RICHARD D. IRWIN.

$\mathrm{Li}, \mathrm{X}$. (2018). Chinese fathers in the twentieth century: Changing roles as parents and as men. NORA-Nordic Journal of Feminist and Gender Research, 26(4), 331-350.

Long, E. (2009). Metaphor, personification and anthropomorphization in contemporary popular media representations of science. Pacifica Graduate Institute.

Longhurst, R. (1999). Pregnant bodies, public scrutiny: 'Giving' advice to pregnant women. In Embodied geographies: Spaces, Bodies and Rites of Passage. Ed by Elizabeth, K. Routledge: London, UK. 
Lupton, D. A. (2011). 'The best thing for the baby': Mothers' concepts and experiences related to promoting their infants' health and development. Health, risk \& society, 13(7-8), 637-651.

Lupton, D., \& Barclay, L. (1997). Constructing fatherhood: Discourses and experiences. Sage Publications, Inc.

Lupton, D., \& Thomas, G. M. (2015). Playing pregnancy: the ludification and gamification of expectant motherhood in smartphone apps. M/C Journal, 18(5).

Margolin, U. (1990). "Narrative 'You' Revisited”. Language and Style. 23(4): 425-446.

McMillan, S. J. (2002). Exploring models of interactivity from multiple research traditions: Users, documents, and systems. Handbook of new media, 2, 205-229.

Miller, C. R. 2007. "What Can Automation Tell Us About Agency?” Rhetoric Society Quarterly 37(2): 137-137.

Prince, G. (1987) A Dictionary of Narratology. Lincoln: University of Nebraska Press. Quintilian, Institutio Oratoria, in The Orator's Education, 5. vols, Donald A. Russell (trans.), Loeb Classical Library, Cambridge, Massachusetts and London: Harvard University Press, 2001.

Root, R., \& Browner, C. (2001). Practices of the pregnant self: Compliance with and resistance to prenatal norms. Culture, Medicine and Psychiatry, 25(2), 195-223.

Sanders, R. (2017). Self-tracking in the digital era: Biopower, patriarchy, and the new biometric body projects. Body \& Society, 23(1), 36-63.

Wardrip-Fruin, N., \& Montfort, N. (Eds.). (2003). The New Media Reader. MIT press. 
Wen, S., (Febrary, 2, 2012). For All Your Parenting Questions, Babytree.com Got the Answers. Retrieve from http://finance.sina.com.cn/leadership/mroll/20120202/164311300571.shtml

Statistics on the development of health services in 2019. (Wang, H, trans) Retrieved from http://www.nhc.gov.cn/guihuaxxs/s10748/202006/ebfe31f24cc145b198dd730603 ec4442.shtml 


\section{Using digital space to reclaim rhetorical agency}

So far, I have analyzed how the app's interfaces rhetorically position users to build social connections and what rhetorical devices or strategies are used in regular updates to engage users. However, the app's female users are not passive technology consumers; instead, they are savvy users and even producers when engaging with the app. In this chapter, I delve deeply into users' posts on the app to examine how female users take advantage of the technological affordances to write their embodied experience into online narratives and the possibilities of making meaningful changes and empowering other users.

Building on the scholarship claiming that rhetorical agency is not individual autonomous possession, or rising from a static network, rather, it is protean and emerges from dynamic material and rhetorical conditions (Campbell, 2005; Cooper, 2011; Geisler,2004; Hellenbeck, 2016), I argue that rhetorical agency is a kind of capability of critically engaging with ever-shifting material and rhetorical conditions to seek meaningful changes and promote social/reproductive justice and digital technologies as part of the conditions can facilitate rhetorical agency. In chapters four and five, I introduce two examples to demonstrate how female users critically and rhetorically interact with digital technologies such as social media and apps to negotiate, resist, compromise with, and combat dominant discourses of medicine and traditional Chinese culture to promote reproductive justice. I argue that these women are technofeminists and their acts may empower peer users to make changes, too. 
In the following part, firstly, I introduce the research questions, then analyze the ways that these women use language and the strategies in their writing on the app, how they understand and handle childbirth and mothering, and the possibilities to assert rhetorical agency and promote reproductive justice.

\subsection{Research questions}

Since social media have the potential to engage issues of reproductive injustice, I pose the following questions to guide my investigation of how Chinese women use the app Babytree to assert their rhetorical agency and advocate reproductive justice:

1) How did the users engage with the app by writing their embodied pregnant and mothering experience into online narratives? In what ways did they use language and rhetorical strategies in writing?

2) How did they understand and deal with childbirth and mothering?

3) Are there possibilities that they assert their rhetorical agency and promote reproductive justice? If so, to what extent?

\subsection{Methods and Data Coding}

My original design of this research was to interview some users to see whether their agency emerged when interacting with peers and healthcare providers on the app, but because the Chinese government imposes strict censorship on social media, my message function was blocked when I was interacting with users. Then I turned to users' social circles to investigate their posts to see how they take advantage of the app's 
technological affordances to reclaim rhetorical agency and combat reproductive injustice and how their writing may empower peers. The data used here was drawn from 12 users' discussion circles which were established based on pregnant women's expected due date in the year in which I collected this data [my institutions IRB specified that I should not disclose the exact year during which data was collected as a privacy protection]. Because most of the users adopt their usernames or pseudonyms, their age, education, and economic status do not show on the app and they are not considered in this research. I selected 50 posts from 898 posts in those 12 discussion circles in order to cover the full spectrum of the writers' attitudes, their reflections and evaluation of their experiences, and their writing purposes. The posts cover the narratives of both first and second births because I want to examine how experiential knowledge empowers women in childbirth and parenting.

Based on the research questions, I set up criteria for data collection. When collecting data, I examined the tone, the diction, the purpose, and rhetorical strategies. The tone choice and the diction used in the posts are the features of the narratives and the stories that I focus on because I want to understand not only the ways that the narratives and stories were written but also how these women look at their experiences of childbirth and mothering. In addition, the writing of these narratives and stories should be informing, suggesting, complaining or marketing something because I believe that they can reflect the authors' rhetorical agency by increasing readers' awareness and consciousness of something, or displaying their dissent from, or negotiating with or 
resisting the hegemonic medical discourse, or promoting certain product(s). If a post describes its authors' interaction with hospitals and medical professionals, like women's requests or ethical appeals, saying "no" to particular interventions, and their reflections on their experiences and medical discourse and so on, it will be collected as data, while if a post does not show its author's critical reflection on her childbirth experiences, it will not be qualified data.

As an example, the following post does not qualify for review in this analysis:

In the morning, I had abdominal pain. It's like a menstrual cramp, but I didn't care as the pain was irregular. I lay in bed and didn't want to move because my abdomen ached when I stood up. I didn't eat lunch until I had regular abdominal pain in the afternoon before going to the hospital. I was admitted to the hospital at about 6 o'clock. When I was admitted, my cervix was not open. At 8 o'clock, it was opened to 2 fingers. Then I was moved to the delivery room. After eating some food, I got checked and the cervix was opened to 5 fingers. The baby came out after an hour of labor pain on the operating table. The pain was so much that I will never experience it again.

This post is essentially a childbirth log recording details of the birth experience. The author shows little reflection on labor pain in the last sentence. By contrast, the data used in this article are those posts which "may restore women's (oppressed) voices, enabling them to impose rhetorical agency after the fact, as mothers and as mindful, 
healthy people..... The act of writing encourages, or permits, both catharsis and self-analysis" (Owens, 2015, p.115).

\subsection{Ethics}

This research has obtained the approval of the IRB of Michigan Technological University.

The data used in Chapter Four is the existing data. Specifically, the data is the users' posts from 12 circles on the app Babytree in 2019. The posts are about their experiences of childbirth and mothering. There is no direct interaction between the researcher and the app users. The app is for the public, and any registered user can read the information on the app. All the users use their usernames or pseudonyms and there is no possibility of identifying the users' private information such as their real names, personal contact information, and their occupations. Since their identities are unknown, it is not possible or practical to contact users to obtain informed consent.

Nevertheless, in order to avoid unanticipated opportunities to disclose identifiable information, I take the following measures to further protect their privacy:

- Original posts are in Chinese, and I translate them to English. Because a word-for-word translation is not possible, this translation provides another layer of privacy and makes it difficult to identify the original post;

- Whenever possible, I summarize the posts' main ideas or paraphrase the posts in the analysis, minimizing risks of potential identifiable/ sensitive 
information disclosure. In those cases where supporting the analysis requires directly quoting a post, such as when discussing tone, style or writing strategies, the entire post won't be included, nor will the original Chinese be included, only the English translation;

- Additionally, the women's usernames /pseudonyms are not included in the analysis, and the writing does not specify which circle the data is from when analyzing a post.

\subsection{Reasserting Rhetorical Agency on the app Babytree}

\subsubsection{Negotiating and resisting dominant medical practice and discourse}

As discussed previously, the Chinese government enacts policies that medicalize reproductive healthcare and encourage hospital birth. As a result, home birth has been almost completely replaced by hospital birth. In addition, medical professionals and technologies dominate childbirth care. The traditional midwifery care, which provided reproductive experiences and knowledge, has been disregarded and replaced. Pregnant women, especially those first-birth women who have no reproductive experiences, are afraid of labor pain. They are encouraged or even forced to undergo a c-section delivery which is said to be painless, even when they are physically eligible for a natural delivery (Huang, Li, Chen, Wang, \& Zhang, 2004).

In the 50 narratives reviewed herein, 18 narratives describe the authors' childbirth experiences and their reflection upon their delivery experience in the hospitals. The 18 
authors, each of whom gave birth to two children, talk about their c-section delivery or natural birth experience and their interaction with the medical professionals. These women use their personal experience to dispel the misconception that c-section birth is painless and encourage others to negotiate with medical professionals to have a natural birth. The narratives also reveal that their first-birth experience has empowered them in the second childbirth to the point that women resist medical authority. These narratives demonstrate that women regaining rhetorical agency during childbirth is possible and these posts empower both the writer and the readers in asserting their reproductive freedom, especially in the choice of a c-section or a natural birth delivery.

In the 18 posts, the tone is informal by including more interrogatives, exclamations, humor, or sarcasm. The diction is casual with colloquial language, even English words, and popular lines of trendy movies, TV and pop culture. The non-standard Chinese language such as colloquium and slang make the posts easy to read and understand. The 18 authors underwent a c-section in their first birth while 17 of them had a natural delivery in their second birth. One author received a c-section in her second birth due to premature rupture of membranes which could cause amnionitis and fetal infection. They adopt the comparison and contrast rhetorical strategy in writing, comparing their two birth experiences to show that a c-section delivery, which was believed to be painless, causes more pain compared with their natural birth delivery. For example, the author who got two c-section surgeries shared her pain experience as follows: 
Unlike a natural birth delivery, the pain of the c-section delivery begins after the operation. Although almost five years have passed, when I recall the pain, it still makes my tummy numb. The deepest memory is keeping sandbags on my abdomen. Pressing sandbags on the incision made me feel better than death [here the author assumes death is the worst and the pain caused by pressing sandbags on the incision is better than death]. When it's time to keep sandbags on the incision, my family always tried to comfort me by saying that it's almost over and the time was coming to an end. But the pain always lasted very long, not to mention that the sandbags pressed on the incision for a few hours...... After the second c-section delivery, I had a new understanding of pain. The abdomen strap, sandbags and pressing my tummy made me almost unconscious. The most terrifying thing was that at regular intervals, the beautiful nurse came to me and untied the belly strap with her slender fingers, and then pressed my tummy forcefully. Although the pain was unbearable, it was unavoidable because she helped me eliminate the lochia and make the uterine contract, so I had to bear the pain. The contraction pain after the surgery was also terrible, and the pain made me forget to cry [in the original post, "cry" is in English]. Do you think contractions just happen before birth? Do you think that contractions are just for a natural birth delivery? Then, you are wrong!!! On the first night after the c-section surgery, I endured both the pain from the nurse pressing my tummy and the contraction pain. In addition, there are a lot of other memories 
about the pain of c-section delivery. For example, I could only speak when I lay down, but I couldn't speak when sitting or standing due to the incision pain. I had to communicate with people in a whispering tone.

The author uses her personal experience to defy the misconception that c-section delivery is painless; on the contrary, it can bring much pain after the surgery. In the post, the author uses more emotive and personal non-standard Chinese, such as more first-person pronouns, emotional adjectives, interrogatives, and a string of exclamations. This personal language suggests strong emotion and personal opinions about c-section delivery. In the sentence "The most terrifying thing was that at regular intervals, the beautiful nurse came to me and untied the belly strap with her slender fingers, and then pressed my tummy forcefully", the adjectives "slender" and "forcefully" show a little bit of comic sense. The informal tone and casual diction make the post easy to read and understand. The speech-like language can easily engage in interaction, passing on information.

Several Babytree users explicitly suggested that others undergo a natural birth delivery. One of the women wrote,

The (natural birth) delivery was very smooth, the labor was fast, and I didn't get lateral episiotomy. During the labor, I used the breathing method to make the delivery not painful. It's just like I want to poop. This natural birth delivery has cured the trauma that I got in the c-section delivery in my first 
childbirth. Thank me for my persistence and hard work. So, I urge pregnant moms to give a natural birth for your bodies.

Some narratives and stories display that unlike a passive role in the first birth, in the second birth, these women get empowered from their first birth experience and can engage more in the second birth. One author wrote,

My first child was born five years ago. When I went to the restroom in the middle of the night, I found some blood in my underwear. My surprise and joy of being a mother for the first time was very self-evident, and I thought she was really coming. I didn't go to the hospital until the next morning. When the doctor told me it was amniotic fluid, I got stunned. It turns out that the blood was not necessarily blood, and it can be mixed with broken water.... When the broken water was confirmed, the doctor solemnly called a nursing worker who took me to the up-stairs in-patient department in a wheelchair. Without knowing it, I was hospitalized. I even forgot to take pictures to post in my WeChat Moment.

In the first birth, the author was passive. She blindly followed what the doctor told her. During her second childbirth, the author wrote,

Unlike her big sister's continuous crying, the baby girl only cried intermittently. I was lying on the operating table, feeling that this was not right. I was in panic, but I knew that I couldn't interrupt the doctors, which 
would affect them, so I didn't say anything. There was a lot of uncomfortable vomiting sound coming into my ears. I became more worried, what should I do? I didn't care that I was still lying on the operating table and asked the doctors what's wrong with the baby. Fortunately, the doctors were very nice and told me not to worry about the baby. They were cleaning the amniotic fluid for the baby.

Here the author used her previous experience to evaluate her second newborn baby who may have some troubles and expressed her concern for the baby. She wrote, "compared with the first baby, I fed the second baby more adeptly. During giving birth to the first baby, I knew nothing, while during the second one, I was very scared and worried about the baby." The embodied experience empowers her and makes her more rhetorically engaged in the second birth.

In addition to more engagement in the second birth, some women exercised their rhetorical agency by negotiating with or even resisting medical authority. For example, 17 authors wrote that they asked for a natural delivery in the second birth. One of the authors said that she requested a natural delivery in the local hospital, but the doctor insisted on a c-section. She rejected it and traveled to a large city hospital to give birth to her second child. Because her uterus was scarred in the c-section delivery in her first birth, the doctor evaluated her scarred uterus and told her that she could try a natural birth first, though the scar was very thin $(1.3 \mathrm{~mm}$, risky). If things went wrong, they could do csection surgery for her. The doctor asked her to make a final decision whether she'd like 
to have a natural birth delivery. She said, "I was both happy and anxious. I was happy because I could have a natural birth, but I was also worried about unexpected bad fortune". Ultimately, she chose to deliver naturally and successfully gave birth to her second baby under the guidance of medical workers in the delivery room. At the end of the post, she wrote,

Today, I'm writing this article for those moms who had a c-section delivery in the first birth and want a natural birth delivery in the second birth. First of all, you should be physically eligible for a natural birth, then follow the doctor's advice. Second, you should strengthen your confidence in giving birth naturally, so that you can go through the entire labor. In a natural birth delivery, the pain happens before birth. You will be able to tolerate the pain that will be gone soon after birth, and your body will recover quickly. A csection delivery is simply neat during birth. After the surgery, the incision is very painful when walking, and your body recovers slowly.

In their second birth, most of the women successfully negotiated with their doctors for a natural birth delivery, a reflection of an increase in reproductive freedom. Because they were empowered by their previous experience, some women even resisted medical authority in their second birth. One author wrote that before she gave birth, her doctor asked her to stay in hospital when she was in her 39th week of pregnancy, but she thought that there were not any signs of labor, so she stayed at home and did not go to the hospital until her contractions began. During preparation for a c-section delivery, her 
nurse applied a lot of lubricant which made her feel uncomfortable. When the nurse left, she went to the restroom to wash it away. She wrote, "In hindsight, this is definitely the wisest decision I made at that time." These descriptions demonstrate that she was not completely passive as she was in her first delivery. Her rejection of the doctor's advice regarding hospitalization and washing away lubricant indicates her resistance to the medical authority.

These 18 narratives demonstrate that it is important to increase women's reproductive experiential knowledge not only to better inform the mothers about their body, pregnancy, and childbirth, but also to empower them to make decisions for themselves during childbirth. By social media powerful networking, these individual empowering narratives can provide more women with childbirth-related knowledge and educate them, transforming the way they understand and practice childbirth, a crucial step in gaining rhetorical agency in childbirth and obtaining collective reproductive freedom.

\subsubsection{Fighting and compromising with the traditional culture}

Son preference has been prevalent for more than 2000 years in China (Jin et al. 2007). Because of China's strict Family Planning policies since the 1970s, the chance of couples having a son has been reduced. Many people turned to sex-selection technologies for securing a male offspring, which led to the imbalance of sex ratio at birth (SRB) in China (Chu,2001). Although the Chinese government has taken measures to address the SRB imbalance, the cultural preference for a son still impacts decision making (Feng, et al. 2012; Ji, et al. 2015). Many families, especially those whose first child is a girl, 
strongly prefer a boy in the second birth, and many women are forced to have a sexselection abortion. Additionally, traditional postpartum practice remains prevalent in China (Wang, 2019). The strict dietary and behavioral restrictions during the postpartum practice, which were believed to help Chinese women restore physical health, regulate and control Chinese women's bodies and actions. In these 50 posts, there are 11 posts whose authors explicitly reject the feudal notion of son preference and violate the dietary and behavioral rules during the traditional postpartum practices.

The 11 authors narrate their experiences in fighting against the traditional patriarchal norms with a complaining tone and casual diction. Although their families looked forward to a son, these women still decided to give birth to their girl. This decision upset their husbands and their mothers-in-law who are the gatekeepers of the patriarchal mechanism. One author wrote,

My first kid is a daughter, I wanna a second one whether it is a boy or a girl, but my mother-in-law said if it's a boy, I should keep it, but if it's a girl, she let me have an abortion. I didn't want to listen to her, I said that I want my daughter have company no matter it's a boy or a girl, so I didn't go to the hospital for sex identification.

The author used casual diction throughout the post with no punctuation and ungrammatical sentences (I intentionally translated the post into English in correct grammatical sentences and punctuation in order to make readers clearly understand the 
post). The author said that she came from a rural area where son preference remains strong. Nevertheless, she counteracted her mother-in-law's son preference imposition by refusing to get a sex identification testing.

In another narrative titled "Girls are good, why don't people like girls?", the author did the same. She wrote,

Today, I went to the hospital for a four-dimensional examination. Out of curiosity, I asked the doctor who did an ultrasound scan for me to see if it was a boy or a girl. The doctor said it's a girl. My parents and I were particularly happy because we all think that girls are emotionally closer to parents. When I told my husband it's a girl, he was very disappointed, and I dare not tell my mother-in-law it's a girl. I was very unhappy.

The disappointment of the author's husband and mother-in-law about the baby's sex led the author challenge son preference and gender discrimination in the post title. Some women wrestled with contradictory attitudes toward son preference. They wanted to have a boy in the second birth, but when the baby turned out to be a girl, the mother shifted emphasis and prioritized their baby's health over its sex. One author wrote,

I was pregnant with the second child by accident. Because the first baby was still young, I hadn't planned to have a second baby. I went to the hospital for a sex-identification testing. The doctor said it's a boy and I decided to give birth to it. Later, I went to another hospital to do ultrasound scanning and that 
doctor also said it's a boy. But today I went to do checkups again, and the doctor said it's a girl. My first kid is a girl, who is 9 months old in two weeks. I thought the second child was a boy, but it turned out to be a girl. It doesn't matter whether it's a boy or a girl. The most important thing is that they are healthy. The older baby is named Tiantian (sweet), and the second child will be named Mimi (honey).

Whether these women want to have a second child as their first child's company, or prefer a girl with pleasing traits, or pay more attention to a baby's health than its sex, their actions subvert gender stereotypes and traditional Chinese patriarchal norms that restrict reproductive freedom.

These narratives also show how these women challenged the traditional Chinese cultural taboos during pregnancy and postpartum practices, especially the dietary and behavioral restrictions. One author said in her post titled "Daily life during pregnancy", "Today I stole a package of Haagen-Dazs and three pieces of Ferrero chocolate from the refrigerator. My husband would be mad if he knew it. I'm going to put the empty ice cream box back in the refrigerator after eating it". The tone of the narrative is funny. The word "stole" and the sentence "My husband would be mad if he knew about it" indicate that she was not allowed to eat ice cream which is prohibited food for a pregnant woman. However, the title "Daily life during pregnancy" shows that she probably violated the dietary rules often. 
In another post, the author wrote, “Today I cooked Luo Si Fen. But I couldn’t eat too much, I just had one bite of it. The rest of it was eaten by my husband. When he ate it, my mouth was watery." Luo Si Fen is a kind of spicy instant noodles that pregnant women are discouraged from eating. The author tried to follow the dietary rules but failed due to one bite of the restricted food.

Some women did not follow the diet rules during postpartum practices. Usually, in postpartum practices, women are encouraged to eat "hot" foods and avoid "cold" foods. Hot or cold foods classification depends on an intrinsic quality of the food, rather than its temperature (Chou, 2017). Hot foods are usually high in protein, like fish, chicken, eggs, and milk that are believed to help with postpartum recovery and improve breast milk supply but easily make women gain weight. One of the posts wrote that she followed the menus from her best girlfriend during her postpartum period. "My recovery was fast and I didn't gain weight. I didn't eat chicken soup or pig trotter soup all day as my mother-in-law did. Thanks to my best friend." Nowadays, Chinese women pay more attention to scientific knowledge about their diet and behaviors while participating in postpartum practices. In a post, the author described breaking the behavior rules,

It's so hot, I wiped myself with warm water several times a day, but it's still stuffy. I turned on the air conditioning, but my family complained a lot about it. If I don't turn on the air conditioning, I can't bear stuffiness. I tried to reason with my family about how to do postpartum practice scientifically, but they wouldn't listen to me. 
Cold air is believed to easily make people catch cold and sick according to traditional Chinese medicine, especially to women in postpartum practices, who are physically weak due to childbirth. When new mothers are doing postpartum practices, they usually stay in bed with windows closed and no air conditioning is permitted to use. In this post, the author turned on the air conditioning, which apparently violates one of the behavior rules and makes her family unhappy.

The above narratives show that when these women participated in the traditional Chinese practices, they did not completely accept the practices which regulate and control their bodies. They changed some practices and did not obey their family members who played dominant roles in monitoring them during the postpartum period.

These narratives reflect these authors' rhetorical agency in insisting on their right to have a girl baby and breaking dietary and behavioral rules during postpartum practices. Sharing these personal experiences of fighting the patriarchal culture online, such as their experience of rejecting a sex identification test and criticizing and resisting the traditional norm of son preference, will enable more women to defy human rights of violation of girls and gender inequality and discrimination, which is crucial to advocate for reproductive justice.

\subsubsection{Using personal experience as marketing rhetorical strategy}

Chinese women have played a vital role in the economy and the state since they massively participated in social production in Mao's era. But gender discrimination 
endures in employment and workplaces in present day China. Due to childbirth and mothering, many Chinese women lose their jobs. The booming business of social media creates opportunities for such women to make money. On Babytree, for example, post authors earn fees from traffic subsidies (benefits that a post receives for pageviews), advertisement revenue (ads embedded in a user's post will generate revenue when they are clicked by readers), and e-commerce conversion revenue (revenue generated when readers click a product link from the Babytree online shopping platform which is embedded in a post). The Chinese women, who have used Babytree in this way, have "sold" their embodied experiences in reproduction.

Owens (2015) argues in Writing Childbirth that online narratives and stories can "function both as personal writing and as political writing" (p.116). This article argues that, apart from social and political significance, writing and sharing childbirth and mothering narratives and stories can have economic significance, too. In the 50 narratives and stories reviewed, 21 posts adopted rhetorical strategies, such as warnings, Q\& A, examples, anecdotes, comparison and contrast, tips, etc., to attract readers whose reading and purchase will bring economic benefits. One post, under the title of "Stop feeding your baby! Your baby has eaten too much, but you don't know yet!", used the rhetorical strategy of Q \& A to inform new moms of feeding their baby moderately. In the post, the author used standard Chinese and formal diction. The author wrote as follows,

Since being a mother for the first time, many mothers are worried that their babies will not be able to eat enough, so when their children cry, they rush to 
feed them. When the children suck their mouths, they hurriedly feed them. When their children are disobedient, they also quickly breastfeed their children. As a matter of fact, your baby is not that hungry. Your overfeeding may cause unexpected health problems for your baby.

This warning title and the paragraph are written to catch readers' attention. The author then posed three questions, highlighted in bold as follows:

\section{Do the baby's behaviors indicate that he is hungry?}

\section{How to know whether the baby has eaten too much?}

\section{How to feed your baby moderately?}

The Q \&A rhetorical strategy and the layout make the post concise, readable, and informative. The post received 582,000 pageviews generating substantial subsidies for the author.

One post used the comparison \& contrast strategy to promote a device for allergy checkups. The tone of the post is persuasive and the diction is formal. The post exceeds 1,000 words. The author began by describing her first child's allergic treatment in hospitals. When she had a second child, she did not want her son to suffer from allergies again.

Now we have a second child. Because allergies may be inherited, I am worried that my second child will also suffer from allergies. Having learned 
lessons from our first child's allergic experience, my husband and I decided to test our second baby's allergic risk to know allergens in advance and prevent it from happening. Genetic testing can specifically detect whether allergy-related genes are normal. It can predict what my child will be allergic to and thus he can avoid suffering from allergies. He is very young and it is troublesome for us to take him to the hospital, so I plan to do genetic testing for my baby on my own because genetic testing only needs to collect the saliva samples in the mouth. You can do it at home without blood draw, which is convenient and cheap.

This paragraph may attract the attention of readers concerned that their own children will get allergies. The author uses the example of her own child to promote a device for collecting saliva for genetic testing. First, she added screenshots to show how to buy the device online and the available perks. 


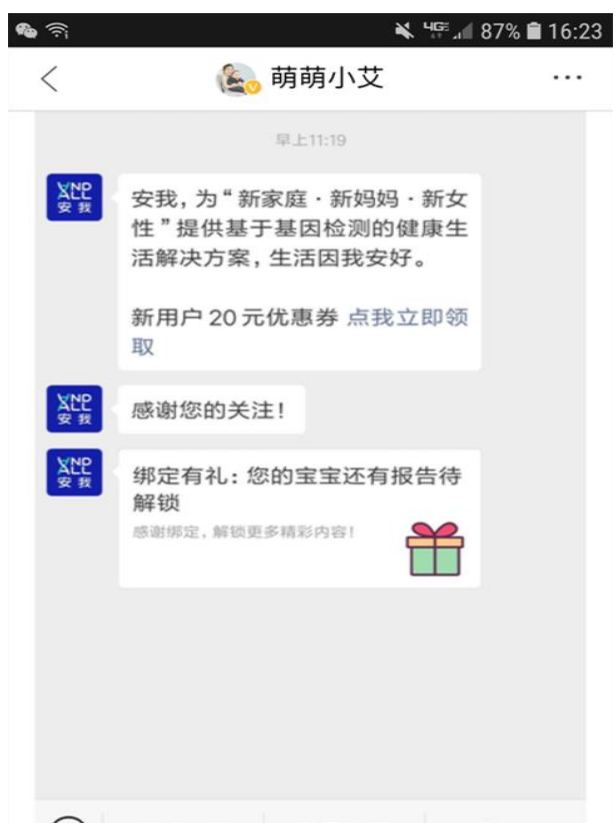

Figure 5: screenshot showing how to buy the device and the perks

Second, she included pictures of collecting her son's saliva to show that the device is easy to operate. 

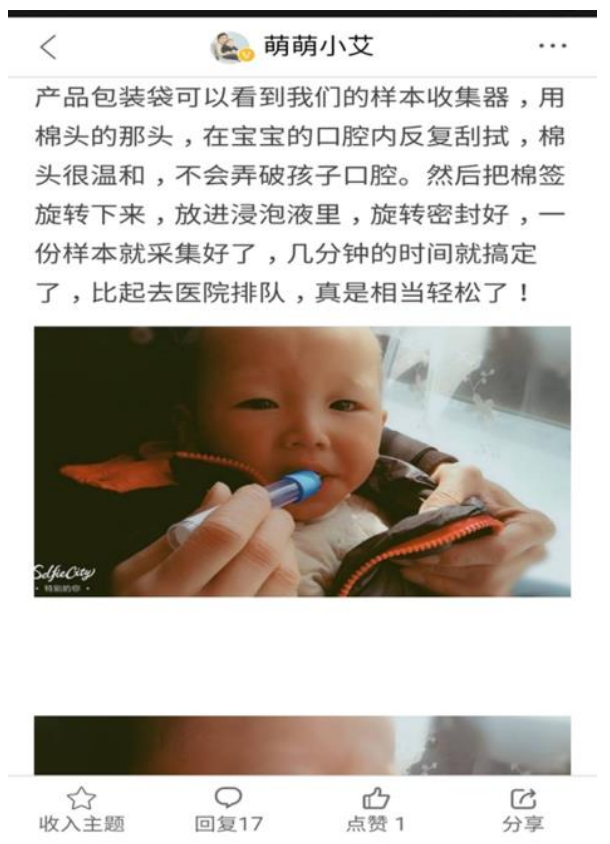

Figure 6: screenshot showing how to collect saliva

She added a screenshot of how to mail the sample and obtain the result.

丰就上门取件了, 运费到付就可以, 老省事

了。

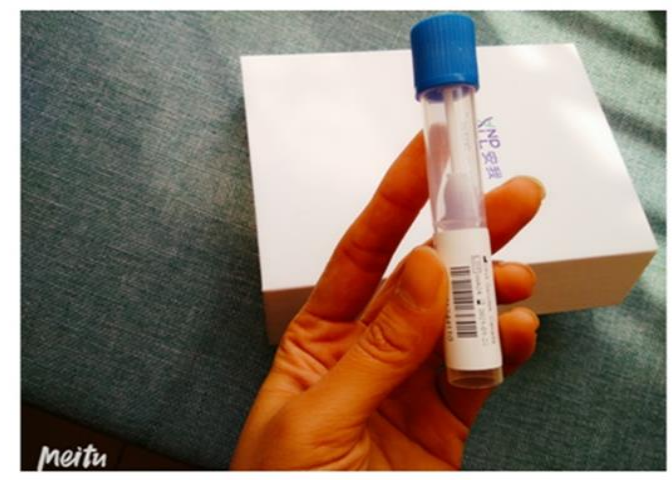

\begin{tabular}{|c|c|c|}
\hline 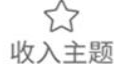 & $\underset{\text { 回复17 }}{Q}$ & $\begin{array}{c}\text { 牟 } \\
\text { 赞 } 1\end{array}$ \\
\hline
\end{tabular}


Figure 7: screenshot showing how to mail the sample

In order to establish credibility of the device, she referenced several authoritative institutions' names including CLIA and CAP. The author compared her two children's experiences to demonstrate that doing allergy check-ups at the hospital is scary and miserable while collecting a child's saliva at home is much easier and relaxing.

This personal mothering story is an effective strategy to persuade readers to buy the device. It garnered 26,000 page views that can bring subsidies to the author. When other users buy this product, the author will receive additional revenue due to the ecommerce conversion. She shared not only her mothering experience but also her usability experience which may make her become a technical communicator in medical and healthcare communication.

Because pregnant women and new mothers are routinely discriminated against in employment, many lost their jobs due to pregnancy and mothering. In order to extend their work, these Chinese women reorient themselves by "selling" their mothering experiences to increase their income. Reproductive justice in this context is closely tied to economic opportunities.

\subsection{Conclusion}

Since China joined the World Trade Organization in 2001, the neoliberal logic which "disseminates the model of the market to all domains and activities" has been prevailing across the country (Brown, 2015). With the digital mundane of smartphones 
and apps, a social media business model is being encouraged and popularizing in China. The Chinese women have identified the intersection of the neoliberal logic and the new business model and caught the opportunity, a "kairos" that "allow subjects to act to change or to reproduce social, institutional, and discursive practices" (Herndl and Licona, 2007, p.134). They take advantage of the technical affordances of Babytree to share their embodied experiences to respond to the traditional norms of motherhood and healthcare provision, and even participate in the social media business to re-enter job markets by "selling" their embodied experiences. These practices reflect that they are savvy technology users as well as bring political and economic transformation. Their acts also emphasize a powerful gender orientation that provides a firm ground for approaching the anxieties of contemporary pregnant women and motherhood in China (article.zhaopin.com). These women's practices are technofeminist and rhetorical acts which promote reproductive justice over time and space by the spreading of social media. In the next chapter, I focus on a case study to examine how a new Chinese mother Mao Wan with postpartum depression used the craft of crocheting to identify with other depressed young mothers on social media and how they worked together to use their crocheted artifacts to do a large-scale installation to raise the public's awareness to seek changes to the dominant biased cultural discourse in China. They became online activists promoting reproductive injustice. 


\section{References}

Campbell, K.K. 2005. “Agency: Promiscuous and Protean.” Communication and Critical/Cultural Studies. 2(1):1-19.

Chu, J. (2001). Prenatal Sex Determination and Sex-selective Abortion in Rural Central China. Population and Development Review 27: 259-281.

Chou, C. (2017). “Traditional postpartum practices and rituals: a qualitative systematic review” (2007), by Cindy-Lee Dennis, Kenneth Fung, Sophie Grigoriadis, Gail Erlick Robinson, Sarah Romans and Lori Ross. Embryo Project Encyclopedia. Retrieved from https://embryo.asu.edu/pages/traditional-postpartum-practicesand-rituals-qualitativesystematic-review-2007-cindy-lee

Cooper, M. M. (2011). Rhetorical agency as emergent and enacted. College Composition and Communication, 420-449.

Feng, X., Xu. L., Guo. Y., Ronsmans, C. (2012) Factors Influencing Rising Caesarean Section Rates in China between 1988 and 2008. Bulletin of the World Health Organization. 90(1), 30-39.

Geisler, C. 2004. "How Ought We to Understand the Concept of Rhetorical Agency? Report from the ARS." Rhetoric Society Quarterly 34(3):9-17.

Hallenbeck, S. (2016) Claiming the Bicycle: Women, Rhetoric, and Technology in Nineteenth-Century America. Carbondal: Southern Illinois University Press. 
Herndl, C.G., and Licona, A. C. (2007). Shifting Agency: Agency, Kairos, and the Possibilities for Social Action. Communicative Practices in Workplaces and the Professions. Ed. Mark Zachry and Charlotte Thraills. Amityville, New York: Baywood, p.133-153.

Huang, P., Li, G., Chen, W., Wang, G., \& Zhang, Y. (2004) The Changing Rate and Indications of Caesarean Section Over 12 Years (H.Wang, Trans.). Academic Journal Guangzhou Medicine College,32:57-99.

Ji, H., Jiang, H., Yang, L., Qian, X., Tang, S. (2015) Factors Contributing to the Rapid Rise of Caesarean Section: A Prospective Study of Primiparous Chinese Women in Shanghai, BMJ Open, 5(11):1-9

Jin, X., Li, S., Feldman, M.W.(2007) Marriage Form and Son Preference in Rural China: An Investigation in Three Counties. Rural Sociology, 72(4), 511-536.

Owens, K.H. (2015). Writing Childbirth: Women's Rhetorical Agency in Labor and Online. Carbondale: Southern Illinois University Press.

Wang, K. (2019). "Reproduction with Chinese characteristics": A Rhetorical-cultural Analysis of the Practice of Postpartum Confinement in Urban China (Master Thesis, University of British Columbia). 


\section{Using material rhetoric to seek changes}

This chapter is focused on a case study. I examine how a new Chinese mother Mao Wan with postpartum depression identified with other depressed moms on social media by the craft of crocheting, and how they work together to do a public crocheting artifact installation as material rhetoric to seek changes, promoting reproductive justice online and offline.

After the overview of my methods and data collection, I provide a description of the frameworks of identification and material rhetoric. Then, I identify and analyze how Mao Wan identified with other depressed moms by the craft of crocheting and sharing her embodied experience and how they worked together to use the crocheted artifacts as material rhetoric in a large-scale art installation to raise the public's awareness and destigmatize postpartum depression. I argue that materiality of embodied experience has potential to intervene in the dominant narratives of mental health in a community such as China with strong stigmatization of mental disorders.

\subsection{Methods and data collection}

The case study in this chapter is focused on a new Chinese mother Mao Wan. She was a 30-year-old mother of two and became an online celebrity in 2019 because of a video clip showing her handrail decorations of a shopping street in the Pudong New Area in Shanghai. Mao suffered mild postpartum depression after she gave birth to her first child in 2015. In order to divert her physical and mental discomfort, she picked up her 
hobby of crocheting, which eventually helped her recover from postpartum depression. She created three crocheting groups on social media totaling about 1,000 members, most of whom were young moms seeking to ease anxiety and pressure by crocheting. With the support of Jinxiu Fang, a property management company in charge of the shopping area, she and other moms from the crocheting groups spent months crocheting cute animals and ornate patterns to decorate the shopping street to mark World Mental Health Day on Oct 10, 2019. Chinese media covered her personal stories about fighting postpartum depression by crocheting. This chapter draws on secondary sources in some influential news outlets in China such as China Daily, The People's Daily, Xinjing Daily, Xinhuanet news portal, Sina news portal, and the Sohu news portal. I collected the following sources related to Mao Wan's online activism and public rhetoric project:

- The interviews of Mao Wan

- The coverages of Mao Wan's stories

- Seven images of her decorating the shopping street

- Ten images of cute crocheted animals sitting on the handrails of the shopping street

- Three images of the handrails decorated with colorful crocheted wipes

- Two images of the signs hang on the handrails

- One images of colorful crocheted umbrellas

- One images of colorful crocheted ice cream 


\subsection{Theoretical Framework}

Kenneth Burke (1969) developed the theory of identification that describes human interaction as more than for persuasion, but also for a process of transformation and change in communication. In A Rhetoric of Motives, he introduces the concept of identification as follows,

"A is not identical with his colleague, B. But insofar as their interests are joined, A is identified with B. Or he may identify himself with B even when their interests are not joined, if he assumes that they are, or is persuaded to believe so. Here are ambiguities of substance. In being identified with $\mathrm{B}, \mathrm{A}$ is "substantially one" with a person other than himself. Yet at the same time he remains unique, and individual locus of motives. Thus, he is both joined and separate, at once a distinct substance and consubstantial with another (1969, p.20-21)".

In other words, A and B are individuals with different lives, different social, political or economic status, but they share interests and come together to work for achieving a common goal, just as he says that "identification is compensatory to division" (p.22). Here Burke's identification sees human interaction more than for persuasion, but also for overcoming division and connecting people, which broadens and complicates the traditional Aristotelean rhetoric of persuasion. Further, Burke indicates that identification is a process of transformation and change in human communication. When a person is identified with another person, he is "both joined and separate, at once a distinct substance and consubstantial with one another" (p.22). He explains that "A doctrine of 
consubstantiality, either explicit or implicit, may be necessary to any way of life. For substance, in the old philosophies, was an act; and a way of life is an acting-together; and in acting together, men have common sensations, concepts, images, ideas, attitudes that make them consubstantial" (p.21). The substances of identification that make two or more participants consubstantial exist in "common sensations, concepts, images, ideas, attitudes". The participants share "the substances" but retain their own individual identity. Besides, "the substances of identification" can induce ways of "acting together", and during the process of identification through consubsantiality, transformation and change occur among the participants. As Shannon Walters (2014) states, "[t]his "actingtogether", similar to Aristotle's “coming into being," brings bodies together in a process of change and alternation" (p.40). Here we can see that Burke's identification focuses more on the social perspective- collective actions.

Burke's identification practice occurs verbally as he sees language as symbolic action to define the notion of rhetorical identification. He states that "you persuade a man only insofar as you can talk his language by speech, gesture, tonality, order, images, attitude, idea, identifying your ways with his" (1969, p. 55). But he also says that "nonverbal, situational factors" can participate in effective persuasion (p.65). Hence, he suggests that rhetoricians do "the analysis of non-verbal factors wholly extrinsic to the rhetorical expression considered purely as a verbal structure" (p.62). Put another way, Burke's identification further extends the traditional Aristotle's (verbal) rhetoric by “exploring the potential of rhetoric beyond verbal means...", as Walters (2014) claims 
that "nonverbal and tactile elements are integral to his movement from traditional rhetorics of persuasion to rhetorics based on identification" (p. 39).

In this chapter, I blend this notion of identification with materiality and material rhetoric as they are used in the field of the rhetoric of health and medicine, such as in Maria Novotny's (2019) work where she and her collaborator Elizabeth Walker help women to create visual artifacts with narratives to express their embodied infertile experience. Those visual stories implicitly serve as material rhetoric that may not only communicate painful experiences to viewers, making them identify with those who want to share the same stories, but also speak back the dominant cultural narrative that assumes a female body is a fertile body and correlates womanhood with motherhood.

Burke's identification does not fully enable an understanding of the necessity and the power of Mao Wan's story. The material dimension moves rhetorical theory (Burke's identification) into a new area that affords researchers better ways of understanding how people can shift their identities. In this case, it allows researchers to understand the impact of Wan's story and the intercultural view of shifting stigma of postpartum depression. The definition of "material rhetoric" was put forward by Michael McGee in the 1980s. He states that "the whole of rhetoric is 'material' by measure of humans experiencing it, not by virtue of our ability to continue touching it after it is gone. $\mathrm{He}$ advocates that rhetoric should be viewed as material "because it survives and records the moment of experience" ((qtd in Biesecker \& Lucaites, 2009, p.23). Many rhetorical scholars have followed his idea in the past few decades by recognizing the material 
impacts of rhetorical tactics and gradually moving the concept of material rhetoric toward social justice movements. For example, Marback (1998) interpreted the closed fist in Detroit as powerful material rhetoric, designed to challenge racism. Similarly, Jamie White-Farnham (2013) identified the red and purple hats and costumes worn by members of a Rhode Island chapter as material rhetoric to address the marginalization or invisibility of aging women. These examples powerfully demonstrate how material rhetoric inspires resistance to social norms and brings attention to marginalized groups.

Taken together, Burke's identification and material rhetoric offer a theoretical lens through which to interpret how Mao Wan identified with other new moms with postpartum depression on social media by means of the craft of crocheting and embodied personal experiences as well as how women decided to use their crocheted artifacts as individual statement on postpartum depression to do large-scale art installations, asserting their rhetorical agency and raising the public's awareness. In this sense, the crocheted artifacts serve as material rhetoric that not only presents postpartum depression to the public, but also intervenes in the dominant narratives of postpartum depression in China. The remainder of this chapter explains the rhetorical significance of Mao Wan and her activist project by offering women space to externalize and symbolically recast the very real pain that they are in. 


\subsection{Using the craft of crocheting to identify with depressed mothers}

According to the coverage of her story, Mao Wan's postpartum depression mirrors experiences of other moms with postpartum depression around the globe, yet her experiences were also greatly related to Chinese sociocultural factors. In an interview with her from Xinjing Daily, Mao Wan said that after she gave birth to the first child, she had difficulty accepting the scar from her C-section. The C-section incision pain and breastfeeding pain constantly bothered her. During her pregnancy, her family gave her substantial attention and care, but after childbirth, they shifted their focus to the newborn baby. She could not mentally adapt herself to the significant change in prenatal and postnatal attention. In addition, the traditional Chinese postpartum practices did not empower her to be a new mom. In the month after delivery, she was confined at her mother-in-law's home, following strict dietary and behavioral restrictions. She was not allowed to eat vegetables, for example, or to open the windows or to turn on the air conditioning, though the weather was stuffy. She had not washed her hair in a month, which made her feel dirty and gloomy; she disliked herself. Moreover, she quit her job and stayed at home to take care of her baby, but she struggled in her new role as a mother. She felt useless and lost interest in life. She cried more than usual and couldn't sleep at night. She became reluctant to care for her baby and feared that she would do something harmful to him (Xu, 2019).

Like many other depressed young moms, postpartum depression stigma prevented Mao acknowledging that she was suffering from postpartum depression, and she refused 
to seek therapy. Instead, she picked up her hobby of crocheting to make some artifacts to combat her negative feelings $(\mathrm{Xu}, 2019)$. Thus, the craft of crocheting turned out to be an effective tool to help her cope with postpartum depression. In an interview, she said that when crocheting the artifacts, she concentrated on the crocheting skills and patterns and forgot the negative feelings. Gradually, she could sleep at night and had more energy to take care of her son $(\mathrm{Xu}, 2019)$. Mao found that crocheting was therapeutic and it helped to define goals: initially, to crochet a small hat and a few pairs of shoes for her baby. When she finished the hat, she took a picture of it and posted the picture on social media. Her friends' likes and praise helped to restore her self-confidence. As Mao Wan put it, "I posted the photo of the baby hat on social media, and it got me so much praise from my friends. I felt that I had finally done something valuable" (Shen, 2019). The sense of achievement that the craft of crocheting brought to her, led her to create a social network account to share her achievements. Through crocheting and sharing, she gradually eliminated her negative feelings and constructed an identity as a confident new mother recovered from postpartum depression. She began interacting with her baby more positively by playing and talking with him, as she gradually accepted her new role as a mother (Shen, 2019). Since Mao Wan shared her crocheting skills and artifacts on social media, the craft of crocheting enacted a rhetorical function: it helped her to identify with her many followers who love crocheting and want to learn it and most of whom were stay-at-home moms. She set up three groups on social media to teach them crocheting skills (Moms weave their way out of depression, 2019). Crocheting works as a means of 
identification by which the group members built a gendered virtual community where they constructed their new identities as a traditional technique learner.

\subsection{Sharing embodied experiences to assert their identity}

Mao Wan and her group members became further identified with one other by sharing their personal embodied experiences of reproduction and life. When teaching crocheting skills, Mao Wan told her group members that she had suffered from postpartum depression after childbirth and how the hobby of crocheting helped her recover from it; meanwhile, the others shared their personal experiences about childbirth, parenting, and working. Mao Wan found that there were many mothers with postpartum depression and they either had not realized the illness or had refused to admit it due to the stigma attached to it. In an interview, Mao Wan explained, "I not only share my crocheting skills, but also ask them about their working and living conditions and whether they have talked with their families (about their postpartum depression), and I suggest they go to visit a psychologist for serious symptoms" (Moms weave their way out of depression, 2019). Gradually, some mothers with light and mild postpartum depression symptoms eased their anxiety and depression symptoms by means of crocheting and eventually fought their way out of postpartum depression (He, 2019). This means of sharing embodied experiences become the key identification practice that helped the depressed mothers acknowledge and assert their identity as individuals suffering from postpartum depression in the affirming social media community. This embodied identification practice improves understanding and prompts transformation and change 
among the group members. Moreover, the transformation and change that crocheting and sharing embodied experience brought to the social media community continues because Mao Wan said that she would explore ways to help the young mothers turn their crocheting works into income, alleviating the stay-at-home moms' income pressure while creating a sense of accomplishment (He \& Wang, 2019).

\subsection{Using material rhetoric to seek meaningful changes}

Apart from teaching crocheting in a social media community, Mao Wan did some research on crocheting and postpartum depression online. She found that in western countries many crocheting lovers did large-scale art installations to raise public awareness of depressed patients, empty nest elderly and autistic children. Mao Wan became inspired and wanted to do a similar public installation in China. Her intention was to alert Chinese society and families to the true nature of postpartum depression such that audiences might be moved to give more attention and care to the mothers with postpartum depression, and, thus, help them recover from it. When she shared this destigmatizing idea with her group members, many of them applauded it. They decided to do a large-scale outdoor installation in the Jinxiufang shopping area (Wang \& $\mathrm{He}$, 2019). In thinking about the meaning of the installation, material rhetoric provides $\mathrm{s}$ a fitting interpretative lens to examine the crocheted artifacts as they bring the public's attention to and raise awareness of postpartum depression,

To achieve their advocacy aim, Mao Wan and her group members took advantage of the public installation to express their perspective on postpartum depression. First, they 
used different colors of yarns to crochet 500 meters of colorful artifacts and more than 600 dolls and animals, decorating the iron handrails in the shopping area (Chen, 2019). The 24 images of the crocheted artifacts collected on the news outlets display some cute dolls, bears, angry birds, rabbits, ice creams, monkeys, and umbrellas. All of them were made in seven colors, which is called the rainbow color, indicating that new moms should feel well enough to enjoy a good and happy mood. They also sewed some signs with written words to educate the public and destigmatize postpartum depression. For example, the public installation included a sign with the words that "[w]e hope that our society can better understand postpartum depression and care more about it. Please face postpartum depression correctly and accompany her with love" indicating that postpartum depression is an illness and that society shouldn't stigmatize it and that families should give more attention and care to the depressed moms ( $\mathrm{Fu}, 2019)$. Another two signs, one saying "[r]efuse to get depressed after childbirth and be a rainbow (happy) mom," and the other saying "Let's do crocheting together to make the unhappy mood gone stitch by stitch," seem aimed at educating women readers on how to cope with a depressed mood following childbirth (Chen, 2019; Fu, 2019). These colorful crocheted artifacts considered in the framework of material rhetoric publicly "speak out" on what postpartum depression is and how to cope with it and bring postpartum depression visibility. They function as material activism in a deliberate and important way in that they attempt to change the public's perception of postpartum depression in a concrete way. As such, the colorful crocheted artifacts in the public installation serve as activism and function rhetorically in terms of providing women with an opportunity to mitigate 
stigma against postpartum depression so that women are better able to obtain family support and, when necessary, medical intervention.

Mao Wan's embodied postpartum depression experience helped make the public crocheting installation very successful and more new moms suffering from the illness came to join her (Moms weave their way out of depression, 2019). Mao Wan said in an interview, "Postpartum depression and depression have always been a serious topic. We hope that through this installation and our joint efforts, more and more people can pay attention to this topic, and patients will be able to recover as soon as possible" (wyrubywang, 2019). These strong statements convey political and rhetorical significance. Though she said that she'd never expected that the outdoor installation would become a hot topic in the media, Mao Wan's embodied postpartum depression experiences and the offline outdoor installation invited a wider range of audiences to become aware of postpartum depression (Chen, 2019). Moreover, by means of social media, the subsequent actions that the crocheted artifacts installation induced further the transformation and change which happened beyond Mao Wan's crocheting social media community, moving forward to rhetorically respond to the dominant narratives of postpartum depression (He \& Wang, 2019).

\subsection{Conclusion}

Through the case study of Chinese moms coping with postpartum depression by crocheting and then using crocheted artifacts in a public installation as material rhetoric to assert their rhetorical agency and raise the public's awareness of postpartum 
depression, this chapter suggests that the materiality of personal embodied experience can be recast into material artifacts such that those suffering from mental health concerns can engage with and identify with audiences. When women come together to share their embodied experiences with postpartum depression, they have a better understanding of postpartum depression, and then their agency emerges and decide to make changes --working together to do the symbolic recasting work. This work becomes possible to rhetorically intervene in the dominant biased and oppressive culture stigmatizing mental health in China. This case highlights the role that social media plays in expanding the circulation of the Chinese mothers' embodied experiences and stories of coping with postpartum depression. The large-scale installation used to work as material rhetoric to raise the public's awareness came into being only after these mothers connected and shared their experiences with each other online. This reinforces the idea that these women are technofeminists who take advantage of social media as a tool to promote social (reproductive) justice. 


\section{References}

Burke, K. (1969) A Rhetoric of Motives. Berkeley: University of California Press.

Chen, S. (June 10,2019) Fighting postpartum depression with wool yarns, this 90-yearold mother is awesome! (H. Wang, Trans.). Zhongxiang News. Retrieved from https://baijiahao.baidu.com/s?id=1635949943248421386\&wfr=spider \&for=pc

Fu, Y., (April 20, 2019) The handrails of the streets in Pudong wearing colorful "sweaters", and the story behind it carried in The People's Daily this morning! (H. Wang, Trans.). Retrieved from http://sh.eastday.com/m/20190420/u1ai12439156.html

He, Y., (April 25, 2019) Why did she dress the entire street handrails in the "sweaters" that she crocheted? Young mom Mao Wan hopes to have more space to show the crochetwork from the group of crocheting lovers. (H. Wang, Trans.). Retrieved from https://finance.sina.com.cn/roll/2019-04-25/doc-ihvhiqax4885091.shtml

He, X., Wang M. (October 10, 2019) Dressing sweaters for the streets: Chinese young mothers using crocheting to ease parenting anxiety. (H. Wang, Trans.). Retrieved from http://k.sina.com.cn/article_213815211_0cbe8fab02000pnmq.html

Marback, R. (1998). Detroit and the closed fist: Toward a theory of material rhetoric. Rhetoric Review, 17(1), 74-92. 
Novotny, M. (2019). The ART of Infertility: Finding Friendship \& Healing After Reproductive Loss. Survive \& Thrive: A Journal for Medical Humanities and Narrative as Medicine, 4(1), 19.

Owens, K.H. (2015). Writing Childbirth: Women's Rhetorical Agency in Labor and Online. Carbondale: Southern Illinois University Press.

Shen, K. (October 11, 2019) Women are weaving their way out of postpartum depression. (H. Wang, Trans.). Retrieved from https://www.shine.cn/news/nation/1910113491/

Walters, S. (2014). Rhetorical touch: Disability, identification, haptics. University of South Carolina Press.

Wang, M., He, X., (October 10,2019) Shanghai "new mom" Mao Wan: using wool crocheting to relieve anxiety. (H. Wang, Trans.). Retrieved from https://www.sohu.com/a/346083200_115402

White-Farnham, J. (2013). Changing perceptions, changing conditions: The material rhetoric of the Red Hat Society. Rhetoric Review, 32(4), 473-489.

Wyrubywang, (April 22, 2019). The popularity of the video affects the hearts of netizens. It turns out that there is more than one "new mom" like her. (H. Wang, Trans.). Xinmin Evening News. Retrieved from https://sh.qq.com/a/20190422/001359.htm 
Xu, W. Ed. Chen, S. (April 23, 2019) Mom born in the 1990s "crocheting for handrails" and calling on the society to pay attention to postpartum depression. (H. Wang, Trans.). Retrieved from http://www.bjnews.com.cn/news/2019/04/23/571191.html

ZX Ed. (October 10, 2019) Across China: New moms weave to fight postpartum depression. Retrieved from http://www.xinhuanet.com/english/2019$\underline{10 / 10 / c \_138461317 . h t m}$

Young mother who suffers from postpartum depression crocheted "sweaters" for the handrails in the entire shopping streets. (May 6,2019) (H. Wang, Trans.) The People's Daily.. Retrieved from https://baijiahao.baidu.com/s?id=1632751926133738315\&wfr=spider\&for $=p c$

Moms weave their way out of depression. (October22,2019) China Daily. Retrieved from https://www.chinadaily.com.cn/a/201910/22/WS5dae6ebfa310cf3e35571df8_2.ht $\underline{\mathrm{ml}}$

Postpartum depression. Retrieved from https://www.mayoclinic.org/diseasesconditions/postpartum-depression/symptoms-causes/syc-20376617

\#37-year-old overseas female returnee with a $\mathrm{PhD}$ degree, with her 5-month-old daughter, jumped off the building to death\# (April 28,2020) [Weibo]. (H. Wang, Trans.). Retrieved from 
https://s.weibo.com/weibo?q=\%2337\%E5\%B2\%81\%E6\%B5\%B7\%E5\%BD\%92 \%E5\%A5\%B3\%E5\%8D\%9A\%E5\%A3\%AB\%E5\%B8\%A6\%E7\%9D\%805\%E4 \%B8\%AA\%E6\%9C\%88\%E5\%A5\%B3\%E5\%84\%BF\%E8\%B7\%B3\%E6\%A5\% BC\%E8\%BA\%AB\%E4\%BA\%A1\%23\&Refer=SWeibo_box 


\section{Conclusion}

\subsection{Research summary}

This research focuses on two cases in which Chinese women take advantage of the affordances of the app Babytree and social media to combat institutional and cultural oppression and promote reproductive justice. As the Introduction part discussed, reproductive injustice is systemic, institutional, and even cultural. In China, Chinese pregnant women's reproductive freedom is extremely constrained by medical institutions and traditional Chinese biased culture. Prenatal care is medicalized and pregnancy is treated as a pathological and medicalized event. Public health policy promotes total inhospital births. In the intensive healthcare system equipped with obstetricians, obstetric nurses, doulas, and bio-medical technologies, women are treated as objects and have little agency in reproduction. In addition, traditional Chinese patriarchal and biased culture still lingers in Chinese society to shape women's reproductive behavior. Son preference causes high rates of sex-selective abortion and severe sex ratio imbalance. Traditional Chinese postpartum practices remain prevalent to influence and control women's body and actions. Because women are confined at home with strict dietary and behavior rules, the outmoded postpartum practices partially trigger postpartum depression. Chinese biased culture stigmatizes postpartum depression and the public do not take it seriously, which makes victims' suffering and pain invisible. What's more, pregnant women and new mothers are discriminated against in employment and many of them lost their jobs due to pregnancy and childbirth. In the oppressive reproductive discourse, Chinese 
women's rhetorical agency is severely constrained. Jones (2016) argues that agency and transformation are essential to overcome oppression, and technology design has the potential to amplify users' agency and promote social justice. Utilizing rhetorical analysis with a focus on rhetorical agency as the primary methodology, this research analyzes how digital health technologies such as the app Babytree and social media may empower users and how users take advantage of technological affordances to reclaim rhetorical agency to fight against the dominant discourses of medicine and culture in China.

Informed by the approaches of usability and user experience design and digital rhetoric, firstly, I analyzed how the app's four interfaces rhetorically position users and map their experience for engagement and enable empowerment. I argued that the main four interfaces on the app work as exordium to engage users and increase their interactivity by the modes of multi-directionality, manipulability, and presence. On the sub-interface of "Q\&A", users can not only obtain peer support and consult with healthcare professionals across the country but also have access to all the information on the interface. This many-to-many communication mode increases a high level of interactivity. With peer support resources and professional medical assistance, it is likely that users get empowered and regain autonomy in healthcare and medical decisions. Meanwhile, on the users' profile interface, users can use several modes to compose and share their posts, such as in an audio mode, or a video mode, or a written message mode, or a multimodal one with audio, words, and videos, to engage more readers and generate income. This content-on-demand model can appeal to users who are interested in writing 
and sharing embodied experiences. In addition, the app provides users with several social schemas to obtain efficient peer support and healthcare assistance and build social connections via the "Q \& A" communication mode and social circles building functions. When users engage with various circles, they may experience high levels of interactivity and feel empowered, though their exploration is constrained within predetermined schemas designed on the app.

The app also adopts various kinds of stylistic strategies in the regular updates and function icons with captions to promote users' agency. In the daily and weekly updates, firstly, I analyzed how the app uses second person narratives to encourage users to engage in pregnancy and emphasize their agency in enacting pregnancy, health management, and a family's intimate bonding; then, I investigated how the app employed personification and visuals to make the fetus become a real baby, encouraging users to engage in health responsibility and management and build a virtual family bonding; next, I focused on the function icons with captions on the user's profile interface to examine how the app adopts the rhetorical device of metaphors to compare the prenatal instructions and parenting practices into the tools for completing a project. In other words, pregnancy and parenting is like accomplishing a project, which gives users a concrete framework to understand pregnancy and parenting. This metaphoric strategy can foster users' agency and encourage them to engage with the app to manage health and parenting; then, I analyzed how the app uses the first-person pronoun "I" to ascribe agency to users by investing the icons with captions on the user's profile interface. I 
argued that the first-person pronoun "I" gives users a sense of mastery or control, increasing their awareness of the functions and prompting them to engage with the app willingly; finally, I examined how the app uses bright colors such as pink and yellow to highlight the commercial icons to encourage consumption and entrepreneurship.

Through analysis of the interface design and digital rhetoric on the app, I argued that it is possible that users get empowered and regain agency in pregnancy and parenthood by engaging with the app to increase the biomedical literacy and obtain reliable medical assistance and peer embodied support, though the app, to some extent, conforms to traditional gendered norms, promotes postpartum practices, and aligns with government regulations such as immunizations for babies. This user-generated-content and community-built technology design enables empowerment by encouraging users to engage with the app. Light and Luckin (2008) state that "justice requires us to enable people to engage in the activities necessary to achieve what they want, rather than to give them what they want" (p.9). From the perspective of user experience design, the app provides users opportunities to regain agency by navigating in different social schemas. In other words, through engagement with the app, users may have agency over their body and reproductive healthcare, and even perform transformative practices.

By rhetorically examining users' posts on the app, in Chapter four, I investigated how users take advantage of the app's affordance--- user generated-content nature--- to share their embodied experience to make their voices heard. I argued that some users dispel the misconception of painless c-section, suggest natural birth, negotiate and even 
reject medical authority; some users reject the son preference notion, and some violate the dietary and behavioral regulations during performing the traditional postpartum practices; others use their personal parenting stories as marketing rhetorical strategy to increase income. I argued that with the neoliberal logic and the social media business model in China, these women take advantage of the technological affordances on the app to respond to the dominant healthcare and traditional cultural norms. They actively participate in the app's social media business model to enter job markets by "selling" their embodied experiences, making political and economic changes. Their acts are technofeminist, rhetorical, and transformative practices that may empower peers and promote reproductive justice in the context of the app.

As scholarship has identified that digital technologies such as social media are a potential tool to promote social justice, in Chapter five, in the frameworks of Burke's identification theory and material rhetoric, I analyzed how a Chinese new mother Mao Wan with postpartum depression used the traditional craft of crocheting to identify with other depressed mothers on social media. They shared embodied experience on postpartum depression in the digital space and became further identification with one another. When they worked together, they regained agency and used crocheted artifacts to do a public installation to combat stigma against postpartum depression. The public installation works as material rhetoric to externalize their suffering and pain, which not only raises the public's awareness but also combats the biased culture stigmatizing postpartum depression. 
In addition, this case also echoes the scholarship that social media has the potential to promote reproductive justice. It aids in theorizing how reproductive injustice occurs in relation to institutional and cultural oppression in different cultures and groups, and how digital spaces are an ideal place for women to reclaim rhetorical agency in enacting intercultural communication of reproductive justice.

\subsection{Implications}

\subsubsection{Theoretical implications}

Technofeminism

In this research, one of the theoretical implications is on technofeminism. In the argument of this research, I have tried to demonstrate that not only digital technologies such as social media and apps related to pregnancy and parenthood can impact (empower and constrain) women but women can make use of them to do transformative practices as well. As Wajcman (2004) claims that technologies should not be reduced to the "good" or the "bad". Technologies should be addressed "through use, and use is mediated by rhetoric...... or rhetorical activities" (Hallenbeck, 2015, p. 168). In addition, technologies should "be properly characterized as contingent and open, expressing the networks of social relations in which they are embedded" (Wajcman, 2006, p.108). In other words, technologies are not neutral tools, instead, they are part of social materials and have political and cultural meanings. They shape users and the entire society and at the same time they are shaped by users, especially when they interact with embodied users. In this sense, it is safe to say that digital health technologies such as social media and apps are 
embodied when they influence users' embodied experiences. During the reciprocal embodied shaping, users may experience the digital technologies differently due to their various constructed identities in digital spaces. Thus, the technofeminism approach can better investigate relationships among technologies, users, and power to enable much more democratic and equitable human experience. With this framework, scholarship has examined digital or technological experience of gender and race, and digital identities. It mainly focuses on technofeminist activism to "push back against, upend, and redress these patriarchal — and oftentimes racist, classist, ableist, ageist, homophobic, and other cultural — biases and to reimagine revised and more equitable relationships with technologies and in technology-rich workplaces and living spaces" (Frost \& Haas, 2017, p.90).

Similarly, in this research, Chinese women's rhetorical agency is constrained by the dominant institutional and cultural discourses, they turn to health apps to seek support and assistance. The technicality of digital health technologies such as the app Babytree as a medium provides users with a new pathway to respond to the sociocultural and political situation in the Chinese society. Through the app, users may increase their ability to access maternal and childcare information and support and obtain medical assistance from peers and healthcare professionals. The empowered users may reclaim their authority in terms of understanding their own bodies, and gaining confidence and control over their healthcare, maternal and childcare. Meanwhile, they take advantage of the technological affordances to share their embodied experiences about pregnancy and 
parenthood to support other peers and fight against the dominant institutional and cultural discourses on the app, producing new narratives of reproduction, health, and women's body. These rhetors' speaking back to the dominance discourses may encourage other women who have similar unjust experiences to share their own stories and build alliances. In other words, digital technologies play a substantial role for these women to engage in collective transformative practices. Mao Wan's case is a good activism example about how those depressed mothers from different places in China built coalitions on social media by sharing embodied experiences of postpartum depression. They worked together to combat the biased Chinese culture stigmatizing postpartum depression. They are not passive technology consumers, instead, they are rhetors and technofeminists, taking advantage of digital affordances to advocate activism online to intervene in dominant discourses.

\section{Identification}

Another theoretical implication in this research is Burke's identification theory, which generally focuses on verbal expression. In this research, I focused on non-verbal symbolic means of crocheting used for identification in Mao Wan's case.

Historically, the practice, almost exclusively by women, of crocheting and other textile crafts are tied to traditional views of femininity and can be seen as a result of gender oppression. Nevertheless, these traditional feminine crafts can also be employed in a meaning-making manner with a rhetorical significance (Pristash,2014). For example, 
needlework, quilt making, and crochet are used for donation, fundraising or promoting activist work which is called craftivism (Greer, 2014; Pristash,2014). In Mao Wan's case, she used the craft of crocheting to identify with other depressed mothers on social media. The craft of crocheting brought the individuals together and allowed them to locate themselves in the social media community. The craft of crocheting developed into more than just a hobby. It became a symbolic action. It works as a nonverbal means of identification by which the group members built a gendered virtual community where they constructed their new identity as a traditional technique learner, and at the same time, they retained their individual autonomy in their real life. Burke observes that "[s]ince identification implies division, we found rhetoric involving us in matters of socialization and fiction" rather than limiting rhetoric to more traditional forms of persuasion (Burke, 1969, p.45). Among Mao Wan's group members, the division that Burke mentions is bridged and social connection is formed when the social media community uses the craft of crocheting as nonverbal rhetoric to identify with one another.

When they shared their personal embodied experiences of postpartum depression in the highly gendered virtual community, the process of identification among the group members became complicated. They identified with one another by not only a common interest of the traditional craft of crocheting but also their same embodied personal experiences of suffering from postpartum depression. This means of sharing embodied experience become the key identification practice that helped the depressed moms acknowledge and assert their identity as individuals suffering from postpartum depression 
in the social media community. The embodied identification practice improves understanding and prompts transformation and change among the group members. When they worked together to use their crocheted artifacts to do a large-scale installation to raise the public's awareness, their cooperative feminist practice further extends the process of identification among the community — acting together to make a rhetorical meaning. Burke (1969) states that rhetorical language is inducement to action, while in this case the craft of crocheting as nonverbal rhetoric serves as an inducement to action (p.42). In addition, the place where the process of identification occurred shifts from the virtual space in social media to the physical world, which shows an extension of the identification process.

\subsubsection{Practical implications}

One of the practical implications in this research is online activism. Online activism is not new in China; it first appeared in the late 1990s (Yang, 2009). With the advancement of communication technologies, activists have identified the potential of the Internet, especially social media, as a force for social change (Sutton \& Pollock, 2000; Tai, 2015). Despite the government's sophisticated political control of online space, the past three decades in China have seen more frequent and influential online activities for various marginalized interests (Yang, 2009). For example, grassroots environmental activists use social media as a platform to share information, build social networking, mobilize the public, and construct a green public discourse (Sima, 2011; Sullivan \& Xie, 2009). Because LGBT and feminist activism is strictly censored, social media is an 
important avenue for gender-related grassroot activists to present their voices and put up collective resistance (Cao \& Guo, 2016; Chase, 2012; Hou, 2015; Mao, 2020). In order to achieve the best outcome of their advocacy, feminism activists adopt various online strategies such as further politicizing women's private matters for provoking heated online discussion and building online coalitions to create space for women's voice (Hou, 2020; Wang \& Driscoll, 2019).

Mao Wan's case continues the scholarship on Chinese online activism. Her feminist online activism not only contributes to the Chinese feminist movement, but it also echoes western feminist scholars' work, especially in the field of RHM where breastfeeding women, pregnant teenagers, new moms, and infertile women employ their personal embodied experience or stories as rhetorical interventions to combat dominant social, political, institutional, and cultural discourses (Koerber, 2006; Owens, 2015; Seigel, 2014; Vinson, 2018). This case, thus, also highlights the role that social media plays in expanding the circulation of the Chinese mothers' embodied experiences and stories of coping with postpartum depression. The large-scale installation used to raise the public's awareness came into being only after these moms connected and shared their experiences with each other online. This reinforces the idea that social media is a potential tool to promote social justice. Put another way, this research result has validated that Mao Wan and other activist young mothers succeeded smartly using digital technologies such as social media as a rhetorical tactic to raise the public's attention, effecting social changes. Those oppressed and marginalized Chinese people could follow 
this case and continue taking advantage of social and digital media to enter the public arena in which they can produce new or alternative narratives to intervene in dominant discourses.

Another practical implication of this research is digital rhetorical practice and digital economy. In The Economics of Attention, Richard Lanham (2006) argues that digital rhetoric can facilitate an economic model to increase monetary and social capital by maintaining users' attention. James Porter (2010) states that digital economics is an important part of digital rhetoric and "rhetorical contexts themselves rely on an economic system of exchange... an exchange of value that serves as the motivation for the production and circulation of digital objects" (p.174). He argues that digital rhetoric such as useful information and knowledge can help shape and influence the digital economy as well as social networking. In China, the online economic model, especially social media business model, is getting more and more popular. The app Babytree, which is a commercial app, serves as a good example. In order to attract users' attention and maintain their engagement, the app adopts various rhetorical strategies and functions to facilitate users to create and circulate information and knowledge related to childbirth and parenthood. For example, healthcare professionals can "sell" online classes on pregnancy and motherhood and offer online consulting service and diagnosis by phone or messages. Pregnant women and new mothers can create multimodal posts to share their embodied experience in the circles generating income. The app's interfaces work as digital exordium to potentially increase users' interactivity and match their desired expectations 
and engagement, making them "well-disposed, attentive and receptive" for persuasion and empowerment. Digital rhetorical practitioners in an economic model can get inspiration from the app and develop and design more engaging digital rhetorical strategies and functions to accrue economic value.

The third practical implication of this research is digital health technology design. As most digital health technology design focuses on usability and usefulness, it reduces health technologies to the use of context. As a matter of fact, patients including pregnant women and new mothers often experience a wide range of physical and psychological changes (symptoms). Failure to address these symptoms or changes, especially psychological ones, will lead to worse health conditions such as distress or depression. Patient users need peer support in handling illness or are willing to share their experiences and information with other patients. In addition, health literacy can significantly impact users' use of digital health technologies such as health apps and their application of the knowledge they have obtained to manage their health. How to address these issues and challenges is an important component in design. The app Babytree can serve as inspiration for digital health technology design. It consists of four main interfaces with many sub-interfaces where users can gain peer support, reliable medical professionals' assistance, self-management tools, and readable healthcare information and knowledge. This community-built design empowers users more comprehensively than the function-oriented design does. 


\subsection{Future directions for scholarship}

In this research, I argue that the app Babytree may empower users and provide users the potential to promote reproductive justice. In the future, I will recruit some users to do usability studies to examine whether or to what extent the app's design and functions empower and constrain users. I will interview users to talk about their experience of engaging with the app to see whether they claim agency and have a new understanding of pregnancy and parenthood. Since the app is available for Chinese users in the United States, I would also like to interview some Chinese American users to do cross-cultural communication studies on pregnancy and motherhood. At last, I would suggest that scholars continue to investigate non-western contexts for a fuller picture of how digital technologies do or do not facilitate rhetorical agency in the fields of the rhetoric of health and medicine and technical communication. 


\section{References}

Burke, K. (1969). A rhetoric of motives. Univ of California Press.

Cao, J., \& Guo, L. (2016). Chinese “Tongzhi” community, civil society, and online activism. Communication and the Public, 1(4), 504-508.

Chase, T. (2012). Problems of publicity: Online activism and discussion of same-sex sexuality in South Korea and China. Asian Studies Review, 36(2), 151-170.

Frost, E. A., \& Haas, A. M. (2017). Seeing and knowing the womb: A technofeminist reframing of fetal ultrasound toward a decolonization of our bodies. Computers and Composition, 43, 88-105.

Greer, B. (Ed.). (2014). Craftivism: The art of craft and activism. Arsenal Pulp Press. Hallenbeck, S. (2015). Claiming the bicycle: Women, rhetoric, and technology in nineteenth-century America. SIU Press.

Hou, H. L. (2015). On fire in Weibo: Feminist online activism in China. Economic and Political Weekly, 79-85.

. (2020). Rewriting "the personal is political": young women's digital activism and new feminist politics in China. Inter-Asia Cultural Studies, 21(3), 337-355. 
Jones, N.N., (2016) Narrative Inquiry in Human-Centered Design: Examining Silence and Voice to Promote Social Justice in Design Scenarios. Journal of Technical Writing and Communication. 46(4): 274-492.

Koerber, A. (2006). "Rhetorical agency, resistance, and the disciplinary rhetorics of breastfeeding”. Technical Communication Quarterly, 15, 87-101.

Lanham, R. A. (2006). The economics of attention: Style and substance in the age of information. University of Chicago Press.

Light, A., \& Luckin, R. (2008). Designing for Social Justice: People, Technology, Learning. Bristol, UK: Futurelab.

Mao, C. (2020). Feminist activism via social media in China. Asian Journal of Women's Studies, 26(2), 245-258.

Owens, K.H. (2015). Writing Childbirth: Women's Rhetorical Agency in Labor and Online. Carbondale: Southern Illinois University Press.

Porter, J. E. (2010). Rhetoric in (as) a digital economy. Stuart Selber (Ed.) Rhetorics and technologies: New directions in writing and communication, 173-197. Columbia, SC: University of South Carolina Press.

Pristash, H. E. (2014). A sharper point: a feminist, multimodal heuristic for analyzing knitted rhetoric (Doctoral dissertation, Bowling Green State University). 
Seigel, M. (2014). The Rhetoric of Pregnancy. Chicago: The University of Chicago Press.

Sima, Y. (2011). Grassroots environmental activism and the Internet: Constructing a green public sphere in China. Asian Studies Review, 35(4), 477-497.

Sutton, J., \& Pollock, S. (2000). Online activism for women's rights. CyberPsychology \& Behavior, 3(5), 699-706.

Sullivan, J., \& Xie, L. (2009). Environmental activism, social networks and the internet. The China Quarterly, 422-432.

Tai, Z. (2015). Networked resistance: Digital populism, online activism, and mass dissent in China. Popular Communication, 13(2), 120-131.

Vinson, J. (2018). Embodying the Problem: The Persuasive Power of the Teenage Mother. New Brunswick, Camden, and Newark, New Jersey, and London: Rutgers University Press.

Wajcman, J. (2006). Technocapitalism meets technofeminism: women and technology in a wireless world. Labour \& Industry: a journal of the social and economic relations of work, 16(3), 7-20.

Wang, B., \& Driscoll, C. (2019). Chinese feminists on social media: articulating different voices, building strategic alliances. Continuит, 33(1), 1-15. 
Yang, G. (2009). China since Tiananmen: online activism. Journal of Democracy, 20(3), 33-36. 